\title{
RECOVERY OF INTERBLOCK INFORMATION
}

by

Anis I. Kanjo, M.S.

Thesis submitted to the Graduate Faculty of the Virginia Polytechnic Institute

in candidacy for the degree of

DOCTOR OF PHILOSOPHY

in

Statistics

May 1965

Blacksburg, Virginia 
TABLE OF CONTENTS

Page

1. INTRODUCTION . . . . . . . . . . . . . . 4 4

2. THE CONVENTIONAL METHOD OF COMBINING ESTIMATES

IN INCOMPLETE BLOCK DESIGNS. . . . . . . . . . 7

3. THEOREM 1.................... . 14

3.1 Remarks . . . . . . . . . . . . . 20

3.2 Use of Theorem 1. . . . . . . . . . . 22

4. Lemma 1. . . . . . . . . . . . . . . 24

4.1 Corollary . . . . . . . . . . . . 26

5. LEMMA 2. . . . . . . . . . . . . . 28

5.1 Corollary 1.............. . 30

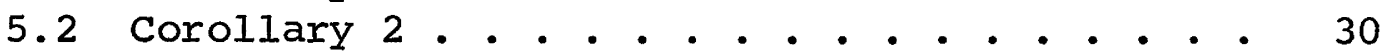

6. SOME RELATIONS IN B.I.B. DESIGNS . . . . . . . 31

7. THEOREM 2. . . . . . . . . . . . . . . 33

7.1 Exact Results for Theorem 2........ 42

7.1.1 Method for computing F........ 44

7.2 Application of Theorem 1 in B.I.B. Designs. . 46

7.3 Procedure for Recovery of Inter-block

Information in B.I.B. Designs . . . . . . . 49

7.4 Worked Example. . . . . . . . . . 51

8. P.B.I.B. WITH TWO ASSOCIATE CLASSES. . . . . . 56

8.1 Definitions and Useful Relations. . . . . . 56

8.2 Variances and Covariances . . . . . . . 59

8.3 Sigh of the Quantity C-C' . . . . . . . . 63

8.4 Theorem 3................ . 65

8.5 Special Case. . . . . . . . . . . . 70

8.6 Recovery of Inter-block Information in

Group Divisible Designs . . . . . . . . . 75

8.6.1 Recovery in Regular Group Divisibles . 76

8.6.1.1 Study of the Ratio

$\left(\mathrm{V}-\mathrm{C} /\left[\mathrm{V}-\mathrm{C}+\mathrm{n}\left(\mathrm{C}-\mathrm{C}^{\prime}\right)\right] . . . . \quad 81\right.$

8.6.1.2 Application of Special Case

of Theorem 3........ 88 
TABLE OF CONTENTS (cont.)

Page

8.6.2 Analysis for Singular and Semi-regular

G.D. Designs . . . . . . . . . .

8.6.2.1 Inter-estimates and Recovery

of Inter-information in

Singular G.D. . . . . . .

8.6.2.2 Inter-estimates and Recovery

of Inter-block Information

in Semi-regular G.D.

8.7 Recovery of Inter-block Information in LS

Type. . . . . . . . . . . . . . 102

8.7.1 Within Comparisons ... . . . . . 110

8.7.2 Among Comparisons. . . . . . . . 112

8.8 Triangular P.B.I.B. Designs . . . . . . . 115

8.8.1 Definition and Comment on the singu-

larity of the Inter-Analysis Model • . 115

8.8.2 Application of Theorem 3 When $\mathrm{V}\left(\mathrm{z}_{i}\right)$

is of the Form V-C+ $\rho\left(\mathrm{C}-\mathrm{C}^{\prime}\right)$. . . . . 119

8.8.3 Recovery of Inter-block Information

in Triangular Designs. . . . . . . 123

8.8.3.1 study of the Ratio

$\left[\mathrm{V}-\mathrm{C}+\rho\left(\mathrm{C}-\mathrm{C}^{\prime}\right)\right] /(\mathrm{V}-\mathrm{C})$. . . . 129

8.8.3.2 Combined Estimates for

Triangular Designs. . . . . 131

8.9 Cyclic P.B.I.B. Designs with Two Associate

Classes . . . . . . . . . . . . . . 132

8.9.1 Definition . . . . . . . . . . . 132

8.9.2 Recovery of Inter-block Information

in Cyclic Designs. . . . . . . . 133

8.9.2.1 Combined Estimates for

Cyclic Designs. . . . . . 143

8.10 General Procedure for Recovery of Inter-

block Information in P.B.I.B. with Two

Associate Classes . . . . . . . . . . 144

8.11 Comment on the Numerical Methods Used in

Table VII Through Table X . . . . . . . 147

9. SUMMARY AND DISCUSSION . . . . . . . . . . . . 151

10. ACKNOWLEDGEMENTS . . . . . . . . . . . . . . 154

11. TABLE OF REFERENCES. • . . . . • • • • • • • • . 155

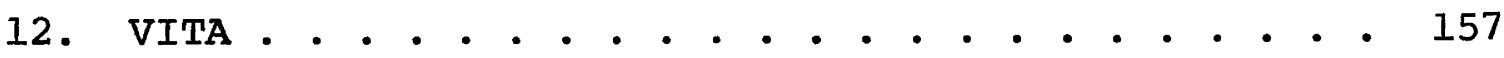

TABLES • • . • . . . . . . . . . . . . . . 158 


\section{INTRODUCTION}

The problem of the recovery of inter-block information in incomplete block designs was recognized by Yates (10) in 1939. The idea is to combine the two independent intra- and inter-block estimates in order to increase the accuracy of our estimation of the treatment effects, and consequently to recover some of the efficiency that was lost by reducing the number of plots in a block from $v$, the number of treatments, to some number $\mathrm{k}<\mathrm{v}$.

The best linear combination of the intra-block and inter-block estimates is:

Intra-variancexInter-estimate+Inter-variancexIntra-estimate Intra-variance + Inter-variance

However this combined estimate is merely theoretical since there is, in practice, no knowledge about the exact interand intra-variances. A reasonable way of overcoming this difficulty is to use a random weight which can be computed from the data of our experiment.

Yates suggests in (10) a rather complicated expression for the combined estimate in the B.I.B. designs. Later in 1960, V. Seshadri (8) has shown that Yates' combined estimate 
is unbiased and that it is uniformly better (better in the sense of less variance) than the intra-block estimator only when $\frac{\sigma_{\mathrm{b}}^{2}}{\sigma^{2}}$ is smaller than $\frac{r}{\lambda v}$. Although no specific results were available about the case when $\frac{\sigma_{\mathrm{b}}^{2}}{\sigma^{2}}>\frac{r}{\lambda v}$, nevertheless Seshadri's work indicates clearly that Yates' combined estimator is not uniformly better than the intra-block estimate when $\frac{\sigma_{\mathrm{b}}^{2}}{\sigma^{2}}>\frac{r}{\lambda v}$.

In (7) Rao, C.R., has established what we shall call the conventional method of combining estimates in any incomplete block design. This method was adopted by Bose, R.C., in (1) and is the method pursued in practice almost everywhere.

Graybill in (4) suggests a combined estimator which is uniformly better than the intra-estimate provided severe restrictions are placed on the size of the experiment.

Seshadri, V., in (9) combines two estimates $x$ and $u$ in the balanced case in such a way that the combined estimate is uniformly better than both $\mathrm{x}$ and $\mathrm{u}$ provided that $\mathrm{v}>5$. It should be noted that he misnames $x$ and $u$ the inter- and intra-treatment estimates, respectively; in fact, his $x$ and $\mathrm{u}$ are special linear functions of the observations as they 
were previously defined in (3) by Graybill and Weeks. This article, however, is suggestive and indicates that something can be done which permits an effective utilization of the idea of the recovery of inter-block information even when $v$ is small.

In general, one can say that there is, so far, no practical solution to the problem without severe restrictions on the size of the experiment, and no solution at all for a clear and precise answer to the question of how much is recovered. In fact, regardless of how large the experiment is, the experimenter applying the methodology available to him now cannot be sure that he is really improving the accuracy of his estimation.

In this dissertation, after giving a brief critique of the conventional method of recovering the inter-block information, a practical solution which avoids the handicaps of the conventional method in B.I.B. designs will be discussed. This practical solution is extended to the P.B.I.B. designs with two associate classes. Finally, an exhaustive enumeration of the amount of recovery achieved or a lower bound of it is given. 
2. THE CONVENTIONAL METHOD OF COMBINING ESTIMATES IN INCOMPLETE BLOCK DESIGNS

The most widely used method is that of Rao (7), where the intra- and inter-normal equations are combined in the same way that two independent estimates are usually combined, that is by first weighing inversely by the variance of each and then obtaining a least squares joint solution. Bose (1) gives the resulting combined intra- and inter-block estimate $\mathrm{T}_{i}$ of treatment $t_{i}$ as

$$
r\left\{w^{\prime}+w(k-1)\right\} T_{i}=\left(k-d_{2}\right) P_{i}+\left(d_{1}-d_{2}\right) s_{1}\left(P_{i}\right),
$$

where

$$
\begin{aligned}
& \mathrm{w}=\frac{1}{\sigma^{2}}, \\
& w^{\prime}=\frac{1}{\sigma^{2}+k \sigma_{b}^{2}}, \\
& P_{i}=w Q_{i}+w^{\prime} Q_{i}^{\prime},
\end{aligned}
$$

$\mathbf{Q}_{\mathbf{i}}$ and $\mathbf{Q}_{\mathbf{i}}^{\prime}$ are the adjusted yields in the intra- and interanalysis respectively,

$$
a_{j}=\frac{c_{j} \Delta+r \lambda_{j} w}{\Delta+r H w+r^{2} w^{2}}, \quad(j=1,2)
$$

and,

$$
W=\frac{W^{\prime}}{w-W^{\prime}}
$$


$\mathrm{r}, \mathrm{k}, \lambda_{1}, \lambda_{2}, \mathrm{c}_{1}, \mathrm{c}_{2}, \Delta$, and $\mathrm{H}$ are defined in reference (1), and they will be defined explicitly in the sequel.

In practice, estimates for $\sigma^{2}, \sigma_{b}^{2}$ are usually obtained from the analysis of variance table, namely:

$$
\hat{\sigma}^{2}=s_{e}^{2}
$$

and

$$
\hat{\sigma}_{b}^{2}=\frac{b-1}{N-v}\left(S_{b}^{2}-S_{e}^{2}\right)
$$

where $\mathrm{S}_{\mathrm{e}}^{2}$ is the error mean square and $\mathrm{S}_{\mathrm{b}}^{2}$ is the blocks adjusted mean square. Since $\hat{\sigma}^{2}$ and $\hat{\sigma}_{b}^{2}$ obtained from the analysis of variance table are consistent estimates for $\sigma^{2}$ and $\sigma_{\mathrm{b}}^{2}$, these estimates would be good enough to represent the unknown parameters $\sigma^{2}$ and $\sigma_{b}^{2}$ if estimated from a large experiment.

The questions now arise: (a) How reliable is the substitution of these estimates into a complicated expression as (1)? (b) If $\hat{\sigma}$ and $\hat{\sigma}_{b}^{2}$ are estimated from a large experiment, do the estimates $\hat{w}=\frac{1}{\widehat{\sigma}^{2}}$ and $\hat{w}^{\prime}=\frac{1}{\frac{\pi}{\sigma}+k \hat{\sigma}_{b}^{2}}$ possess the same reliability? (c) Are $\hat{w}=\frac{\hat{w}}{\hat{w}-\hat{w}^{\prime}}$ and $\hat{d}_{j}=\frac{c_{j} \Delta+r \lambda_{j} \hat{w}}{\Delta+r H \hat{w}+r^{2} \hat{w}^{2}}$ $(j=1,2)$, consistent estimators? One way of answering these questions is to find the variance of $\mathrm{T}_{i}$ after substituting 
in (1), $w, w^{\prime}, d_{1}, d_{2}$ by their estimates. A casual glance at this expression is enough to show that this is not mathematically feasible. It should also be noted that by taking $\mathbf{P}_{i}=\mathbf{w}_{\mathbf{i}}+\mathbf{w}^{\prime} \mathbf{Q}_{i}^{\prime}$, a new source of sampling variation is being introduced into the adjusted yield, and this is of special significance for small $\mathrm{v}$.

To show that at least, theoretically, the combined estimator obtained by the Rao method is better than the best linear combination of the intra-block and inter-block estimates, we will compare the theoretical variances of each. The theoretical variance of $T_{i}$ will be shown later to be:

$$
V\left(T_{i}\right)=\frac{k(v-1)-n_{1} d_{1}-n_{2} d_{2}}{\operatorname{vr}\left[w(k-1)+w^{\prime}\right]}
$$

Letting $\left[w(k-1)+w^{\prime}\right]=\eta$ and substituting (2.5) into (2.7), one obtains

$$
v\left(T_{i}\right)=\frac{k(v-1)}{v r \eta}-\frac{n_{1}}{v r \eta} \frac{c_{1} \Delta+r \lambda_{1} w}{\Delta+r H w+r^{2} w^{2}}-\frac{n_{2}}{v r \eta} \frac{c_{2} \Delta+r \lambda_{2} w}{\Delta+r H w+r^{2} w^{2}}
$$

By substituting (2.6) into (2.8) and simplifying

$$
\begin{aligned}
v\left(T_{i}\right)=\frac{k(v-1)}{v r \eta}- & \left\{\frac{n_{1}}{v r \eta} \frac{c_{1} \Delta\left(w-w^{\prime}\right)^{2}+r \lambda_{1} w^{\prime}\left(w^{-} w^{\prime}\right)}{\Delta\left(w-w^{\prime}\right)^{2}+r H w^{\prime}\left(w-w^{\prime}\right)+r^{2} w^{\prime 2}}\right. \\
& \left.+\frac{n_{2}}{v r \eta} \frac{c_{2} \Delta\left(w-w^{\prime}\right)^{2}+r \lambda_{2} w^{\prime}\left(w-w^{\prime}\right)}{\Delta\left(w-w^{\prime}\right)^{2}+r H w^{\prime}\left(w-w^{\prime}\right)+r^{2} w^{\prime 2}}\right\} .
\end{aligned}
$$


Now by collecting like terms, (2.9) may be written as

$$
V\left(T_{i}\right)=\frac{k(v-1)}{\operatorname{vr} \eta}-\frac{\left(n_{1} c_{1}+n_{2} c_{2}\right) \Delta\left(w-w^{\prime}\right)^{2}+r w^{\prime}\left(w-w^{\prime}\right)\left(n_{1} \lambda_{1}+n_{2} \lambda_{2}\right)}{\operatorname{vr} \eta\left[\Delta\left(w-w^{\prime}\right)^{2}+r H w^{\prime}\left(w-w^{\prime}\right)+r^{2} w^{\prime 2}\right]} .
$$

Then by letting $\left(\mathrm{n}_{1} \mathrm{c}_{1}+\mathrm{n}_{2} \mathrm{c}_{2}\right)=\varphi, \mathrm{u}=\frac{\mathrm{w}^{\prime}}{\mathrm{w}}$, and since $\left(n_{1} \lambda_{1}+n_{2} \lambda_{2}\right)=r(k-1)=a,(2.10)$ may be written as

$$
v\left(T_{i}\right)=\frac{k(v-1)}{v r \eta}-\frac{\varphi \Delta(1-u)^{2}+r a u(1-u)}{\operatorname{vr} \eta\left[\Delta(1-u)^{2}+r H u(1-u)+r^{2} u^{2}\right]} .
$$

Since

$$
u=\frac{w^{\prime}}{w}=\frac{\sigma^{2}}{\sigma^{2}+k \sigma_{b}^{2}}=\frac{1}{1+k \frac{\sigma_{b}^{2}}{\sigma^{2}}}=\frac{1}{1+k R},
$$

the following inequalities are obtained

$$
1<\mathrm{R}<\infty \quad \text { and } \quad 0<\mathrm{u}<\frac{1}{1+\mathrm{k}} \text {. }
$$

Now by substituting for $\eta$, after some manipulation (2.11) becomes

$$
\begin{aligned}
& \operatorname{vwV}\left(T_{i}\right)=\frac{k(v-1)}{r(k-1+u)}-\frac{(\varphi \Delta-r a) u^{2}-(2 \varphi \Delta-r a) u+\varphi \Delta}{r(k-1+u)\left[\left(\Delta+r^{2}-r H\right) u^{2}+(r H-2 \Delta) u+\Delta\right]}, \\
& =\frac{\left.k(v-1)\left[\Delta+r^{2}-r H\right) u^{2}+(r H-2 \Delta) u+\Delta\right]-(\varphi \Delta-r a) u^{2}+(2 \varphi \Delta-r a) u-\varphi \Delta}{r(k-I+u)\left[\left(\Delta+r^{2}-r H\right) u^{2}+(r H-2 \Delta) u+\Delta\right]} .
\end{aligned}
$$

Finally, letting $\mathrm{A}=\Delta+r^{2}-r H$, $\operatorname{vwv}\left(T_{i}\right)=\frac{[A k(v-1)-\phi \Delta+r a] u^{2}+[k(v-1)(r H-2 \Delta+2 \varphi \Delta-r a] u+k(v-1) \Delta-\phi \Delta}{r(k-1+u)\left[A u^{2}+(r H-2 A) u+\Delta\right]}$. 
Now if the intra-estimate $\hat{t}_{i}$ and the inter-estimate $\hat{t}_{i}$ of $t_{i}$ are combined, the best linear theoretical estimate is

$$
\hat{\tau}_{i}=\frac{\hat{t}_{i} v\left(\hat{t}_{i}^{\prime}\right)+\hat{t}_{i}^{\prime} v\left(\hat{t}_{i}\right)}{v\left(\hat{t}_{i}^{\prime}\right)+v\left(\hat{t}_{i}\right)}
$$

and

$$
v\left(\hat{\tau}_{i}\right)=\frac{v\left(\hat{t}_{i}\right) v\left(\hat{t}_{i}^{\prime}\right)}{v\left(\hat{t}_{i}^{\prime}\right)+v\left(\hat{t}_{i}\right)}
$$

It will be shown later that:

$$
\begin{aligned}
& v\left(\hat{t}_{i}\right)=\frac{k(v-1)-n_{1} c_{1}-n_{2} c_{2}}{a v w}, \\
& v\left(\hat{t}_{i}^{\prime}\right)=\frac{k(v-1)-n_{1} c_{1}-n_{2} c_{2}}{r v w^{\prime}},
\end{aligned}
$$

where

$$
c_{j}^{\prime}=\frac{\Delta c_{j}-r \lambda_{j}}{\Delta-r H+r^{2}}=\frac{\Delta c_{j}-r \lambda_{j}}{A}
$$

Substituting (2.16) and (2.17) into (2.15) one obtains

$$
\mathrm{v}\left(\hat{\tau}_{i}\right)=\frac{\left[\frac{\mathrm{k}(\mathrm{v}-1)-\mathrm{n}_{1} \mathrm{c}_{1}^{\prime}-\mathrm{n}_{2} \mathrm{c}_{2}^{\prime}}{r v w^{\prime}}\right]\left[\frac{k(\mathrm{v}-1)-\mathrm{n}_{1} \mathrm{c}_{1}-\mathrm{n}_{2} \mathrm{c}_{2}}{\mathrm{avw}}\right]}{\left[\frac{\mathrm{k}(\mathrm{v}-1)-\mathrm{n}_{1} \mathrm{c}_{1}^{\prime}-\mathrm{n}_{2} \mathrm{c}_{2}^{\prime}}{r v w^{\prime}}\right]+\left[\frac{k(v-1)-\mathrm{n}_{1} \mathrm{c}_{1}-\mathrm{n}_{2} \mathrm{c}_{2}}{\mathrm{avw}}\right]} \cdot
$$


Now from (2.18)

$$
\begin{aligned}
& n_{1} c_{1}^{\prime}+n_{2} c_{2}^{\prime}= n_{1} \frac{c_{1} \Delta-r \lambda_{1}}{A}+n_{2} \frac{c_{2} \Delta-r \lambda_{2}}{A}=\frac{\Delta}{A}\left(n_{1} c_{1}+n_{2} c_{2}\right) \\
& \quad-\frac{r}{A}\left(n_{1} \lambda_{1}+n_{2} \lambda_{2}\right) \\
&=\frac{\Delta \varphi-r a}{A} .
\end{aligned}
$$

Substituting (2.20) into (2.19),

$$
\begin{aligned}
& v\left(\hat{\tau}_{i}\right)=\frac{\left[k(v-1)-\frac{\Delta \varphi-r a}{A}\right][k(v-1)-\varphi]}{\operatorname{avw}\left[k(v-1)-\frac{\Delta \varphi r a}{A}\right]+r v w^{\prime}[k(v-1)-\varphi]}, \\
& =\frac{[A k(v-1)-\Delta \varphi+r a][k(v-1)-\varphi]}{\operatorname{avw}[A k(v-1)-\Delta \varphi+r a]+r v w^{\prime} A[k(v-1)-\varphi]} .
\end{aligned}
$$

Then

$$
\operatorname{vwV}\left(\hat{\tau}_{i}\right)=\frac{[A k(v-1)-\Delta \varphi+r a][k(v-1)-\varphi]}{r A[k(v-1)-\varphi] u+a[A k(v-1)-\Delta \varphi+r a]}
$$

A numerical study of the difference $y=\operatorname{vw}\left[\operatorname{var}\left(T_{i}\right)-\operatorname{Var}\left(\hat{\tau}_{i}\right)\right]$ has been conducted for the 68 Regular Group divisible experiments listed in reference (1). The range of $\mathrm{u}$ from 0 to $\frac{1}{1+k}$ is divided into ten equal intervals and eleven numerical values have been computed for the difference $y$, and for the percentage difference $s=y /\left[v \cdot v\left(\hat{\tau}_{i}\right)\right]$. The results show that $y$ is always negative, and the difference in absolute value increases as the ratio 
$\frac{\sigma_{b}^{2}}{\sigma^{2}}$ gets closer to one. The difference is zero when $\frac{\sigma_{b}^{2}}{\sigma^{2}} \rightarrow \infty$. Although the percentage difference is very small, this shows that the Rao method is theoretically better than obtaining, first, a separate intra- and inter-estimate, and then combining. The results for $S=\frac{1}{\sigma^{2}}$ [percentage gain due to Rao method] appear in Table 1.

In brief, one can say that, theoretically, the Rao method has some desirable properties. It constitutes the best way, knownso far, for getting a linear combined estimate; it is, relatively, simple to apply; and it is very general. But, the formula it produces contains a rather complicated function of unknown parameters which have to be estimated. Thus, the desirable theoretical properties of Rao's method are not likely to stand up in practice. Many valid questions arise about its reliability in practice, and it appears that the only amnesty it has is that it is very difficult to show mathematically if it is good or not. 


\section{THEOREM 1}

In this section a theorem will be proved concerning a new method of combining two independent unbiased estimates. It will also be shown how much improvement one can hope to get by combining two independent estimates. Finally, it will be shown how much of the improvement this new method utilizes.

Consider the $\mathrm{n}$ independent parameters $\tau_{1}, \tau_{2}, \ldots, \tau_{\mathrm{n}}$ and suppose that for each $\tau_{i}$ there exist two independent unbiased estimates $U_{i}$ and $x_{i}$, where $U_{i} \cap N\left(\tau_{i}, \rho \sigma^{2}\right)$ and $x_{i} \cap N\left(\tau_{i}, p ! \sigma^{\prime 2}\right)$. Suppose also that independently of the $\mathrm{x}_{i}$ 's and $\mathrm{U}_{i}$ 's, there exist two unbiased estimates $\mathrm{s}^{2}$ and $\mathrm{s}^{\prime 2}$ for $\sigma^{2}, \sigma^{\prime 2}$ respectively, where $s^{2} \cap \chi^{2} \frac{\sigma^{2}}{f}$ and $s^{\prime 2} \cap \chi^{2} \frac{\sigma^{\prime 2}}{f^{\prime}}$, then:

(1) the combined estimate:

$$
\hat{\tau}_{i}=U_{i}+\frac{\theta(m-2) s^{2}}{\sum_{j=1}^{m}\left(x_{j}-U_{j}\right)^{2}}\left(x_{i}-U_{i}\right), \quad m \leq n,
$$

is unbiased and uniformly better than $\mathrm{U}_{i}$, and 
(2) the combined estimate:

$$
\hat{\tau}_{i}=x_{i}+\frac{\theta^{\prime}(m-2) s^{\prime 2}}{\sum_{j=1}^{m}\left(U_{j}-x_{j}\right)^{2}}\left(u_{i}-x_{i}\right), \quad m \leq n,
$$

is unbiased and uniformly better than $\mathbf{x}_{i} \cdot \theta$ and $\theta^{\prime}$ are constants.

Proof: Since $\mathrm{U}_{1}, \mathrm{U}_{2}, \ldots, \mathrm{U}_{\mathrm{n}} ; \mathrm{x}_{1}, \mathrm{x}_{2}, \ldots, \mathrm{x}_{\mathrm{n}} ; \mathrm{s}^{2}, \mathrm{~s}^{12}$ are independent, and $\mathbf{E}\left(\mathrm{U}_{i}\right)=\mathrm{E}\left(\mathrm{x}_{i}\right)=\tau_{i}, i=1,2, \ldots, \mathrm{n}$,

$$
E\left(\hat{\tau}_{i}\right)=\tau_{i}+\theta(m-2) E\left(s^{2}\right) \cdot E \frac{x_{i}-U_{i}}{\sum_{j=1}^{m}\left(x_{j}-U_{j}\right)^{2}}
$$

Let $\mathrm{x}_{i}-\mathrm{U}_{i}=\mathrm{z}_{i}$, then $z_{i} \frown \mathrm{N}\left(0, \mathrm{~V}\left(\mathrm{U}_{i}\right)+\mathrm{V}\left(\mathrm{x}_{i}\right)\right)$. The joint distribution of $z_{1}, z_{2}, \ldots, z_{m}$ is of the form:

$$
f\left(z_{1}\right), f\left(z_{2}\right), \ldots, f\left(z_{m}\right)=A e^{-B \sum_{j=1}^{m} z_{j}^{2}},-\infty<z_{i}<+\infty,
$$

an even function of $z_{1}, z_{2}, \ldots, z_{m}$; the expectation, therefore, of any odd function of $z_{1}, z_{2}, \ldots, z_{m}$ is zero. If $z_{i}$ belongs to the subset $z_{1}, z_{2}, \ldots, z_{m}$, then

$$
E \frac{z_{i}}{\sum_{j=1}^{m} z_{j}^{2}}=0
$$


Hence $\mathbf{E}\left(\hat{\tau}_{i}\right)=\tau_{i}$ and $\hat{\tau}_{i}$ is the unbiased estimate for $\tau_{i}$. Now

$$
\begin{aligned}
& V\left(\hat{\tau}_{i}\right)=E\left(\hat{\tau}_{i}-\tau_{i}\right)^{2}=E\left[\left(u_{i}-\tau_{i}\right)+\frac{\theta(m-2) s^{2}}{\sum_{j=1}^{m}\left(x_{j}-U_{j}\right)^{2}}\left(x_{i}-u_{i}\right]^{2}\right. \\
& =E\left(U_{i}-\tau_{i}\right)^{2}+\theta^{2}(m-2)^{2} \cdot E\left[\frac{s^{2}\left(x_{i}-U_{i}\right)}{\sum_{j=1}^{m}\left(x_{j}-U_{j}\right)^{2}}\right]^{2}+2 \theta(m-2) \cdot E\left[\frac{s^{2}\left(x_{i}-U_{i}\right)\left(U_{i}-\tau_{i}\right)}{\sum_{j=1}^{m}\left(U_{j}-x_{j}\right)^{2}}\right] \\
& =V\left(U_{i}\right)+\theta^{2}(m-2)^{2} \cdot E\left(s^{4}\right) \cdot E \frac{z_{i}^{2}}{\left(\sum_{j=1}^{m} z_{j}^{2}\right)^{2}}+2 \theta(m-2) \cdot E\left(s^{2}\right) \cdot E \frac{z_{i}\left(U_{i}-\tau_{i}\right)}{\sum_{j=1}^{m} z_{j}^{2}},
\end{aligned}
$$

where

$$
E\left(s^{4}\right)=\left(\frac{\sigma^{2}}{f}\right)^{2} E\left(X_{f}^{2}\right)^{2}=\frac{\sigma^{4} f(f+2)}{f^{2}}=\frac{\sigma^{4}(f+2)}{f},
$$

and

$$
E \frac{z_{i}^{2}}{\left(\sum_{j=1}^{m} z_{j}^{2}\right)^{2}}=\frac{1}{m} E\left[\frac{z_{1}^{2}+z_{2}^{2}+\ldots+z_{m}^{2}}{\left(\sum_{j=1}^{m} z_{j}^{2}\right)^{2}}\right],
$$

because of the symmetry of the $z_{i}$ 's; that is,

$$
E \frac{z_{i}^{2}}{\left(\sum_{j=1}^{m} z_{j}^{2}\right)^{2}}=E \frac{z_{k}^{2}}{\left(\sum_{j=1}^{m} z_{j}^{2}\right)^{2}}, k=1,2, \ldots, m \text {. }
$$


Hence:

$$
\begin{aligned}
& E \frac{z_{i}^{2}}{\left(\sum_{j=1}^{m} z_{j}^{2}\right)^{2}}=\frac{1}{m} \cdot E \frac{\sum_{j=1}^{m} z_{j}^{2}}{\left(\sum_{j=1}^{m} z_{j}^{2}\right)^{2}}=\frac{1}{m} \cdot E \frac{1}{\sum_{j=1}^{m} z_{j}^{2}}, \\
& =\frac{1}{m} \cdot E \frac{1}{\left(V\left(U_{i}\right)+V\left(x_{i}\right)\right) X_{m}^{2}}=\frac{1}{m(m-2)\left(V\left(U_{i}\right)+V\left(x_{i}\right)\right)}
\end{aligned}
$$

Then

$$
\begin{aligned}
& E \frac{z_{i}\left(U_{i}-\tau_{i}\right)}{\sum_{j=1}^{m} z_{j}^{2}}=E_{z_{1}}, z_{2}, \ldots, z_{m}\left[E_{U_{i}} \mid z_{1}, z_{2}, \ldots, z_{m} \frac{z_{i}\left(U_{i}-\tau_{i}\right)}{\sum_{j=1}^{m} z_{j}^{2}}\right], \\
& =E_{z_{1}}, z_{2}, \ldots, z_{m}\left[\frac{z_{i}}{\sum_{j=1}^{m} z_{j}^{2}} \cdot E_{U_{i}} \mid z_{1}, z_{2}, \ldots, z_{m}\left(U_{i}-\tau_{i}\right)\right] .
\end{aligned}
$$

Consider now the multivariate normal vector $\left[u_{i}, z_{1}, z_{2}, \ldots, z_{i}, \ldots, z_{m}\right]$; its mean vector is $\left[\tau_{i}, 0,0, \ldots, 0\right]$ and the variance covariance matrix is: 


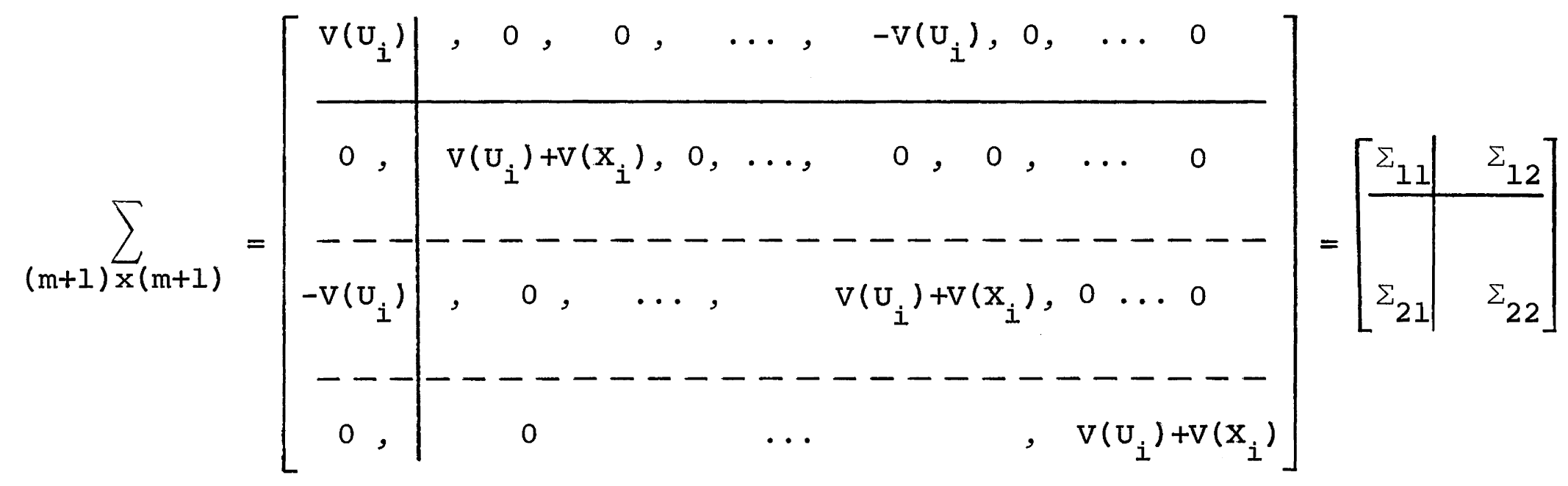


Since the conditional mean of $U_{i} \mid z_{1}, z_{2}, \ldots, z_{m}$ is:

$$
\begin{aligned}
\tau_{i}+ & \Sigma_{12} \Sigma_{22}^{-1} \underline{z}=\tau_{i}+\left(0,0, \ldots,-V\left(U_{i}\right), 0, \ldots, 0\right)\left[\left(V\left(U_{i}\right)+V\left(x_{i}\right)\right) I_{m}\right]^{-1} \cdot \underline{z} \\
& =\tau_{i}+\left(0,0, \ldots, \frac{-V\left(U_{i}\right)}{V\left(U_{i}\right)+V\left(x_{i}\right)}, 0, \ldots, 0\right) \underline{z}=\tau_{i}-\frac{V\left(U_{i}\right) \cdot z_{i}}{V\left(U_{i}\right)+V\left(x_{i}\right)},
\end{aligned}
$$

then

$$
E_{U_{i}} \mid z_{1}, z_{2}, \ldots, z_{m}\left(U_{i}-\tau_{i}\right)=-\frac{V\left(U_{i}\right) \cdot z_{i}}{V\left(U_{i}\right)+V\left(x_{i}\right)} .
$$

Substituting (3.10) into (3.9) one obtains:

$$
\begin{aligned}
& E \frac{z_{i}\left(U_{i}-\tau_{i}\right)}{\sum_{j=1}^{m} z_{j}^{2}}=E_{z_{1} \ldots z_{m}}\left[\frac{-V\left(U_{i}\right)}{V\left(U_{i}\right)+V\left(x_{i}\right)} \frac{z_{i}^{2}}{\sum_{j=1}^{m} z_{j}^{2}}\right], \\
& =\frac{-V\left(U_{i}\right)}{V\left(U_{i}\right)+V\left(x_{i}\right)} E_{z_{1}} \ldots z_{m} \frac{z_{i}^{2}}{\sum_{j=1}^{m} z_{j}^{2}}=\frac{-V\left(U_{i}\right)}{m\left(V\left(U_{i}\right)+V\left(x_{i}\right)\right)} E\left[\frac{z_{1}^{2}+z_{2}^{2}+\ldots+z_{m}^{2}}{\sum_{j=1}^{m} z_{j}^{2}}\right], \\
& =\frac{-V\left(U_{i}\right)}{m\left(V\left(U_{i}\right)+V\left(x_{i}\right)\right)},
\end{aligned}
$$

making use of the symmetry of $z_{1}, z_{2}, \ldots, z_{n}$. Substituting $(3.6),(3.8)$, and (3.11) into (3.5), one obtains 


$$
\begin{aligned}
V\left(\hat{\tau}_{i}\right) & =V\left(U_{i}\right)+\frac{\theta^{2} \sigma^{4}(m-2)^{2}(f+2)}{f m(m-2)\left(V\left(U_{i}\right)+V\left(X_{i}\right)\right)}-2 \frac{\theta \sigma^{2} V\left(U_{i}\right)(m-2)}{m\left[V\left(U_{i}\right)+V\left(X_{i}\right)\right]}, \\
& =V\left(U_{i}\right)+\frac{\theta^{2}(m-2)(f+2)\left[V\left(U_{i}\right)\right]^{2}}{\rho^{2} f m\left[V\left(U_{i}\right)+V\left(X_{i}\right)\right]}-2 \frac{\theta(m-2)\left[V\left(U_{i}\right)\right]^{2}}{\rho m\left[V\left(U_{i}\right)+V\left(X_{i}\right)\right]}, \\
& =V\left(U_{i}\right)+\frac{\theta(m-2)\left[V\left(U_{i}\right)\right]^{2}}{m \rho\left[V\left(U_{i}\right)+V\left(X_{i}\right)\right]}\left[\frac{\theta(f+2)}{\rho f}-2\right] .
\end{aligned}
$$

The value of $\theta$ which makes the second term above the most negative is $\theta=\frac{\rho f}{f+2}$, or $\frac{\theta}{\rho}=\frac{f}{f+2}$, substituting for $\frac{\theta}{\rho}$ one obtaine: $V\left(\hat{\tau}_{i}\right)=V\left(U_{i}\right)-\left(1-\frac{2}{m}\right)\left(1-\frac{2}{f+2}\right) \frac{\left[V\left(U_{i}\right)\right]^{2}}{V\left(U_{i}\right)+V\left(X_{i}\right)} \leq V\left(U_{i}\right), \quad$ provided $m \geq 2$.

The proof of the second part follows similarly.

\subsection{Remarks}

(a) The best linear unbiased combined estimate $\hat{\tau}_{i}$ of $\tau_{i}$ is:

$$
\hat{\tau}_{i}=\frac{U_{i} V\left(X_{i}\right)+x_{i} V\left(U_{i}\right)}{V\left(U_{i}\right)+V\left(x_{i}\right)}
$$

with the minimum variance:

$$
V\left(\hat{\tau}_{i}\right)=\frac{V\left(U_{i}\right) \cdot V\left(x_{i}\right)}{V\left(U_{i}\right)+V\left(x_{i}\right)}=V\left(U_{i}\right)-\frac{\left[V\left(U_{i}\right)\right]^{2}}{V\left(U_{i}\right)+V\left(x_{i}\right)}
$$


This shows that the most improvement one can hope to get by combining the two independent estimates of $\tau_{i}$, namely $U_{i}$ and $x_{i}$ is the quantity $\frac{\left[V\left(U_{i}\right)\right]^{2}}{V\left(U_{i}\right)+V\left(X_{i}\right)}$. It should be noticed that the above suggested estimate recovers $\frac{(m-2) f}{m(f+2)}$ of the most improvement possible $\frac{\left[V\left(U_{i}\right)\right]^{2}}{V\left(U_{i}\right)+V\left(X_{i}\right)} \cdot$ The fraction $\frac{(m-2) f}{m(f+2)}=\left(1-\frac{2}{m}\right)\left(1-\frac{2}{f+2}\right) \rightarrow 1$ when $m$ and $f$ are large. It is obvious now that the larger $m$ is, the better the new estimate will be. This means that we should take $m=n$ whenever it is possible.

(b) The variance of the combined estimate $\tau_{i}^{\prime}$ is:

$$
V(\tau \dot{i})=V\left(X_{i}\right)-\frac{(m-2) f^{\prime}\left[V\left(X_{i}\right)\right]^{2}}{\left(f^{\prime}+2\right) m\left[V\left(U_{i}\right)+V\left(X_{i}\right)\right]} \text {. }
$$

Subtracting (3.1.2) from (3.12),

$V\left(\tau_{i}\right)-V\left(\tau_{i}^{\prime}\right)=\left[V\left(U_{i}\right)-V\left(X_{i}\right)\right]$

$$
+\frac{m-2}{m\left[V\left(U_{i}\right)+V\left(X_{i}\right)\right]} \frac{f^{\prime}\left[V\left(X_{i}\right)\right]^{2}}{f^{\prime}+2}-\frac{f\left[V\left(U_{i}\right)\right]^{2}}{f+2}, .
$$

Simplifying the R.H.S., the sign of $V\left(\hat{\tau}_{i}\right)-V\left(\hat{\tau}_{i}^{\prime}\right)$ is the same as the sign of: 


$$
\begin{aligned}
& m\left(f^{\prime}+2\right)(f+2)\left[V^{2}\left(U_{i}\right)-V^{2}\left(X_{i}\right)\right]+(m-2)\left[f^{\prime}(f+2) v^{2}\left(X_{i}\right)-f\left(f^{\prime}+2\right) v^{2}\left(U_{i}\right)\right] \\
& \quad=2 v^{2}\left(U_{i}\right)\left[m f^{\prime}+f^{\prime}+2 f+2 m\right]-2 v^{2}\left(X_{i}\right)\left[m f+f f^{\prime}+2 f^{\prime}+2 m\right] \\
& \quad=2(m+f)\left(f^{\prime}+2\right) v^{2}\left(U_{i}\right)-2\left(m^{\prime}+f^{\prime}\right)(f+2) v^{2}\left(X_{i}\right) .
\end{aligned}
$$

Thus:

$$
\begin{aligned}
& V\left(\hat{\tau}_{i}\right)>V\left(\hat{\tau}_{i}^{\prime}\right), \quad \text { if } \frac{V^{2}\left(U_{i}\right)}{V^{2}\left(X_{i}\right)}>\frac{\left(f^{\prime}+m\right)(f+2)}{(f+m)\left(f^{\prime}+2\right)} \quad \text { and } \\
& V\left(\hat{\tau}_{i}\right)<V\left(\hat{\tau}_{i}^{\prime}\right), \quad \text { if } \frac{V^{2}\left(U_{i}\right)}{V^{2}\left(X_{i}\right)}<\frac{\left(f^{\prime}+m\right)(f+2)}{(f+m)\left(f^{\prime}+2\right)} .
\end{aligned}
$$

\subsection{Use of Theorem 1}

This theorem could be utilized for obtaining better estimates for a number $\mathrm{n}$ of independent comparisons between the treatments in any incomplete block design, provided that each comparison has the same variance, and the number of such comparisons is more than 2.

It could also be used to combine the estimates of the same treatment from two independent similar experiments, provided that the interaction over time and location is negligible.

Also, if one is in doubt about the homogeneity assumption when a randomized block design has been utilized, the blocks could be divided into two homogeneous groups, and 
then obtain estimates for the $\mathrm{v}-1$ comparisons among treatments within each group. Then combine these estimates by theorem 1. The gain in the accuracy of this estimate increases as the heterogeneity between the two groups increases. Again, the block by treatment interaction must be negligible. 
$-24-$

4. IEMMA 1

If two independent random variables $\mathrm{X}$ and $\mathrm{Y}$ have density functions:

$$
\begin{aligned}
& f(x)=\frac{1}{\Gamma\left(\alpha_{1}+1\right) \beta_{1} \alpha_{1}+1} x^{\alpha_{1}} e^{-x / \beta_{1}} d x \quad x>0, \beta_{1}>0, \\
& f(y)=\frac{1}{\Gamma\left(\alpha_{2}+1\right) \beta_{2} \alpha_{2}+1} y^{\alpha_{2}} e^{-y / \beta_{2}} d y \quad y>0, \beta_{2}>0 .
\end{aligned}
$$

Then:

$$
\mathbf{E}\left(\frac{1}{X+Y}\right)^{r}=\frac{\Gamma\left(\alpha_{1}+\alpha_{2}+2-r\right)}{\Gamma\left(\alpha_{1}+1\right) \Gamma\left(\alpha_{2}+1\right)} \int_{0}^{1} \frac{u^{\alpha_{1}}(1-u)^{\alpha_{2}}}{\left[\beta_{1} u+\beta_{2}(1-u)\right]^{r}} d u
$$

Proof:

$$
\mathbf{E}\left(\frac{1}{X+Y}\right)^{r}=c t \cdot \int_{0}^{\infty} \int_{0}^{\infty} \frac{1}{(x+y)^{r}} x^{\alpha} e^{-x / \beta_{1}} y^{\alpha} 2 e^{-y / \beta_{2}} d x d y \text {, }
$$

where

$$
\text { ct. }=\frac{1}{\Gamma\left(\alpha_{1}+1\right) \Gamma\left(\alpha_{2}+1\right) \beta_{1}^{\alpha_{1}+1} \beta_{2}^{\alpha_{2}+1}} \text {. }
$$

Let $\frac{x}{\beta_{1}}=z_{1}, \frac{y}{\beta_{2}}=z_{2}, d x=\beta_{1} d z_{1}, d y=\beta_{2} d z_{2}$, 
then

$$
\begin{array}{r}
\mathbf{E}\left(\frac{1}{x+y}\right)^{r}=C t \cdot \int_{0}^{\infty} \int_{0}^{\infty} \frac{1}{\left(\beta_{1} z_{1}+\beta_{2} z_{2}\right)^{r}} z_{1}^{\alpha_{1}} e^{-z_{1}} z_{2}^{\alpha} e^{-z_{2}} \beta_{1}^{\alpha+1} \beta_{2}^{\alpha+1} \\
\cdot d z_{1} d z_{2} \cdot(4.4)
\end{array}
$$

Now let $u=\frac{z_{1}}{z_{1}+z_{2}}, \quad z_{2}=z_{2}$, then:

$\mathbf{E}\left(\frac{1}{x+y}\right)^{r}=c t \cdot \beta_{1}^{\alpha+1} \beta_{2}^{\alpha} \int_{0}^{\infty} \int_{0}^{1} \frac{1}{\left.\frac{\beta_{1} u z_{2}}{1-u}+\beta_{2} z_{2}\right)^{r}}\left(\frac{u z_{2}}{1-u}\right)^{\alpha} e^{-\frac{u z_{2}}{1-u}}$
$\cdot z_{2}^{\alpha^{\alpha}} e^{-z_{2}} \frac{z_{2}}{(1-u)^{2}} d u d z_{2}$,

$=c t \cdot \beta_{1}^{\alpha_{1}+1} \beta_{2}^{\alpha_{2}+1} \int_{0}^{\infty} \int_{0}^{1} \frac{(1-u)^{r}}{\left[\beta_{1} u z_{2}+\beta_{2} z_{2}(1-u)\right]^{r}}\left(\frac{u}{1-u}\right)^{\alpha}{ }^{\alpha} z_{2}^{\alpha_{1}+\alpha_{2}+1}$

$$
e^{-z_{2}} e^{-\frac{u z}{1-u}} \frac{1}{(1-u)^{2}} d u d z_{2}
$$

Let $z_{2}=(1-u) v, u=u$, then: 
$-26-$

$$
\begin{aligned}
& \mathbf{E}\left(\frac{1}{x+y}\right)^{r}=c t \cdot \beta_{1}^{\alpha} 1^{+1} \beta_{2}^{\alpha+1} \int_{0}^{\infty} \int_{0}^{1} \frac{(1-u)^{r}}{\left[\beta_{1} u(1-u) v+\beta_{2} v(1-u)^{2}\right]^{r}} \\
& \text { - }\left(\frac{u}{1-u}\right)^{\alpha_{1}}(1-u)^{\alpha_{1}+\alpha_{2}+1} v^{\alpha_{1}+\alpha_{2}+1} e^{-v(1-u)} e^{-u v} \frac{d u}{(1-u)^{2}}(1-u) d v \text {, } \\
& =\text { ct. } \beta_{1}^{\alpha} 1^{+1} \beta_{2}^{\alpha} 2^{+1} \int_{0}^{\infty} \int_{0}^{1} \frac{(1-u)^{r}}{v^{r}(1-u)^{r}\left[\beta_{1} u+\beta_{2}(1-u)\right]^{r}} u^{\alpha_{1}}(1-u)^{\alpha} \\
& v^{\alpha_{1}+\alpha}{ }^{+1} e^{-v} d u d v \\
& =\text { ct. } \beta_{1}^{\alpha_{1}+1} \beta_{2}^{\alpha_{2}+1} \int_{0}^{\infty} v^{\alpha_{1}+\alpha_{2}-r+1} e^{-v} d v \int_{0}^{1} \frac{1}{\left[\beta_{1} u+\beta_{2}(1-u)\right]^{r}} \\
& u^{\alpha}{ }^{\alpha}(1-u)^{\alpha} d u \text {, } \\
& \text { ct. } \beta_{1}^{\alpha_{1}+1} \beta_{2}^{\alpha_{2}+1} \Gamma\left(\alpha_{1}+\alpha_{2}+2-r\right) \int_{0}^{1} \frac{1}{\left[\beta_{1} u+\beta_{2}(1-u)\right]^{r}} u^{\alpha_{1}}(1-u)^{\alpha_{2}} d u \text {, } \\
& =\frac{\Gamma\left(\alpha_{1}+\alpha_{2}+2-r\right)}{\Gamma\left(\alpha_{1}+1\right) \Gamma\left(\alpha_{2}+1\right)} \int_{0}^{1} \frac{u^{\alpha_{1}}(1-u)^{\alpha_{2}}}{\left[\beta_{1} u+\beta_{2}(1-u)\right]^{r}} d u \text {. }
\end{aligned}
$$

4.1 Corollary

$$
\begin{aligned}
& \text { Since } \beta_{2}>0,0 \leq \mathrm{u} \leq 1 \text {, and assuming } \beta_{1}>\beta_{2} \text {, then: } \\
& \beta_{1} \mathrm{u}<\beta_{1} \mathrm{u}-\beta_{2} \mathrm{u}+\beta_{2}<\beta_{1}
\end{aligned}
$$

or

(4.1.1)

$$
\beta_{2}<\beta_{1} u-\beta_{2} u+\beta_{2}<\beta_{1} .
$$


Then

$\frac{\Gamma\left(\alpha_{1}+\alpha_{2}+2-r\right) \beta\left(\alpha_{1}+1, \alpha_{2}+1\right)}{\Gamma\left(\alpha_{1}+1\right) \Gamma\left(\alpha_{2}+1\right) \beta_{1}^{r}}<E\left(\frac{1}{X+Y}\right)^{r}<\frac{\Gamma\left(\alpha_{1}+\alpha_{2}+2-r\right) \beta\left(\alpha_{1}+1-r, \alpha_{2}+1\right)}{\Gamma\left(\alpha_{1}+1\right) \Gamma\left(\alpha_{2}+1\right) \beta_{1}^{r}}$

and

$\frac{\Gamma\left(\alpha_{1}+\alpha_{2}+2-r\right) \beta\left(\alpha_{1}+1, \alpha_{2}+1\right)}{\Gamma\left(\alpha_{1}+1\right) \Gamma\left(\alpha_{2}+1\right) \beta_{1}^{r}}<E\left(\frac{1}{X+Y}\right)^{r}<\frac{\Gamma\left(\alpha_{1}+\alpha_{2}+2-r\right) \beta\left(\alpha_{1}+1, \alpha_{2}+1\right)}{\Gamma\left(\alpha_{1}+1\right) \Gamma\left(\alpha_{2}+1\right) \beta_{2}^{r}}$,

which can be written respectively as:

$$
\frac{\Gamma\left(\alpha_{1}+\alpha_{2}+2-r\right)}{\Gamma\left(\alpha_{1}+\alpha_{2}+2\right) \beta_{1}^{r}}<E\left(\frac{1}{X+Y}\right)^{r}<\frac{\Gamma\left(\alpha_{1}+1-r\right)}{\beta_{1}^{r} \Gamma\left(\alpha_{1}+1\right)},
$$

and

$$
\frac{\Gamma\left(\alpha_{1}+\alpha_{2}+2-r\right)}{\Gamma\left(\alpha_{1}+\alpha_{2}+2\right) \beta_{1}^{r}}<E\left(\frac{1}{X+Y}\right)^{r}<\frac{\Gamma\left(\alpha_{1}+\alpha_{2}+2-r\right)}{\Gamma\left(\alpha_{1}+\alpha_{2}+2\right) \beta_{2}^{r}} .
$$




\section{IEMMA 2}

Let $z_{1}, z_{2}, \ldots, z_{n}$ have the joint multivariate normal distribution, with mean vector $\underline{\mu}^{\prime}=\underline{0}$ and variance covariance matrix $\Sigma$ with the diagonal element (a) and the element (b) otherwise. Then $\sum_{i=1}^{n} z_{i}^{2}$ can be considered as equivalent to the sum of two independent, Gamma distributed, random variables.

Proof: The characteristic function of $\sum_{i=1}^{n} z_{i}^{2}$ is: $\Phi_{\theta}\left(\sum z_{i}^{2}\right)=\int_{-\infty}^{\infty} \cdots \int_{-\infty}^{+\infty} \frac{|\Sigma|^{-\frac{1}{2}}}{\theta \underline{z}^{\prime} \underline{z}} e^{-\frac{1}{2} \underline{z}^{\prime} \Sigma^{-1} \underline{z}} d z_{1} \ldots d z_{n}$, $=\frac{|\Sigma|^{-\frac{1}{2}}}{(2 \pi)^{\frac{n}{2}}} \int_{-\infty}^{+\infty} \ldots \int_{-\infty}^{+\infty} e^{-\frac{1}{2}\left[\underline{z}^{\prime} \Sigma^{-1} \underline{z}-2 \theta \underline{z^{\prime}} \underline{z}\right]} d \underline{z}$,
$=\frac{\left.|\Sigma|\right|^{-\frac{1}{2}}}{(2 \pi)^{\frac{n}{2}}} \int_{-\infty}^{+\infty} \cdots \int_{-\infty}^{+\infty} e^{-\frac{1}{2} \underline{z}^{\prime}\left[\Sigma^{-1}-2 \theta I\right] \underline{z}} d \underline{z}$,

$$
=\frac{(2 \pi)^{\frac{n}{2}}}{\left|\Sigma^{-1}-2 \theta I\right|^{\frac{1}{2}}} \cdot \frac{|\Sigma|^{-\frac{1}{2}}}{(2 \pi)^{\frac{n}{2}}}=\frac{\left|\Sigma^{-1}\right|^{\frac{1}{2}}}{\left|\Sigma^{-1}-2 \theta I\right|^{\frac{1}{2}}}=\frac{1}{|I-2 \theta \Sigma|^{\frac{1}{2}}} \text {. }
$$


Since the inverse of an ( $n \times n)$ two element matrix, a along the diagonal and $b$ otherwise is an $(n \times n)$ two element matrix, d along the diagonal and $f$ off diagonal where:

$$
\mathrm{d}=\frac{-[(n-2) b+a]}{b^{2}(n-1)-a[(n-2) b+a]},
$$

and

$$
f=\frac{b}{b^{2}(n-1)-a[(n-2) b+a]} .
$$

It is known also that the determinant of such a matrix is:

$$
\Delta=(a-b)^{n-1}(a-b+n b)
$$

Substituting (5.4) into (5.1),

$$
|I-2 \theta \Sigma|^{\frac{1}{2}}=[1-2(a-b) \theta]^{\frac{n-1}{2}}[1-2(a+n b-b) \theta]^{\frac{1}{2}},
$$

and then

$$
\Phi_{\theta}\left(\sum_{i=1}^{n} z_{i}^{2}\right)=\frac{1}{[1-2(a-b) \theta]^{\frac{n-1}{2}}} \frac{1}{[1-2(a+n b-b) \theta]^{\frac{1}{2}}} .
$$

This means that $\sum_{i=1}^{n} z_{i}^{2}$ can be considered as the sum of two independent random variables $\mathrm{X}$ and $\mathrm{Y}$, where:

$$
\begin{array}{r}
f(x)=\frac{1}{[2(a-b)]^{\frac{n-1}{2}} \Gamma\left(\frac{n-1}{2}\right)} x^{\frac{n-3}{2}} e^{-x / 2(a-b)} d x, \\
x>0, \quad a-b>0,
\end{array}
$$


and

$$
\begin{gathered}
f(Y)=\frac{1}{2^{\frac{1}{2}}(a+n b-b)^{\frac{1}{2}} \Gamma\left(\frac{1}{2}\right)} y^{-\frac{1}{2}} e^{-y / 2(a+n b-b)} d y, \\
y>0, \quad a+n b-b>0
\end{gathered}
$$

5.1 Corollary 1

Using Lemma 2, one can write:

$$
\mathrm{E}\left(\frac{1}{\sum z_{i}^{2}}\right)=\mathrm{E}\left(\frac{1}{\mathrm{X}+\mathrm{Y}}\right)=\mathrm{E}\left[\frac{1}{\mathrm{X}}\left(1+\frac{\mathrm{Y}}{\mathrm{x}}\right)^{-1}\right]<\mathrm{E} \frac{1}{\mathrm{x}}=\frac{1}{(\mathrm{n}-3)(\mathrm{a}-\mathrm{b})},
$$

i.e.,

$$
\mathbf{E}\left(\frac{1}{\sum z_{i}^{2}}\right)<\frac{1}{(n-3)(a-b)}
$$

\subsection{Corollary 2}

Applying the results of Lemma 1 ,

$$
\begin{array}{ll}
\alpha_{1}=\frac{n-3}{2}, & \beta_{1}=2(a-b), \\
\alpha_{2}=\frac{-1}{2}, & \beta_{2}=2(a+n b-b) .
\end{array}
$$

Substituting (5.2.1) into (4.6),

$$
E\left(\frac{1}{\sum z_{i}^{2}}\right)^{r}=E\left(\frac{1}{X+Y}\right)^{r}=\frac{\Gamma\left(\frac{n}{2}-r\right)}{\Gamma\left(\frac{n-1}{2}\right) \Gamma\left(\frac{1}{2}\right)} \int_{0}^{l} \frac{u^{\frac{n-3}{2}}(1-u)^{-\frac{1}{2}}}{[-2 n b u+2(a+n b-b)]^{r}} d u .
$$


6. SOME RELATIONS IN B.I.B. DESIGNS

Let $\hat{t}_{i}$ denote the intra-block estimate of the $i-t h$ treatment effect and let $\hat{t}_{i}^{\prime}$ denote the corresponding interblock estimate, then

$$
\begin{gathered}
v\left(\hat{t}_{i}\right)=v_{1}=\frac{k(v-1)}{\lambda v^{2}} \sigma^{2}, \\
v\left(\hat{t}_{i}^{\prime}\right)=v_{2}=\frac{k(v-1)}{v(r-\lambda)} \sigma^{\prime 2}, \\
\operatorname{cov}\left(\hat{t}_{i}, \hat{t}_{j}\right)=c_{1}=\frac{-k}{\lambda v^{2}} \sigma^{2} \quad \text { for all } i \neq j,
\end{gathered}
$$

and

$$
\operatorname{cov}\left(\hat{t}_{i}^{\prime}, \hat{t}_{j}^{\prime}\right)=c_{2}=\frac{-k}{v(r-\lambda)} \sigma^{\prime 2} \text { for all } i \neq j
$$

The following relations hold:

$$
\begin{aligned}
& c_{1} v_{2}-v_{1} c_{2}=0, \\
& v_{1}+(v-1) c_{1}=0,
\end{aligned}
$$

and

$$
v_{2}+(v-1) c_{2}=0
$$

Then

$$
\mathrm{v}-\mathrm{c}=-\mathrm{vC},
$$

where $\mathrm{V}=\mathrm{v}_{1}+\mathrm{v}_{2}, \quad \mathrm{C}=\mathrm{c}_{1}+\mathrm{c}_{2}$, 
and

$$
\frac{v_{1}-C_{1}}{v_{1}}=\frac{v}{v-1}
$$

The intra-estimate of the i-th treatment is given in (6) as:

$$
\hat{t}_{i}=\frac{Q_{i}}{r E} \text {, }
$$

where $\mathbf{Q}_{i}$ is the adjusted treatment total, and the efficiency,

$$
E=\frac{(k-1) v}{k(v-1)}
$$

The inter-estimate of the i-th treatment is given in (11) as:

$$
\hat{t}_{i}^{\prime}=\frac{Q_{i}^{\prime}}{r(1-E)},
$$

where

$$
Q_{i}^{\prime}=Y_{i}-Q_{i}-r y, \ldots,
$$

$Y_{i}$. is the total yield of the $i-t h$ treatment, and $y$ is the overall mean. 


\section{THEOREM 2}

In B.I.B. designs, the combined estimate:

$$
\hat{\tau}_{i}=\hat{t}_{i}+\frac{k(v-4) s^{2}}{\lambda v(f+2) \sum_{i=1}^{v-1}\left(\hat{t}_{i}^{\prime}-\hat{t}_{i}\right)^{2}}\left(\hat{t}_{i}^{\prime}-\hat{t}_{i}\right),
$$

is unbiased for the $i$-th treatment effect $t_{i}$, and uniformly better than intra-block estimate provided $v>4$, where $\mathrm{s}^{2}$ and $f$ are the error sum of squares and the error degrees of freedom, respectively.

Proof: Consider first the estimate:

$$
\hat{\tau}_{i}=\hat{t}_{i}+\frac{\theta B s^{2}}{\sum_{j=1}^{n}\left(\hat{t}_{j}^{\prime}-\hat{t}_{j}\right)^{2}}\left(\hat{t}_{i}^{\prime}-\hat{t}_{i}\right), n<v,
$$

where $\theta=\frac{k(v-1)}{\lambda v^{2}}$, the coefficient of $\sigma^{2}$ in $\operatorname{var}\left(\hat{t}_{i}\right), s^{2}$ is the error mean square, and $B$ is to be determined later. Now

$$
E\left(\hat{\tau}_{i}\right)=t_{i}+\theta B \sigma^{2} E\left(\frac{z_{i}}{\sum_{j=1}^{n} z_{j}^{2}}\right),
$$

where $z_{i}=\hat{t}_{i}^{\prime}-\hat{t}_{i}$. Assuming $\hat{t}_{i} \frown N\left(t_{i}, v_{1}\right)$, and $\hat{t}_{i}^{\prime} \frown N\left(t_{i}, v_{2}\right)$ for $i=1,2, \ldots, n$, the $z_{i} \frown N(0, v)$, where $v=v_{1}+v_{2}$, 
then

$$
\begin{aligned}
& \operatorname{cov}\left(z_{i}, z_{j}\right)=E\left(z_{i} z_{j}\right)=E\left[\left(\hat{t}_{i}^{\prime}-t_{i}\right)-\left(\hat{t}_{i}-t_{i}\right)\right]\left[\left(\hat{t}_{j}^{\prime}-t_{j}\right)-\left(\hat{t}_{j}-t_{j}\right)\right] \\
& =E\left(\hat{t}_{i}^{\prime}-t_{i}\right)\left(\hat{t}_{j}^{\prime}-t_{j}\right)+E\left(\hat{t}_{i}-t_{i}\right)\left(\hat{t}_{j}-t_{j}\right)=\operatorname{cov}\left(\hat{t}_{i}^{\prime}, \hat{t}_{j}^{\prime}\right)+\operatorname{cov}\left(\hat{t}_{i}, \hat{t}_{j}\right) \\
& =c_{2}+c_{1}=c
\end{aligned}
$$

Now $z_{1}, z_{2}, \ldots, z_{n}$ have the multivariate normal distribution with mean vector $\underline{\mu}=\underline{0}$, and variance covariance matrix $\sum_{n \times n}$ with the element $\mathrm{V}$ along the diagonal and the element $\mathrm{C}$ otherwise. Now one can write the density function as:

$$
f\left(z_{1}, z_{2}, \ldots, z_{n}\right)=C t \cdot e^{-\frac{1}{2^{2}} \sum^{-1} \underline{z}} d \underline{z},-\infty<z_{i}<+\infty .
$$

This is an even function and the expectation of any odd function of $z_{i}$ 's in zero. Since $\frac{z_{i}}{\sum_{j=1}^{n} z_{j}^{2}}$ is an odd function, its expectation is zero. Going back to (7.3) one can write $E\left(\hat{\tau}_{i}\right)=t_{i}$. Hence $V\left(\hat{\tau}_{i}\right)=E\left[\left(\hat{t}_{i}-t_{i}\right)+\frac{\theta B s^{2}}{\sum_{j=1}^{n} z_{j}^{2}}\right]^{2}$,

$$
=E\left(\hat{t}_{i}-t_{i}\right)^{2}+\theta^{2} B^{2} E\left(s^{4}\right) \cdot E\left(\frac{z_{i}}{\sum_{j=1}^{n} z_{j}^{2}}+2 \theta B E\left(s^{2}\right) \cdot E \frac{z_{i}\left(\hat{t}_{i}-t_{i}\right)}{\sum_{j=1}^{n} z_{j}^{2}} .\right.
$$


Since

$$
s^{2} \cap \frac{\sigma^{2}}{f} X^{2}, E\left(s^{4}\right)=\frac{\sigma^{4}}{f^{2}} E\left(X^{2}\right)^{2}=\frac{f(f+2) \sigma^{4}}{f^{2}}=\frac{f+2}{f} \sigma^{4},
$$

(7.5) may be written as

$$
v\left(\hat{\tau}_{i}\right)=v_{1}+\frac{\theta^{2} B^{2}(f+2) \sigma^{4}}{f} \mathbf{E} \frac{z_{i}^{2}}{\left(\sum_{j=1}^{n} z_{j}^{2}\right)^{2}}+2 \theta B \sigma^{2} \mathbf{E} \frac{z_{i}\left(\hat{t}_{i}-t_{i}\right)}{\sum_{j=1}^{n} z_{j}^{2}} .
$$

By symmetry of the $z_{j}$ 's, we can write:

$$
\mathrm{E} \frac{\mathrm{z}_{1}^{2}}{\mathrm{z}_{1}^{2}+\mathrm{z}_{2}^{2}+\ldots+\mathrm{z}_{\mathrm{n}}^{2}}=\mathrm{E} \frac{\mathrm{z}_{2}^{2}}{\mathrm{z}_{1}^{2}+\mathrm{z}_{2}^{2}+\ldots+\mathrm{z}_{\mathrm{n}}^{2}}=\ldots=\mathrm{E} \frac{\mathrm{z}_{n}^{2}}{\mathrm{z}_{1}^{2}+\mathrm{z}_{2}^{2}+\ldots+\mathrm{z}_{n}^{2}},
$$

i.e.,

$$
E \frac{z_{i}^{2}}{\left(\sum_{j=1}^{n} z_{j}^{2}\right)^{2}}=\frac{1}{n} E \frac{\sum_{j=1}^{n} z_{j}^{2}}{\left(\sum_{j=1}^{n} z_{j}^{2}\right)^{2}}=\frac{1}{n} E \frac{1}{\sum_{j=1}^{n} z_{j}^{2}} .
$$

Now,

$$
\mathbb{E} \frac{z_{i}\left(\hat{t}_{i}-t_{i}\right)}{\sum_{j=1}^{n} z_{j}^{2}}=E_{z_{1}} \ldots z_{n}\left(\frac{z_{i}}{\sum_{j=1}^{n} z_{j}^{2}}\right) \cdot E_{\hat{t}_{i}} \mid z_{1}, z_{2}, \ldots, z_{n}\left(\hat{t}_{i}-t_{i}\right)
$$

Consider now the multivariate vector $\left[\hat{t}_{i}, z_{i}, z_{1}, z_{2}, \ldots, z_{i-1}, z_{i+1}, \ldots, z_{n}\right]$ with mean vector 
$\left[t_{i}, 0,0, \ldots, 0\right]$ and variance covariance matrix:

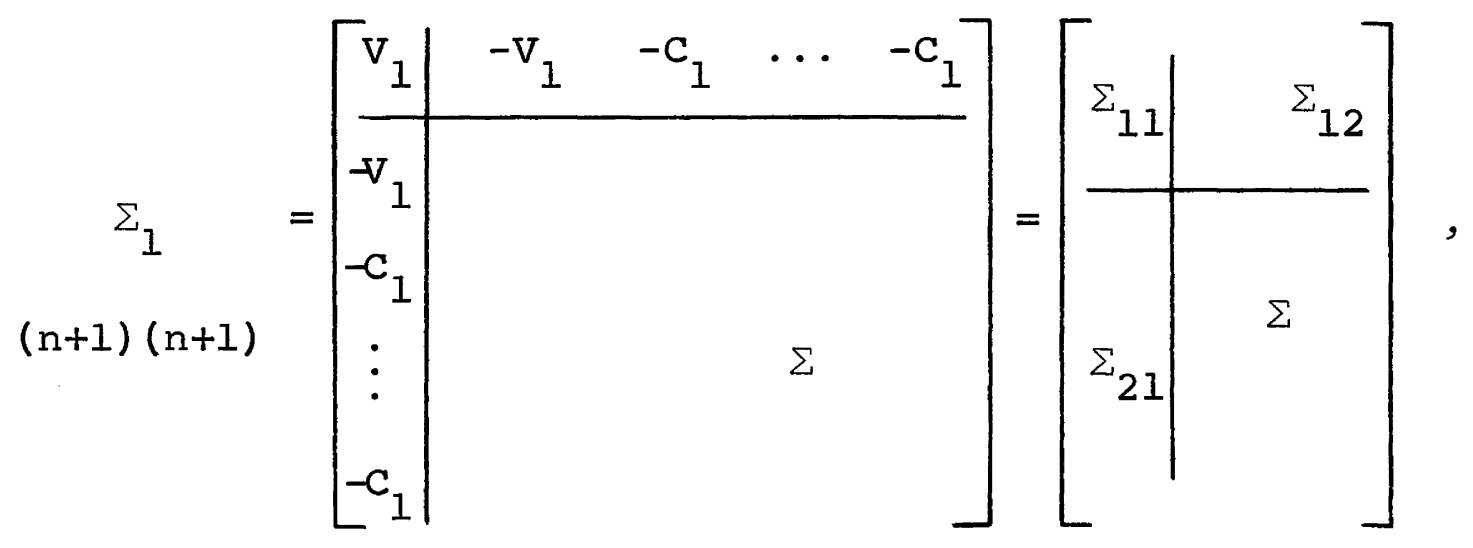

where $\Sigma$ is as defined before. Now

$$
E_{\hat{t}_{i}} \mid z_{1}, z_{2}, \ldots, z_{n}\left(\hat{t}_{i}\right)=t_{i}+\Sigma_{12} \Sigma^{-1} \underline{z} \text {, }
$$

where $\Sigma^{-1}$ is a two-element matrix with diagonal elements d and off-diagonal elements $f$, where:

$$
d=\frac{-[(n-2) C+V]}{C^{2}(n-1)-V[(n-2) C+V]},
$$

and

$$
f=\frac{C}{C^{2}(n-1)-V[(n-2) C+V]} .
$$

Now

$$
\begin{gathered}
\Sigma_{12} \Sigma^{-1}=\left(-v_{1},-c_{1},-c_{1}, \ldots,-c_{1}\right)\left[\begin{array}{ccccc}
d & f & f & \ldots & f \\
f & d & f & \ldots & f \\
\cdot & \cdot & \cdot & . & \cdot \\
f & f & \ldots & d
\end{array}\right]= \\
{\left[\left(-v_{1} d-(n-1) c_{1} f\right),\left(-c_{1} d-(n-2) c_{1} f-v_{1} f\right),\left(-c_{1} d-(n-2) c_{1} f-v_{1} f\right),\right.} \\
\ldots, \text { the same } \ldots . . .
\end{gathered}
$$


But

$$
\begin{aligned}
& -V_{1} d-(n-1) c_{1} f=\frac{V_{1}[(n-2) c+V]-(n-1) c_{1} c}{c^{2}(n-1)-V[(n-2) c+v]} \\
& =\frac{V_{1}(n-1) C-C_{1}(n-1) C+V_{1}(V-C)}{(n-1) c^{2}-(n-1) V C-V(V-C)}=\frac{V_{1}(V-C)-(n-I) C\left(V_{1}-c_{1}\right)}{(n-1) C(c-V)+V(-V+C)} \text {, } \\
& =\frac{(n-1) c\left(v_{1}-C_{1}\right)+V_{1} V-V_{1}\left(c_{1}+C_{2}\right)}{(n-1) c(c-V)+V(c-V)}, \quad\left(\text { since } c=c_{1}+c_{2}\right) \\
& =\frac{(n-1) C\left(V_{1}-C_{1}\right)+V_{1} V-V_{1} C_{1}-V_{2} C_{1}}{(c-V)[(n-1) C+V]}
\end{aligned}
$$

using (6.5). Thus

$$
\begin{aligned}
-v_{1} d-(n-1) c_{1} f & =\frac{(n-1) c\left(v_{1}-c_{1}\right)+v\left(v_{1}-c_{1}\right)}{(c-v)[(n-1) c+v]}, \quad\left(v=v_{1}+v_{2}\right) \\
& =\frac{v_{1}-C_{1}}{C-V} .
\end{aligned}
$$

To compute the remaining elements in the nxl row $\Sigma_{12} \Sigma^{-1}$, one needs to compute only

$$
\begin{aligned}
& -c_{1} d-(n-2) c_{1} f-v_{1} f=-c_{1}[d+(n-2) f]-V_{1} f \\
& =\frac{-C_{1}\{-[(n-2) C+V]+(n-2) C\}-V_{1} C}{(n-1) C^{2}-V[(n-2) C+V]} \text {, } \\
& =\frac{V c_{1}-V_{1} c}{(n-1) c^{2}-V[(n-2) c+V]}=\frac{V_{1} c_{1}+V_{2} c_{1}-V_{1} c_{1}-V_{1} c_{2}}{(n-1) c^{2}-V[(n-2) c+V]} \\
& =\frac{V_{2} C_{1}-V_{1} C_{2}}{(n-1) c^{2}-V[(n-2) c+V]}=0 \text {, }
\end{aligned}
$$


i.e.,

$$
-c_{1} d-(n-2) c_{1} f-V_{1} f=0
$$

Now (7.9) can be written

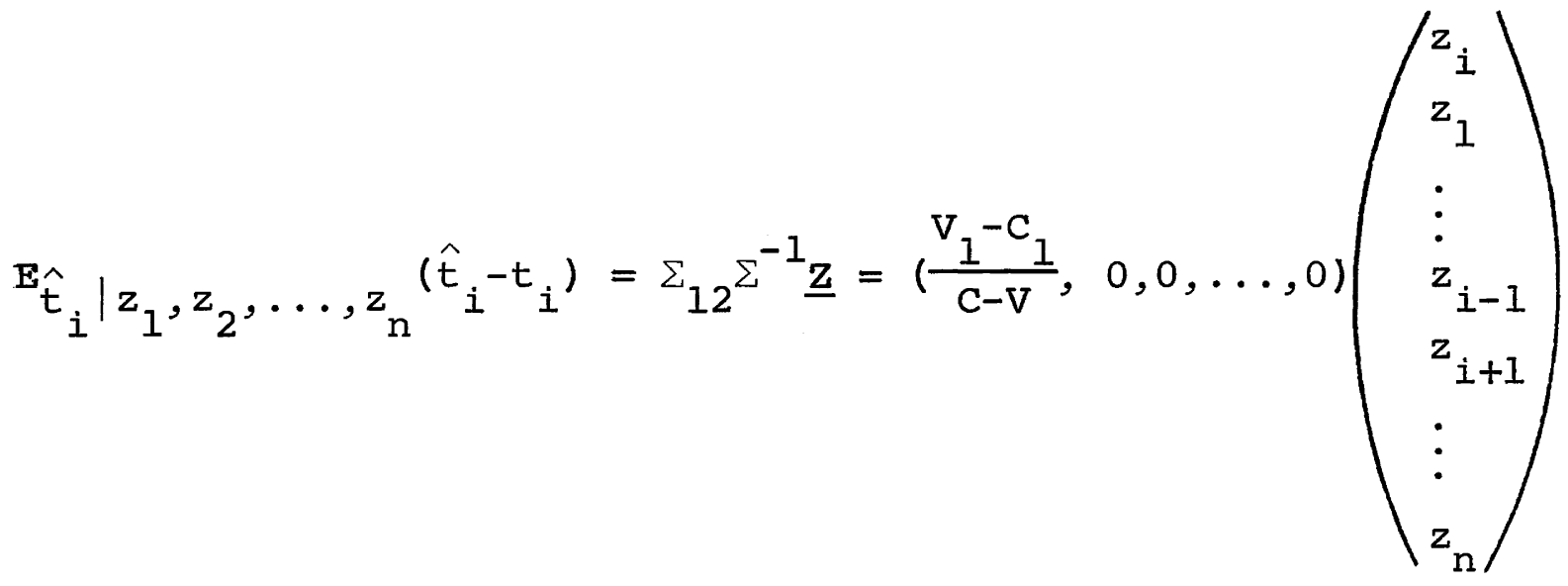

$$
\begin{aligned}
& =\frac{V_{1}-C_{1}}{C-V} z_{i}=-\frac{V_{1}-C_{1}}{V-C} z_{i} .
\end{aligned}
$$

Substituting $(7.7)$ and $(7.14)$ into (7.6),

$$
v\left(\hat{\tau}_{i}\right)=v_{1}+\frac{v_{1}^{2} B^{2}(f+2)}{n f} E \frac{1}{\sum_{j=1}^{n} z_{j}^{2}}-2 v_{1} B E\left[\frac{v_{1}-C_{1}}{v-C} \cdot \frac{z_{i}^{2}}{\sum_{j=1}^{n} z_{j}^{2}}\right]
$$

It should be noted that $\theta \sigma^{2}=v_{1}$. Utilizing again the symmetry property, $\quad \mathbf{E} \frac{z_{i}^{2}}{\sum_{j=1}^{n} z_{j}^{2}}=\frac{1}{n}$, hence: 


$$
V\left(\hat{\tau}_{i}\right)=v_{1}+\frac{v_{1}^{2} B^{2}(f+2)}{n f} E \frac{1}{\sum_{j=1}^{n} z_{j}^{2}}-2 \frac{v_{1} B\left(v_{1}-C_{1}\right)}{n(V-C)} .
$$

Using (5.7) with $\mathrm{a}=\mathrm{v}, \mathrm{b}=\mathrm{C}$,

$$
E \frac{1}{\sum_{j=1}^{n} z_{j}^{2}}<\frac{1}{(n-3)(v-C)}
$$

Now one can write

$$
V\left(\hat{\tau}_{i}\right)<v_{1}+\frac{V_{1}^{2} B^{2}(f+2)}{n f(n-3)(V-C)}-2 \frac{v_{1} B\left(v_{1}-C_{1}\right)}{n(V-C)} .
$$

we would like the quantity $\frac{v_{1}^{2} B^{2}(f+2)}{n(n-3) f(V-C)}-2 \frac{v_{1} B\left(v_{1}-C_{1}\right)}{n(v-c)}$ to be negative. First we notice by $(6.8)$ that $\mathrm{v}-\mathrm{C}=-\mathrm{vC}=-\mathrm{v}\left(\mathrm{C}_{1}+\mathrm{C}_{2}\right)=\frac{\mathrm{k}}{\mathrm{Av}} \sigma^{2}+\frac{\mathrm{k}}{\mathrm{r}-\lambda} \sigma^{\prime 2}$ which is always positive; if, in addition $n>3$, then the above quadratic in $B$ is negative whenever

$$
0<B<\frac{2 f\left(v_{1}-C_{1}\right)(n-3)}{v_{1}(f+2)} .
$$

The value of $B$ which makes it the most negative is

$$
B=\frac{f\left(v_{1}-C_{1}\right)(n-3)}{V_{1}(f+2)} .
$$


From (6.9)

$$
B=\frac{f v(n-3)}{(v-1)(f+2)}
$$

Now substituting $B$ in $(7.18)$,

$$
\begin{gathered}
v\left(\hat{\tau}_{i}\right)<v_{1}+\frac{V_{1}^{2}}{V-C}\left[\frac{f+2}{n f(n-3)} \frac{v^{2} f^{2}(n-3)^{2}}{(v-1)^{2}(f+2)^{2}}-\frac{2 v}{n(v-1)} \cdot \frac{v f(n-3)}{(v-1)(f+2)^{2}}\right] \\
=v_{1}+\frac{v_{1}^{2}}{V-C}\left[\frac{-v^{2} f(n-3)}{n(v-1)^{2}(f+2)}\right],
\end{gathered}
$$

i.e.,

$$
v\left(\hat{\tau}_{i}\right)<v_{1}-\frac{v^{2} f(n-3)}{n(v-1)^{2}(f+2)} \frac{v_{1}^{2}}{V-C}
$$

It is obvious that one should take $\mathrm{n}$ as large as possible, but since $\mathrm{n}$ is strictly less than $\mathrm{v}$, take $\mathrm{n}=\mathrm{v}-1$. It should be noted that one cannot take $\mathrm{n}=\mathrm{v}$, for then the matrix $\Sigma$ is singular. Hence

$$
\mathrm{v}\left(\hat{\tau}_{i}\right)<v_{1}-\frac{v_{i}^{2} f(v-4)}{(v-1)^{3}(f+2)} \frac{v_{1}^{2}}{v-C}
$$

As was mentioned previously, the quantity $\frac{\mathrm{v}_{1}^{2}}{\mathrm{v}_{1}+\mathrm{v}_{2}}$ represents the utmost improvement possible over the intra-block variance. Now one can write $\frac{V_{1}^{2}}{V-C}=\frac{V_{1}^{2}}{v_{1}+v_{2}}+\frac{V_{1}^{2} C}{V(V-C)}$ $=\frac{\mathrm{V}_{1}^{2}}{\mathrm{~V}}\left[1+\frac{\mathrm{C}}{\mathrm{V}-\mathrm{C}}\right]=\frac{\mathrm{v}_{1}^{2}}{\mathrm{~V}}\left(1+\frac{\mathrm{C}}{-\mathrm{vC}}\right)$, using $(6.8)$. 
Then $\frac{\mathrm{V}_{1}^{2}}{\mathrm{~V}-\mathrm{C}}=\frac{\mathrm{V}_{1}^{2}}{\mathrm{v}_{1}+\mathrm{V}_{2}} \frac{\mathrm{v}-1}{\mathrm{v}}$, and substituting into $(7.21)$,

$$
\mathrm{v}\left(\hat{\tau}_{i}\right)<\mathrm{v}_{1}-\frac{\mathrm{fv}(\mathrm{v}-4)}{(\mathrm{v}-1)^{2}(\mathrm{f}+2)} \cdot \frac{\mathrm{v}_{1}^{2}}{\mathrm{v}_{1}+\mathrm{v}_{2}} .
$$

The upper bound of the variance of the proposed estimate is uniformly better than the intra-block variance for any B.I.B. design when $v>4$. Substituting $n=v-1$ into (7.19) one obtains $B=\frac{f v(v-4)}{(f+2)(v-1)}$, and then from (7.2),

$$
\hat{\tau}_{i}=\hat{t}_{i}+\frac{\theta v(v-4) f S^{2}}{(f+2)(v-1) \sum_{j=1}^{-1}\left(\hat{t}_{j}^{\prime}-\hat{t}_{j}\right)^{2}}\left(\hat{t}_{i}-\hat{t}_{i}\right) \text {, }
$$

and recalling that $\theta=\frac{k(v-1)}{\lambda v^{2}}$ or $\frac{\theta v}{v-1}=\frac{k}{\lambda v}$,

$$
\hat{\tau}_{i}=\hat{t}_{i}+\frac{k(v-4) s^{2}}{\lambda v(t+2) \sum_{j=1}^{v-1}\left(\hat{t}_{j}^{\prime}-\hat{t}_{j}\right)^{2}}\left(\hat{t}_{i}-\hat{t}_{i}\right),
$$

where $\mathrm{S}^{2}=f \mathrm{~S}^{2}$ is the error sum of squares in the intraanalysis. From (7.22) it is seen that the ratio of improvement or the recovery ratio is:

$$
D_{1}=\frac{f v(v-4)}{(v-1)^{2}(f+2)}
$$




\subsection{Exact Results for Theorem 2}

Using lemma 2 one can get a fairly exact value for $\mathrm{E} \frac{\mathrm{l}}{\sum \mathrm{z}_{\mathrm{i}}^{2}}$. Here $\mathrm{a}=\mathrm{v}, \mathrm{b}=\mathrm{c}, \mathrm{n}=\mathrm{v}-1$, so,

$$
E\left(\frac{1}{\sum_{i=1}^{v-1} z_{i}^{2}}\right)=\frac{\Gamma\left(\frac{v-3}{2}\right)}{\Gamma\left(\frac{v-1}{2}\right) \Gamma\left(\frac{1}{2}\right)} \int_{0}^{1} \frac{u^{\frac{v-4}{2}}(1-u)^{-\frac{1}{2}}}{2(v-1) C(1-u)+2(v-C)} d u,
$$

but since $\mathrm{V}-\mathrm{C}=-\mathrm{vC}$, one obtains

$$
\begin{aligned}
E\left(\frac{1}{v-1}\right) & =\frac{2}{(v-3) \beta\left(\frac{v-2}{2}, \frac{1}{2}\right)} \int_{0}^{1} \frac{u^{\frac{v-4}{2}}(1-u)^{-\frac{1}{2}}}{2(v-1) C(1-u)-2 v C} d u \\
& =\frac{1}{-C(v-3) \beta\left(\frac{v-2}{2}, \frac{1}{2}\right)} \int_{0}^{1} \frac{u^{\frac{v-4}{2}}(1-u)^{-\frac{1}{2}}}{1+(v-1) u} d u
\end{aligned}
$$

The problem now is to evaluate the quantity,

$$
F=\frac{1}{\beta\left(\frac{v-2}{2}, \frac{1}{2}\right)} \int_{0}^{1} \frac{u^{\frac{v-4}{2}}(1-u)^{-\frac{1}{2}}}{1+(v-1) u} d u,
$$

for different values of $v$. Later a method of computing this quantity will be given. Now, one may write

$$
E\left(\frac{1}{\sum_{i=1}^{-1}}\right)=\frac{-F}{C(v-3)}
$$


Substituting (7.1.1) into (7.16), and taking $n=v-1$, one obtains

$$
V\left(\hat{\tau}_{i}\right)=v_{1}+\frac{v_{1}^{2} B^{2}(f+2) F}{-C f(v-1)(v-3)}-\frac{2 V_{1} B\left(V_{1}-C_{1}\right)}{(v-1)(v-C)},
$$

but $\mathrm{V}-\mathrm{C}=-\mathrm{vc}$ and $\frac{\mathrm{v}_{1}-\mathrm{C}_{1}}{\mathrm{v}}=\frac{\mathrm{v}_{1}}{\mathrm{v}-1}$ or $\mathrm{v}_{1}-\mathrm{C}_{1}=\frac{\mathrm{v} \mathrm{v}_{1}}{\mathrm{v}-1}, \frac{\mathrm{v}_{1}-\mathrm{C}_{1}}{\mathrm{v}-\mathrm{C}}$ $=\frac{v_{1}}{-C(v-1)}$

$$
V\left(\hat{\tau}_{i}\right)=v_{1}+\frac{v_{1}^{2} B}{-(v-1) C}\left[\frac{B(f+2) F}{f(v-3)}-\frac{2}{v-1}\right] .
$$

Since $\mathrm{C}$ is negative, one needs

$$
0<B<\frac{2 f(v-3)}{F(v-1)(f+2)} .
$$

The required $B$ is

$$
B=\frac{f(v-3)}{F(v-1)(f+2)} .
$$

Substituting (7.1.3) into (7.1.2), one may write

$$
v\left(\hat{\tau}_{i}\right)=v_{1}+\frac{v_{1}^{2} B}{-C(v-1)}\left[\frac{-1}{v-1}\right]=v_{1}-\frac{v_{1}^{2} B}{-C(v-1)^{2}},(7.1 .4)
$$

since $\frac{v_{1}^{2}}{V-C}=\frac{v_{1}^{2}}{-v C}=\frac{v_{1}^{2}}{v_{1}+v_{2}} \cdot \frac{v-1}{v}$, then (7.1.4) becomes

$$
v\left(\hat{\tau}_{i}\right)=v_{1}-\frac{B}{v-1} \frac{v_{1}^{2}}{v_{1}+v_{2}}
$$

The combined estimate in (7.2) becomes, after substituting, 
the value of $\theta$ :

$$
\begin{aligned}
\hat{\tau}_{i} & =\hat{t}_{i}+\frac{\theta B S^{2}}{\sum_{j=1}^{v-1}\left(\hat{t}_{j}^{\prime}-\hat{t}_{j}\right)^{2}}\left(\hat{t}_{i}^{\prime}-\hat{t}_{i}\right), \\
& =\hat{t}_{i}+\frac{k(v-1) B s^{2}}{\lambda v^{2} \sum_{j=1}^{v-1}\left(\hat{t}_{j}^{\prime}-\hat{t}_{j}\right)^{2}}\left(\hat{t}_{i}^{\prime}-\hat{t}_{i}\right) .
\end{aligned}
$$

From (7.1.5) the recovery ratio is

$$
D_{2}=B /(v-1)
$$

The estimate in $(7.1 .6)$ can be applied whenever $v>3$. The values of $B$ and $D_{2}$ appear in Table II for the 58 B.I.B. designs listed in Reference (2).

\subsection{1* Method for computing $F$}

The hypergeometric series,

$$
F(a, b ; c ; z)=\sum_{n=0}^{\infty} \frac{(a)_{n}(b)_{n}}{n !(c)_{n}} z^{n} \text {, }
$$

converges to the definite integral [see Reference (12)]:

$$
I=\frac{1}{\beta(b, c-b)} \int_{0}^{1} u^{b-1}(1-u)^{c-b-1}(1-u z)^{-a} d u
$$

*I am indebted to Dr. I. R. Shenton who introduced me to this method. 
Now one may write:

$$
F=\frac{1}{\beta\left(\frac{v-2}{2}, \frac{1}{2}\right)} \int_{0}^{l} \frac{u^{\frac{v-4}{2}}(1-u)^{-\frac{1}{2}}}{1+(v-1) u} d u .
$$

Comparing (7.1.9) and (7.1.10), we get:

$$
\begin{aligned}
& \mathrm{b}-1=\frac{\mathrm{v}-4}{2}, \quad \mathrm{c}-\mathrm{b}-1=-\frac{1}{2}, \quad \mathrm{z}=1-\mathrm{v}, \quad \mathrm{a}=1, \\
& \text { or } \quad \mathrm{b}=\frac{\mathrm{v}-2}{2}, \quad \mathrm{c}=\frac{\mathrm{v}-1}{2}, \quad \mathrm{z}=1-\mathrm{v}, \quad \mathrm{a}=1 .
\end{aligned}
$$

One needs to evaluate, therefore, the quantity,

$$
F\left(1, \frac{v-2}{2} ; \frac{v-1}{2} ; 1-v\right)=F\left(\frac{v-2}{2}, 1 ; \frac{v-1}{2} ; 1-v\right),
$$

due to the symmetry of $a$ and $b$ in the hypergeometric form above. Now $F(b, 1 ; c ; z)$ can be put in the form of Gauss's continued fraction as follows [see Reference (13)]:

$$
F(b, 1 ; C ;
$$$$
\text { z) = }
$$$$
=\frac{1}{1-\frac{h_{1} z}{1-\frac{\left(1-h_{1}\right) h_{2} z}{\left(1-h_{2}\right) h_{3} z}}},
$$

$$
1-
$$

where $h_{2 p-1}=\frac{b+p-1}{c+2 p-2}, \quad h_{2 p}=\frac{p}{c+2 p-1}, \quad p=1,2,3, \ldots$. 
The convergence is very rapid and it is far easier to compute than the usual simpson rule, in this case. It is to be noted that the terms required to assure an accuracy up to the sixth decimal place, range from 4 terms for $\mathrm{v}=91$ to 14 terms for $\mathrm{v}=4$.

7.2 Application of Theorem 1 in B.I.B. Designs

Consider the vxv matrix

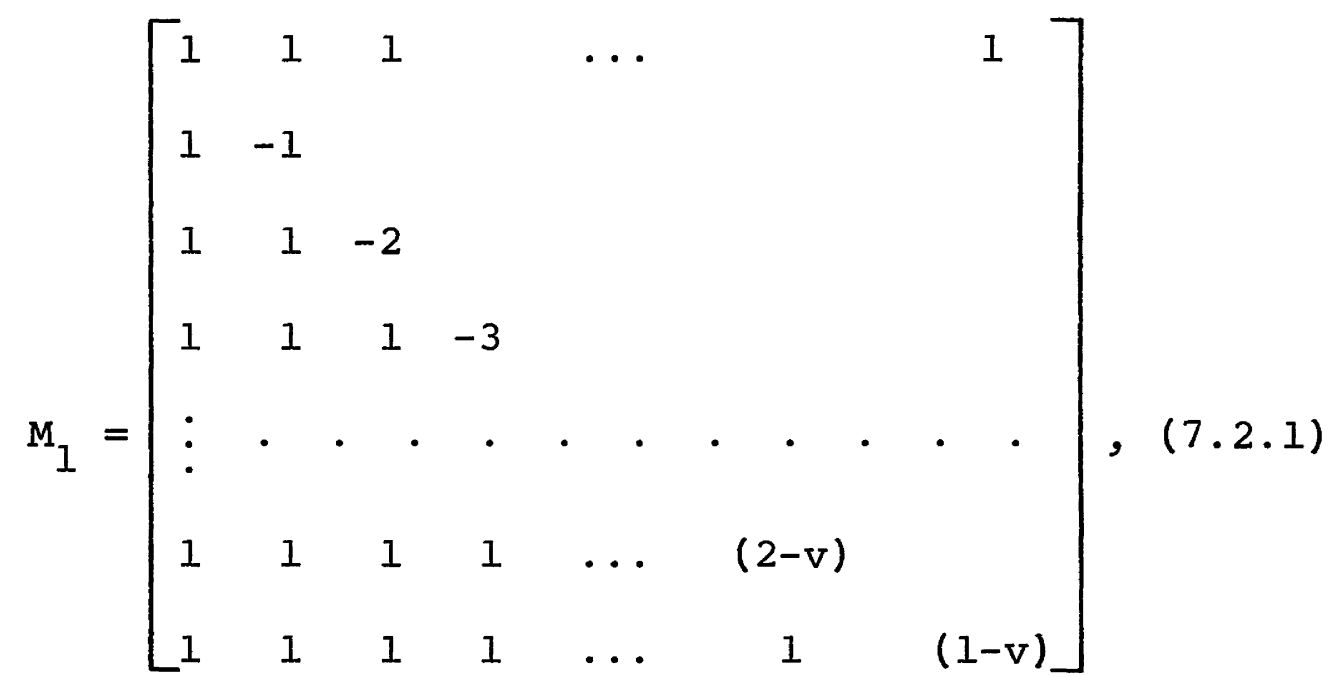

and let $M$ be the orthogonal matrix we get by normalizing $M_{1}$. Consider the two vectors of comparisons:

$$
\underline{U}=\left[\begin{array}{c}
0 \\
U_{1} \\
U_{2} \\
\vdots \\
U_{v-1}
\end{array}\right]=M\left[\begin{array}{c}
\hat{t}_{1} \\
\hat{t}_{2} \\
\vdots \\
\hat{t}_{v}
\end{array}\right]=M \underline{\hat{t}},
$$


and

$$
\underline{x}=\left[\begin{array}{c}
0 \\
x_{1} \\
x_{2} \\
\vdots \\
x_{v-1}
\end{array}\right]=m\left[\begin{array}{c}
\hat{t}_{1}^{\prime} \\
\hat{t}_{2}^{\prime} \\
\vdots \\
\hat{t}_{v}^{\prime}
\end{array}\right]=m \underline{\hat{t}}^{\prime} .
$$

In general,

$$
\begin{aligned}
& v\left(U_{m}\right)=v\left[\frac{1}{\sqrt{m(m+1)}}\left(\hat{t}_{1}+\hat{t}_{2}+\ldots+\hat{t}_{m}-m \hat{t}_{m+1}\right)\right] \\
& =\frac{1}{m(m+1)}\left[m(m+1) v_{1}+m(m-1) c_{1}-2 m\left(m c_{1}\right)\right]=v_{1}-c_{1}
\end{aligned}
$$

This result is independent of $\mathrm{m}$, that is, all $U_{i} ' s$, where $i=1,2, \ldots, v-1$, have the same variance, namely $v_{1}-C_{1}$. Similarly, $v\left(x_{i}\right)=V_{1}^{\prime}-c_{1}^{\prime}$ for $i=1,2, \ldots, v-1$. Therefore, theorem 1 is applicable for combining the two comparisons $\mathrm{U}_{i}$ and $\mathrm{x}_{i}$, and their combined estimate is:

$$
\hat{\tau}_{i}=U_{i}+\frac{\theta(v-3) s^{2}}{\sum_{j=1}^{v-1}\left(x_{j}-U_{j}\right)^{2}}\left(x_{i}-U_{i}\right) \quad, \quad\left(\theta=\frac{\rho f}{f+2}\right)
$$

but $v_{1}-c_{1}=\frac{k}{\lambda v} \sigma^{2}$, hence $\rho=\frac{k}{\lambda v}$, and

$$
\hat{\tau}_{i}=u_{i}+\frac{f k(v-3) s^{2}}{(f+2) \lambda v \sum_{j=1}^{v-1}\left(x_{j}-U_{j}\right)^{2}}\left(x_{i}-U_{i}\right) .
$$


It should be noted that

$$
\begin{aligned}
& \sum_{j=1}^{v-1}\left(x_{j}-U_{j}\right)^{2}=(\underline{x}-\underline{u})^{\prime}(\underline{x}-\underline{u})=(\underline{\hat{t}} \cdot-\underline{\hat{t}})^{\prime} M^{\prime} M(\underline{\hat{t}} \cdot-\underline{\hat{t}}) \\
& =\left(\underline{\hat{t}}^{\prime}-\underline{\hat{t}}\right)^{\prime}\left(\underline{\hat{t}}^{\prime}-\hat{\underline{t}}\right)=\sum_{j=1}^{\mathrm{V}}\left(\hat{t}_{j}^{\prime}-\hat{t}_{j}\right)^{2} . \quad \text { Let } \hat{\underline{\tau}}=\left[\begin{array}{l}
\hat{\tau}_{1} \\
\hat{\tau}_{2} \\
\vdots \\
\hat{\tau}_{v-1}
\end{array}\right]=M\left[\begin{array}{c}
T_{1} \\
T_{2} \\
\vdots \\
T_{v-1}
\end{array}\right]=M \underline{T},
\end{aligned}
$$

then (7.2.5) can be written in vector notation as

$$
M \underline{T}=\frac{\hat{t}}{(f+2) \lambda v \sum_{j=1}^{V}\left(\hat{t}_{j}^{\prime}-\hat{t}_{j}\right)^{2}} M\left(\hat{t}^{\prime}-\hat{t}\right) \text {. }
$$

Since $M^{\prime} M=I$, one can write

$$
T_{i}=\hat{t}_{i}+\frac{f k(v-3) s^{2}}{(f+2) \lambda v \sum_{j=1}^{v}\left(\hat{t}_{j}-\hat{t}_{j}\right)^{2}}\left(\hat{t}_{i}-\hat{t}_{i}\right), \quad i=1,2,3 \ldots v .
$$

This is applicable when $v>3$, and the recovery ratio here is

$$
D_{3}=\frac{(v-3) f}{(v-1)(f+2)}
$$

The results for $D_{1}$ as given in $(7.2 .4)$, and $D_{3}$ above appear in Table II. The results show that $\mathrm{D}_{2}$ and $\mathrm{D}_{3}$ are always better than $\mathrm{D}_{1}$; the three ratios approach practically the same value as $v$ becomes relatively large. $D_{2}$ and $D_{3}$ 
are practically the same, but $\mathrm{D}_{3}$ is,

, always better than $D_{2}$.

7.3 Procedure for Recovery of Inter-block Information in

B.I.B. Designs

Compute:

(1) $\mathrm{Y}_{i .}=\sum_{j=1}^{b} \delta_{i j} \mathrm{y}_{i j}, \quad i=1,2,3 \ldots v \cdot \quad$ (Total yield of i-th treatment).

(2) $Y_{\cdot j}=\sum_{i=1}^{V} \delta_{i j} Y_{i j}, j=1,2, \ldots, b . \quad$ (Total yield of j-th block.)

(3) $Y_{.}=\sum_{i j} \delta_{i j} y_{i j} \cdot \quad$ (Grand total.)

(4) $Q_{i}=Y_{i}$. $-\frac{1}{k}$ (Sum of block totals in which treatment i occurs.),

$i=1,2, \ldots, v . \quad$ (Adjusted treatment totals.)

(5) $\hat{t}_{i}=\frac{Q_{i}}{r E}, \quad i=1,2, \ldots, v . \quad$ (The intra-estimate,

$$
E=\frac{(k-1) v}{k(v-1)}
$$

(6) $\operatorname{SST}=\sum_{i=1}^{V} \hat{t}_{i} \mathbf{Q}_{i} \cdot$ (Treatment sum of squares.) 
(7) $S^{2}=\frac{I}{f}\left(\sum_{i j} \delta_{i j} \mathrm{y}_{i j}^{2}-S S T-\frac{1}{k} \sum_{j=1}^{b} Y_{j}^{2}\right) \cdot \quad$ (Error mean square, $f=b k-b-v+l$.

(8) $Q_{i}+Q_{i}^{\prime}=Y_{i \cdot}-\frac{I}{V} Y \ldots, \quad i=I, 2, \ldots, v \cdot$ (Sum of intra- and inter-adjusted totals.)

(9) $\hat{t}_{i}^{\prime}-\hat{t}_{i}=\frac{Q_{i}}{r(I-E)}-\frac{Q_{i}}{r E}=\frac{E\left(Q_{i}+Q_{i}\right)-Q_{i}}{r E(I-E)}, i=1,2, \ldots, v$.

(10) $\sum_{i=1}^{y}\left(\hat{t}_{i}-\hat{t}_{i}\right)^{2}$

(II) $J=\frac{f k(v-3) s^{2}}{(f+2) \lambda v \sum_{i=1}\left(\hat{t}_{i}^{i}-\hat{t}_{i}\right)^{2}}$

(12) The combined estimate $T_{i}=\hat{t}_{i}+J\left(\hat{t}_{i}-\hat{t}_{i}\right)$,

$$
i=1,2, \ldots, v \text {. }
$$

This estimate recovers the ratio $D_{3}$ of the utmost possible recovery, and $D_{3}$ appears in Table II. 
7.4 Worked Example

An experiment with 6 treatments in blocks of 2 will be worked out. The data is taken from Reference (2), page 444; the treatment estimates obtained in (2) by applying the 
conventional method are to be compared with the results of the new method.

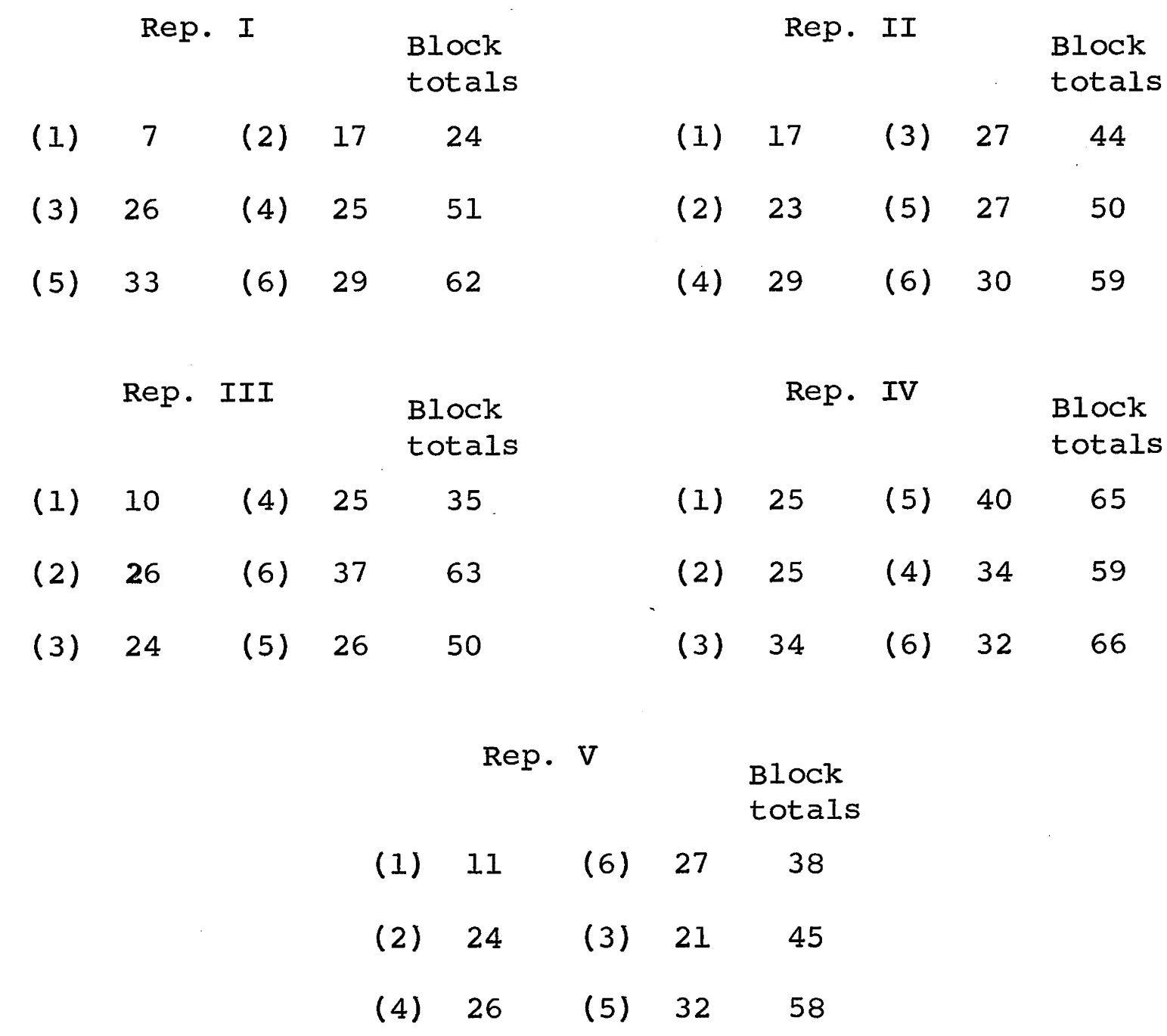

In this experiment $\mathrm{v}=6, \mathrm{k}=2, \mathrm{r}=5, \mathrm{~b}=15, \lambda=1, \mathbf{E}=.6$. 
The following table is to be set up.

$\begin{array}{crrrrrrr}\begin{array}{c}\text { Treat. } \\ \text { No. }\end{array} & Y_{i .} & Q_{i} & \hat{t}_{i} & Q_{i}+Q_{i}^{\prime} & \hat{t}_{i}^{\prime}-\hat{t}_{i} & \hat{\tau}_{i} & T_{i} \\ 1 & 70 & -33.0 & -11.00 & -58.17 & -1.59 & -11.31 & -11.2 \\ 2 & 115 & -5.5 & -1.83 & -13.17 & -2.00 & -2.23 & -2.1 \\ 3 & 132 & 4.0 & 1.33 & 3.83 & -1.42 & 1.05 & 1.1 \\ 4 & 139 & 8.0 & 2.67 & 10.83 & -1.25 & 2.42 & 2.5 \\ 5 & 158 & 15.5 & 5.17 & 29.83 & 2.00 & 5.57 & 5.5 \\ 6 & 155 & 11.0 & 3.67 & 26.83 & \frac{4.25}{4.52} & 4.4 \\ & & 0 & 0 & 0 & 0 & 0 & \end{array}$

For Treatment 1, for instance, the following steps should be carried out.

Step 1: Treatment total $=\mathrm{Y}_{1}=7+17+\ldots+11=70$. Step 2: $Q_{1}=Y_{1 i}-\frac{1}{2}$ (sum of block totals in which Treatment 1 appears), $=70-\frac{1}{2}(24+44+\ldots+38)=-33$.

Step 3: $\quad \hat{t}_{1}=\frac{Q_{1}}{r E}=\frac{-33}{5(.6)}=-11$,

where $\mathrm{E}=$ Efficiency of the design $=\frac{(\mathrm{k}-1) \mathrm{v}}{\mathrm{k}(\mathrm{v}-1)}=\frac{(2-1) \cdot 6}{2 \cdot 5}=.6$.

Step 4: $Q_{1}+Q_{1}^{\prime}=Y_{1} .-\frac{1}{v}(Y,)=.70-\frac{769}{6}=-58.17$, where $\mathrm{Y}_{\ldots}$ = Grand Total $=24+51+\ldots+58=769$. 
Step 5: $\hat{t}_{1}^{\prime}-\hat{t}_{1}=\frac{Q_{1}^{\prime}}{r(1-E)}-\frac{Q_{1}}{r E}=\frac{E\left(Q_{1}+Q_{1}^{\prime}\right)-Q_{1}}{r E(1-E)}$,

$$
=\frac{.6(-58.17)+33}{5(.6)(.4)}=-1.59 \text {. }
$$

After computing the same quantities for all treatments, one should compute the following quantities:

Sum squares of blocks (unadjusted)

$$
\begin{aligned}
& =\frac{1}{2}\left(24^{2}+51^{2}+\ldots+58^{2}\right)-\frac{(769)^{2}}{30}, \\
& =1051.5 .
\end{aligned}
$$

Sum squares of treatments (adjusted)

$$
\begin{aligned}
& =\sum_{i=1}^{v} \hat{t}_{i} \mathbf{Q}_{i}=\hat{t}_{1} \mathbf{Q}_{1}+\ldots+\hat{t}_{6} \mathbf{Q}_{6}, \\
& =520.2 .
\end{aligned}
$$

Error mean square $=\frac{1}{r v-v-b+1}($ Total sum of squares - Sum squares of treatments adjusted - sum squares of blocks unadjusted),

$=\frac{1}{10}(1649-520.2-1051.5)=7.73$.

$$
\begin{aligned}
\sum_{i=1}^{V}\left(\hat{t}_{i}^{\prime}-\hat{t}_{i}\right)^{2} & =\left(\hat{t}_{1}^{\prime}-\hat{t}_{1}\right)^{2}+\left(\hat{t}_{2}^{\prime}-\hat{t}_{2}\right)^{2}+\ldots+\left(\hat{t}_{6}^{\prime}-\hat{t}_{6}\right)^{2} \\
& =(-1.59)^{2}+(-2)^{2}+\ldots+(4.25)^{2}=32.2 .
\end{aligned}
$$




$$
J=\frac{f k(v-3) s^{2}}{(f+2) \lambda v \sum_{i=1}^{v}\left(t_{i}-t_{i}\right)^{2}}=\frac{10 \cdot 2 \cdot 3 \cdot(7 \cdot 73)}{12 \cdot 1 \cdot 6 \cdot(32 \cdot 2)}=
$$

now the combined estimate $\tau_{i}$ is:

$$
\hat{\tau}_{i}=\hat{t}_{i}+J\left(\hat{t}_{i}^{p}-\hat{t}_{i}\right)
$$

For the first treatment, for instance:

$$
\hat{\tau}_{1}=-11+(.20005)(-1.59)=-11.31
$$




\section{P.B.I.B. WITH TWO ASSOCIATE CLASSES}

\subsection{Definitions and Useful Relations}

An incomplete block design is said to be partially balanced with two associate classes if it satisfies the following requirements:

(i) The experimental material is divided into b blocks of $\mathrm{k}$ units each, different treatments being applied to the units in the same block.

(ii) There are $v(>k)$ treatments each of which occurs in $r$ blocks.

(iii) There can be established a relation of association between any two treatments satisfying the following requirements:

(a) Two treatments are either first associates or second associates.

(b) Each treatment has exactly $\mathrm{n}_{i}$ i-th associates $(i=1,2)$.

(c) Given any two treatments, which are i-th associates, the number of treatments common to the $j$-th associate of the first and the $k$-th associate of the second is $p_{j k}^{i}$ and is independent of the pair of treatments we start with. Also $p_{j k}^{i}=p_{k j}^{i} \quad(i, j, k=1,2)$. 
(iv) Two treatments which are i-th associates occur together in exactly $\lambda_{i}$ blocks $(i=1,2)$.

The numbers $v, r, k, b, n_{1}, n_{2}, \lambda_{1}$, and $\lambda_{2}$ are called parameters of the first kind, whereas the numbers $p_{j k}^{i}$ $(i, j, k=1,2)$ are called the parameters of the second kind.

The following relations between the parameters are known to hold:

$$
\begin{array}{ll}
\mathrm{vr}=\mathrm{bk}, & \mathrm{n}_{1}+\mathrm{n}_{2}=\mathrm{v}-1, \\
\mathrm{n}_{1} \lambda_{1}+\mathrm{n}_{2} \lambda_{2}=\mathrm{r}(\mathrm{k}-1), & \mathrm{p}_{11}^{1}+\mathrm{p}_{12}^{1}=\mathrm{n}_{1}-1, \\
\mathrm{p}_{21}^{1}+\mathrm{p}_{22}^{1}=\mathrm{n}_{2}, & \mathrm{p}_{11}^{2}+\mathrm{p}_{12}^{2}=\mathrm{n}_{1}, \\
\mathrm{p}_{21}^{2}+\mathrm{p}_{22}^{2}=\mathrm{n}_{2}-1, & \mathrm{n}_{1} \mathrm{p}_{12}^{1}=\mathrm{n}_{2} \mathrm{p}_{11}^{2}, \\
\mathrm{n}_{1} \mathrm{p}_{22}^{1}=\mathrm{n}_{2} \mathrm{p}_{12}^{2}, &
\end{array}
$$

In the analysis of such designs, $T_{i}$ is defined as the total of the observations for the i-th treatment, $B_{j}$ as the sum of the $k$ observations from the j-th block. $Q_{i}$ denotes the adjusted yield for the i-th treatment obtained by subtracting from $T_{i}$, the sum of the block averages for those blocks in which the $i$-th treatment occurs. Also, $s_{1}\left(Q_{i}\right)$ denotes the sum of the adjusted yields for all the first associates of the $i-t h$ treatment, and $G$ denotes the total of all $\mathbf{N}$ observations. 
R. C. Bose defines the constants $\Delta, H, c_{1}, c_{2}$ by the relations:

$$
\begin{aligned}
& \mathrm{K}^{2} \Delta=\left(\mathrm{a}+\lambda_{1}\right)\left(\mathrm{a}+\lambda_{2}\right)+\left(\lambda_{1}-\lambda_{2}\right)\left[\mathrm{a}(\mathrm{f}-\mathrm{g})+\mathrm{f} \lambda_{2}-g \lambda_{1}\right], \\
& \mathrm{KH}=\left(2 \mathrm{a}+\lambda_{1}+\lambda_{2}\right)+(f-g)\left(\lambda_{1}-\lambda_{2}\right), \\
& \mathrm{K} \Delta \mathrm{c}_{1}=\lambda_{1}\left(\mathrm{a}+\lambda_{2}\right)+\left(\lambda_{1}-\lambda_{2}\right)\left(f \lambda_{2}-g \lambda_{1}\right), \\
& \mathrm{K} \Delta \mathrm{c}_{2}=\lambda_{2}\left(\mathrm{a}+\lambda_{1}\right)+\left(\lambda_{1}-\lambda_{2}\right)\left(\mathrm{f} \lambda_{2}-g \lambda_{1}\right),
\end{aligned}
$$

where,

$$
a=r(k-1), \quad f=p_{12}^{1}, \quad g=p_{12}^{2} .
$$

In the intra-block analysis, the best linear estimate $\hat{t}_{i}$ of the treatment effect $t_{i}$ is given by,

$$
\hat{t}_{i}=\frac{k-c_{2}}{a} Q_{i}+\frac{c_{1}-c_{2}}{a} s_{1}\left(Q_{i}\right) \text {. }
$$

The variance of the intra-estimate of the difference between two treatment effects is given by:

$$
v\left(\hat{t}_{i}-\hat{t}_{u}\right)=\frac{2\left(k-c_{j}\right)}{a} \sigma^{2},
$$

where the treatments $i$ and $u$ are $j$-th associates $(j=1,2)$.

In the inter-block analysis where only the block totals are used, M. Zelen (11) gives the best linear estimate $\hat{t}_{i}^{\prime}$ of the treatment effect $t_{i}$ as: 


$$
\hat{t}_{i}^{\prime}=\frac{k-c_{2}^{\prime}}{r} Q_{i}^{\prime}+\frac{C_{1}^{\prime}-c_{2}^{\prime}}{r} s_{1}\left(Q_{i}^{\prime}\right),
$$

where:

$$
Q_{i}^{\prime}=T_{i}-Q_{i}-\frac{r G}{N},
$$

and

$$
c_{j}^{\prime}=\frac{c_{j} \Delta-r \lambda_{j}}{\Delta-r H+r^{2}} \quad, \quad(j=1,2) .
$$

In this case,

$$
v\left(\hat{t}_{i}^{\prime}-\hat{t}_{u}^{\prime}\right)=\frac{2\left(k-c_{j}^{\prime}\right)}{r} \sigma^{\prime 2},
$$

where the treatments $i$ and $u$ are $j$-th associates $(j=1,2)$, and where

$$
\sigma^{\prime 2}=\sigma^{2}+k \sigma_{b}^{2}
$$

$\sigma^{2}$ is the error variance in the intra-model, and $\sigma_{b}^{2}$ is the variance of the block effect in the random or inter-model.

\subsection{Variances and Covariances}

From the restriction $\sum_{i=1}^{v} \hat{t}_{i}=0$, the $v\left(\sum_{i=1}^{v} \hat{t}_{i}\right)=0$, or:

$$
v v\left(\hat{t}_{i}\right)+v(v-1) \operatorname{Cov}\left(\hat{t}_{i}, \hat{t}_{j}\right)=0 \text {. }
$$

Since every treatment has $\mathrm{n}_{1}$ first associates and $\mathrm{n}_{2}$ second associates, and $n_{1}+n_{2}=v-1$, then: 


$$
v v\left(\hat{t}_{i}\right)+v n_{1} \operatorname{Cov}\left(\hat{t}_{i}, \hat{t}_{i 1}\right)+v_{2} \operatorname{Cov}\left(\hat{t}_{i}, \hat{t}_{i 2}\right)=0,
$$

or

$$
v\left(\hat{t}_{i}\right)+n_{1} \operatorname{Cov}\left(\hat{t}_{i}, \hat{t}_{i 1}\right)+n_{2} \operatorname{Cov}\left(\hat{t}_{i 1}, \hat{t}_{i 2}\right)=0,
$$

where $\hat{t}_{i j}$ is the $j-t h$ associate of $\hat{t}_{i},(j=1,2)$. From (8.1.8) for $j=1,2$ :

$$
v\left(\hat{t}_{i}\right)+v\left(\hat{t}_{i 1}\right)-2 \operatorname{Cov}\left(\hat{t}_{i}, \hat{t}_{i 1}\right)=\frac{2\left(k-c_{1}\right)}{a} \sigma^{2},
$$

and

$$
v\left(\hat{t}_{i}\right)+v\left(\hat{t}_{i 2}\right)-2 \operatorname{Cov}\left(\hat{t}_{i}, \hat{t}_{i 2}\right)=\frac{2\left(k-c_{2}\right)}{a} \sigma^{2} \text {. }
$$

Let $v\left(\hat{t}_{i}\right)=v_{1}$ for $i=1,2, \ldots, v, \operatorname{Cov}\left(\hat{t}_{i}, \hat{t}_{i 1}\right)=c_{1}$, and $\operatorname{Cov}\left(\hat{t}_{i}, \hat{t}_{i 2}\right)=c_{2}$, then $(8.2 .3)$ may be written as:

$$
\begin{aligned}
\mathrm{v}_{1}+\mathrm{n}_{1} \mathrm{c}_{1}+\mathrm{n}_{2} \mathrm{c}_{2} & =0, \\
2 \mathrm{v}_{1}-2 \mathrm{c}_{1} & =\frac{2\left(k-\mathrm{c}_{1}\right)}{\mathrm{a}} \sigma^{2},
\end{aligned}
$$

and

$$
2 \mathrm{~V}_{1}-\mathrm{CC}_{2}=\frac{2\left(\mathrm{k}-\mathrm{C}_{2}\right)}{\mathrm{a}} \sigma^{2} \text {, }
$$

or

$$
\begin{aligned}
\mathrm{v}_{1}+\mathrm{n}_{1} \mathrm{c}_{1}+\mathrm{n}_{2} \mathrm{c}_{2} & =0 \\
\mathrm{v}_{1}-\mathrm{c}_{1} & =\frac{\mathrm{k}-\mathrm{c}_{1}}{\mathrm{a}} \sigma^{2}, \\
\mathrm{v}_{1}-\mathrm{c}_{2} & =\frac{\mathrm{k}-\mathrm{c}_{2}}{\mathrm{a}} \sigma^{2},
\end{aligned}
$$


By subtracting (8.2.6) from (8.2.5) and (8.2.5) from (8.2.4), one obtains:

$$
c_{2}-c_{1}=\frac{c_{2}-c_{1}}{a} \sigma^{2},
$$

and

$$
\left(n_{1}+1\right) c_{1}+n_{2} c_{2}=\frac{c_{1}-k}{a} \sigma^{2} .
$$

Remembering that $\mathrm{n}_{1}+\mathrm{n}_{2}+1=\mathrm{v},(8.2 .7)$ and (8.2.8) may be written as:

$$
c_{1}=\frac{c_{1}\left(n_{2}+1\right)-n_{2} c_{2}-k}{a v} \sigma^{2},
$$

and

$$
c_{2}=\frac{c_{2}\left(n_{1}+1\right)-n_{1} c_{1}-k}{a v} \sigma^{2}
$$

Substituting (8.2.9) into (8.2.5), one obtains:

$$
\begin{aligned}
\frac{1}{\sigma^{2}} v_{1} & =\frac{k-c_{1}}{a}+\frac{c_{1}\left(n_{2}+1\right)-n_{2} c_{2}-k}{a v} \\
& =\frac{v k-v c_{1}+c_{1}\left(n_{2}+1\right)-n_{2} c_{2}-k}{a v}=\frac{k(v-1)-\left(n_{1}+n_{2}+1\right) c_{1}+\left(n_{2}+1\right) c_{1}-n_{2} c_{2}}{a v},
\end{aligned}
$$

or

$$
\mathrm{v}_{1}=\frac{\mathrm{k}(\mathrm{v}-1)-\mathrm{n}_{1} \mathrm{c}_{1}-\mathrm{n}_{2} \mathrm{c}_{2}}{\mathrm{av}} \sigma^{2}
$$

Since $\sum_{i=1}^{V} \hat{t}_{i}^{\prime}=0$, where $\hat{t}_{i}^{\prime \prime}$ 's are the inter-estimates for 
treatments, one obtains, in exactly the same way:

$$
\begin{aligned}
& v_{1}^{\prime}=\frac{k(v-1)-n_{1} c_{1}^{\prime}-n_{2} c_{2}^{\prime}}{v r} \sigma^{\prime 2}, \\
& c_{1}^{\prime}=\frac{c_{1}^{\prime}\left(n_{2}+1\right)-n_{2} c_{2}^{\prime}-k}{v r} \sigma^{\prime 2}, \\
& c_{2}^{\prime}=\frac{\left(n_{1}+1\right) c_{2}^{\prime}-n_{1} c_{1}^{\prime}-k}{v r} \sigma^{\prime 2},
\end{aligned}
$$

where $v_{1}^{\prime}=v\left(\hat{t}_{i}^{\prime}\right), c_{1}^{\prime}=\operatorname{Cov}\left(\hat{t}_{i}^{\prime}, \hat{t}_{i 1}^{\prime}\right), c_{2}^{\prime}=\operatorname{Cov}\left(\hat{t}_{i}^{\prime}, \hat{t}_{i 2}^{\prime}\right)$, and $\hat{t}_{i j}^{\prime}$ is the $j$-th associate of $\hat{t}_{i}^{\prime},(j=1,2)$. Let

$$
\begin{aligned}
& \mathrm{v}=\mathrm{v}_{1}+\mathrm{v}_{1}^{\prime}, \\
& \mathrm{c}=\mathrm{c}_{1}+\mathrm{c}_{1}^{\prime},
\end{aligned}
$$

and

$$
C^{\prime}=C_{2}+C_{2}^{\prime}
$$

In the combined analysis of Rao, the variance of the difference between two treatments is given in Reference (1) as :

$$
V\left(T_{i}-T_{j}\right)=\frac{2\left(k-d_{j}\right)}{r\left[w^{\prime}+w(k-1)\right]}, \quad(j=1,2) .
$$

Also, here

$$
\begin{gathered}
\sum_{i=1}^{\mathrm{V}} \mathrm{T}_{i}=0, \mathrm{~V}\left[\sum_{i=1}^{\mathrm{V}} \mathrm{T}_{i}\right]=0, \quad \text { or as in }(8.2 .2), \\
\mathrm{V}\left(\mathrm{T}_{i}\right)+\mathrm{n}_{1} \operatorname{Cov}\left(\mathrm{T}_{i}, \mathrm{~T}_{i 1}\right)+\mathrm{n}_{2} \operatorname{Cov}\left(\mathrm{T}_{i}, \mathrm{~T}_{i 2}\right)=0 .
\end{gathered}
$$


(8.2.19), together with the two equations resulted from (8.2.18) for $j=1,2$, give a system of three equations of three unknowns. The work is exactly parallel to that of the intra-estimates, and

$$
V\left(T_{i}\right)=\frac{k(v-1)-n_{1} d_{1}-n_{2} d_{2}}{v r\left[w(k-1)+w^{\prime}\right]},
$$

as it was mentioned in (2.7).

\subsection{Sign of the Quantity $\mathrm{C}-\mathrm{C}^{\prime}$}

By subtracting (8.2.10) from (8.2.9),

$$
\begin{aligned}
c_{1}-c_{2} & =\frac{c_{1}\left(n_{2}+1\right)-n_{2} c_{2}-k-\left(n_{1}+1\right) c_{2}+n_{1} c_{1}+k}{a v} \sigma^{2}, \\
& =\frac{c_{1}\left(n_{1}+n_{2}+1\right)-c_{2}\left(n_{1}+n_{2}+1\right)}{a v}=\frac{c_{1}-c_{2}}{a} \sigma^{2}, \\
& =\frac{k \Delta c_{1}-k \Delta c_{2}}{k \triangle a}, \text { by }(8.1 .4) \text { and }(8.1 .5), \\
& =\frac{a\left(\lambda_{1}-\lambda_{2}\right)}{k \Delta a} \sigma^{2}, \\
& =\frac{\lambda_{1}-\lambda_{2}}{k \Delta} \sigma^{2} .
\end{aligned}
$$

Similarly, by subtracting (8.2.14) from (8.2.13),

$$
\begin{aligned}
c_{1}^{\prime}-c_{2}^{\prime} & =\frac{c_{1}^{\prime}\left(n_{1}+n_{2}+1\right)-c_{2}^{\prime}\left(n_{1}+n_{2}+1\right)}{v r} \sigma^{\prime 2}, \\
& =\frac{c_{1}^{\prime}-c_{2}^{\prime}}{r} \sigma^{\prime 2},
\end{aligned}
$$


where $c_{1}^{\prime}, C_{2}^{\prime}, \sigma^{\prime 2}$ are as defined in (8.1.11) and (8.1.13). Substituting for $\mathrm{c}_{1}^{1}, \mathrm{c}_{2}^{\prime},(8.3 .2)$ may be written,

$$
C_{1}^{\prime}-C_{2}^{\prime}=\frac{\Delta\left(C_{1}-c_{2}\right)-r\left(\lambda_{1}-\lambda_{2}\right)}{r\left(\Delta-r H+r^{2}\right)} \sigma^{\prime 2} \text {. }
$$

Now by adding (8.3.4) and (8.3.1) one obtains:

$$
\begin{aligned}
C-C^{\prime} & =\left(C_{1}+C_{1}^{\prime}\right)-\left(C_{2}+C_{2}^{\prime}\right)=\frac{\lambda_{1}-\lambda_{2}}{k \Delta} \sigma^{2}+\frac{\Delta\left(c_{1}-C_{2}\right)-r\left(\lambda_{1}-\lambda_{2}\right)}{r\left(\Delta-r H+r^{2}\right)} \sigma^{\prime 2}, \\
& =\frac{\lambda_{1}-\lambda_{2}}{k \Delta} \sigma^{2}+\frac{\left[\Delta a\left(\lambda_{1}-\lambda_{2}\right) / k \Delta\right]-r\left(\lambda_{1}-\lambda_{2}\right)}{r\left(\Delta-r H+r^{2}\right)} \sigma^{\prime 2}, \\
& =\frac{\lambda_{1}-\lambda_{2}}{k \Delta} \sigma^{2}+\frac{a\left(\lambda_{1}-\lambda_{2}\right)-k r\left(\lambda_{1}-\lambda_{2}\right)}{r k\left(\Delta-r H+r^{2}\right)} \sigma^{\prime 2},
\end{aligned}
$$

and since $a=r k-r$,

$$
C-C^{\prime}=\frac{\lambda_{1}-\lambda_{2}}{k \Delta} \sigma^{2}-\frac{\lambda_{1}-\lambda_{2}}{k\left(\Delta-r H+r^{2}\right)} \sigma^{\prime 2} \text {. }
$$

Substituting for $\sigma^{\prime 2}$ from (8.1.13),

$$
\begin{aligned}
C-C^{\prime} & =\frac{\lambda_{1}-\lambda_{2}}{k}\left[\frac{1}{\Delta}-\frac{1}{\Delta-r H+r^{2}}\right] \sigma^{2}-\frac{\lambda_{1}-\lambda_{2}}{\Delta-r H+r^{2}} \sigma_{b}^{2}, \\
& =\left(\lambda_{1}-\lambda_{2}\right)\left[\frac{\Delta-r H+r^{2}-\Delta}{k \Delta\left(\Delta-r H+r^{2}\right)} \sigma^{2}-\frac{1}{\Delta-r H+r^{2}} \sigma_{b}^{2}\right], \\
& =\left(\lambda_{1}-\lambda_{2}\right)\left[\frac{-r(H-r)}{k \Delta\left(\Delta-r H+r^{2}\right)} \sigma^{2}-\frac{1}{\Delta-r H+r^{2}} \sigma^{2}\right],
\end{aligned}
$$

Now if

$$
\Delta>0, \Delta-r H+r^{2}>0, \quad H>r,
$$

then $(8.3 .6)$ is of the form: 


$$
C-C^{\prime}=\left(\lambda_{1}-\lambda_{2}\right)[\text { negative quantity }],
$$

i.e.,

$$
\begin{aligned}
& C-C^{\prime}>0 \text { if } \lambda_{1}<\lambda_{2} \text {, } \\
& \mathrm{C}^{-\mathrm{C}^{\prime}\langle 0} \text { if } \lambda_{1}>\lambda_{2} \text {. }
\end{aligned}
$$

It is to be noticed that the above conditions in (8.3.7) are satisfied in every design listed in R.C. Bose "Tables of p.b.i.b. designs with two associate classes", Reference (1).

\subsection{Theorem 3}

As a generalization to Theorem 1 , consider the $t$ independent parameters $\tau_{1}, \tau_{2}, \ldots, \tau_{t}$ and suppose that for each $\tau_{i}$ there exist two independent unbiased estimates $U_{i}$ and $x_{i}$, where $U_{i} \frown N\left(\tau_{i}, v_{i}=\theta_{i} \sigma^{2}\right)$ and $x_{i} \frown N\left(\tau_{i}, v_{i}^{\prime}>v_{i}\right)$. Suppose also that independently of the $\mathrm{x}_{i}$ 's, $U_{i}$ 's there exists an unbiased estimate $s^{2}$ for $\sigma^{2}$ where $s^{2} \frown X^{2} \frac{\sigma^{2}}{f}$, then we can determine a known constant B so that the unbiased combined estimate,

$$
\hat{\tau}_{i}=U_{i}+\frac{\theta_{i} B s^{2}}{\sum_{\substack{j=1 \\ j \neq i}}^{t}\left(x_{j}-U_{j}\right)^{2}}\left(x_{i}-U_{i}\right),
$$

has a variance less than $V\left(U_{i}\right)$ whenever $t>5$. 


\section{Proof:}

$$
E\left(\hat{\tau}_{i}\right)=\tau_{i}+\theta_{i} B \sigma^{2} E \frac{1}{\sum_{j \neq i}^{t}\left(x_{j}-U_{j}\right)^{2}} \cdot E\left(x_{i}-U_{i}\right)=\tau_{i} \quad \text { (8.4.2) }
$$

$$
V\left(\hat{\tau}_{i}\right)=V\left(U_{i}\right)+\theta_{i}^{2} B^{2} E\left(s^{4}\right) \cdot E \frac{z_{i}^{2}}{\left(\sum_{\substack{j=1 \\ j \neq i}}^{t} z_{j}^{2}\right)^{2}}+2 \theta_{i} B E\left(s^{2}\right) \cdot E \frac{z_{i}\left(U_{i}-\tau_{i}\right)}{\sum_{\substack{j=1 \\ j \neq i}}^{t} z_{j}^{2}},
$$

where $z_{j}=x_{j}-U_{j}, \quad z_{i}=x_{i}-U_{i}$.

$$
\begin{aligned}
V\left(\hat{\tau}_{i}\right)=v_{i} & +\frac{\theta_{i}^{2} \sigma^{4} B^{2}(f+2)}{f} E\left(z_{i}^{2}\right) \cdot E \frac{1}{\left(\sum_{j \neq i}^{t} z_{j}^{2}\right)^{2}} \\
& +2 \theta_{i} \sigma^{2} B E \frac{1}{\sum_{j \neq i}^{t} z_{j}^{2}} \cdot E\left[z_{i}\left(U_{i}-\tau_{i}\right)\right],
\end{aligned}
$$

but

$E\left(z_{i}^{2}\right)=E\left[\left(x_{i}-\tau_{i}\right)-\left(U_{i}-\tau_{i}\right)\right]^{2}=E\left(x_{i}-\tau_{i}\right)^{2}+E\left(U_{i}-\tau_{i}\right)^{2}=v_{i}+v_{i}^{\prime}$, substituting into (8.4.3),

$$
\begin{aligned}
V\left(\hat{\tau}_{i}\right)=v_{i} & +\frac{v_{i}^{2} B^{2}(f+2)\left(v_{i}+v_{i}^{\prime}\right)}{f} E \frac{1}{\left(\sum_{j \neq i}^{t} z_{j}^{2}\right)^{2}} \\
& +2 v_{i} B E \frac{1}{\sum_{j \neq i}^{t} z_{j}^{2}} E\left[\left(x_{i}-U_{i}\right)\left(U_{i}-\tau_{i}\right)\right] .
\end{aligned}
$$


Let

$$
\Phi_{i}=v_{i}+v_{i}^{\prime}=v\left(z_{i}\right), \quad i=1,2,3 \ldots t,
$$

then

$$
\begin{aligned}
& v_{i}\left(\hat{\tau}_{i}\right)=v_{i}+\frac{v_{i}^{2} B^{2}(f+2) \Phi_{i}}{f} E \frac{1}{\left(\sum_{j \neq i}^{t} z_{j}^{2}\right)^{2}}+2 v_{i} B E\left(\frac{1}{t}\right)\left(-v_{i}\right) \\
& =v_{i}+\frac{v_{i}^{2} B(f+2) \Phi_{i}}{f} \frac{1}{z_{j \neq i}^{2}} \\
& \left(\sum_{j \neq i}^{t} z_{j}^{2}\right)^{2} \\
& \sum_{j \neq i} z_{j}^{2}
\end{aligned}
$$

It should be noticed that $z_{j} \cap N\left(0, \Phi_{j}\right)$. Let $z_{j}=\sqrt{\Phi_{j}} \cdot Y_{j}$, then, $Y_{j} \cap N(0,1), \sum_{j \neq i}^{t} Y_{j}^{2} \cap X_{(t-1)}^{2}, \quad$ and,

$$
\sum_{j \neq i}^{t} z_{j}^{2}=\sum_{j \neq i}^{t} \Phi_{j} Y_{j}^{2} .
$$

Now one can write:

$$
\begin{gathered}
E \frac{1}{\left(\sum_{j \neq i}^{t} z_{j}^{2}\right)^{2}} \leq \mathrm{E} \frac{1}{\Phi_{\min .}^{2}\left(\sum_{j \neq i}^{t} Y_{j}^{2}\right)^{2}}=\frac{1}{\Phi_{\min }^{2}} E\left(\frac{1}{\chi_{(t-1)}^{2}}\right)^{2} \\
=\frac{1}{\Phi_{\min }^{2}(t-3)(t-5)},
\end{gathered}
$$


and

$E \frac{1}{\sum_{j \neq i}^{t} z_{j}^{2}} \geq \pm \frac{1}{\Phi_{\max } \sum_{j \neq i}^{t} Y_{j}^{2}}=\frac{1}{\Phi_{\max .}} \cdot E \frac{1}{X_{(t-1)}^{2}}=\frac{1}{\Phi_{\max }(t-3)}$

Using (8.4.8) and (8.4.9), we can write $(8.4 .6)$ as:

$$
\mathrm{V}\left(\hat{\tau}_{i}\right)<v_{i}+\frac{\mathrm{V}_{i}^{2} B^{2}(f+2) \Phi_{i}}{f \Phi_{\min }^{2}(t-3)(t-5)}-\frac{2 V_{i}^{2} B}{\Phi_{\max .}(t-3)} \cdot(8.4 .10)
$$

In (8.4.10) we want the quadratic in $B$, in the last two terms to be negative, i.e.,

$$
\frac{V_{i}^{2} B}{t-3}\left[\frac{B(f+2) \Phi_{i}}{f \Phi_{\text {min. }}^{2}(t-5)}-\frac{2}{\Phi_{\max .}}\right]<0,
$$

or

$$
\begin{aligned}
& 0<B<\frac{2 f \Phi_{\min }^{2}(t-5)}{(f+2) \Phi_{i} \cdot \Phi_{\max } .}, \quad t>5 . \\
& B_{\text {opt. }}=\frac{f \Phi_{\min }^{2}(t-5)}{(f+2) \Phi_{i} \cdot \Phi_{\max } .},
\end{aligned}
$$

where $B_{\text {opt. }}$ is the value of $B$ which minimizes the above quadratic.

$$
\begin{aligned}
\text { Since } \Phi_{i} \leq \Phi_{\max .}, \text { one can write: } \\
B=\frac{f(t-5)}{f+2} \cdot\left(\frac{\Phi_{\min .}}{\Phi_{\max .}}\right)^{2},
\end{aligned}
$$

as an admissible $B$, i.e., within the above range of $B$ which makes $V\left(\hat{\tau}_{i}\right)<V\left(U_{i}\right)$. 
In the incomplete block designs where both inter- and intra-block estimates are available and independent, the variance is of the form $\alpha_{1} \sigma^{2}+\alpha_{2} \sigma_{b}^{2}$. Let $\frac{\sigma_{b}^{2}}{\sigma^{2}}=R$, and knowing that $\sigma_{b}^{2}$ should never be less than $\sigma^{2}$ in any reasonable incomplete block design,

$$
\frac{\Phi_{\min }}{\Phi_{\max }}=\frac{\alpha_{1} \sigma^{2}+\alpha_{2} \sigma_{b}^{2}}{\gamma_{1} \sigma^{2}+\gamma_{2} \sigma_{b}^{2}}=\frac{\alpha_{1}+\alpha_{2} R}{\gamma_{1}+\gamma_{2} R}=F(R),
$$

where $\alpha_{1}, \alpha_{2}, \gamma_{1}, \gamma_{2}$ are known in terms of the parameters of the design.

$F(R)$ is a hyperbolic function; it is monotonically decreasing if $\alpha_{2} \gamma_{1}<\alpha_{1} \gamma_{2}$, and monotonically increasing if $\alpha_{2} \gamma_{1}>\alpha_{1} \gamma_{2}$

$$
\begin{aligned}
& \text { Now if } \alpha_{2} \gamma_{1}<\alpha_{1} \gamma_{2}, \text { then } \\
& F(\infty)<F(R)<F(1),
\end{aligned}
$$

and from (8.4.13),

$$
\frac{\Phi_{\min .}}{\Phi_{\max .}}=F(R)>F(\infty)=\frac{\alpha_{2}}{\gamma_{2}} .
$$

In this case,

$$
B=\frac{f(t-5)}{f+2} \frac{\alpha_{2}}{\gamma_{2}} .
$$

If $\alpha_{2} \gamma_{1}>\gamma_{2} \alpha_{1}$, then,

$$
F(1)<F(R)<F(\infty),
$$


and from (8.4.13),

$$
\frac{\Phi_{\min .}}{\Phi_{\max .}}=F(R)>F(1)=\frac{\alpha_{1}+\alpha_{2}}{\gamma_{1}+\gamma_{2}} \text {. }
$$

In this case

$$
B=\frac{f(t-5)}{f+2} \frac{\alpha_{1}+\alpha_{2}}{\gamma_{1}+\gamma_{2}} .
$$

In both cases B can be computed from the parameters of our design.

\subsection{Special Case}

When there are only two different variances, let $t_{1}$ of the $z$ 's have variance $\Phi_{1}$ and let the remaining $t-t_{1}$ have variance $\Phi_{2}$, then $(8.4 .7)$ becomes:

$$
\begin{gathered}
\sum_{j \neq i}^{t} z_{j}^{2}=\Phi_{1} x^{2}\left(t_{1}-1\right)+\Phi_{2} x^{2}\left(t-t_{1}\right)=\Phi_{1} x^{2}\left(v_{1}\right)+\Phi_{2} X^{2}\left(v_{2}\right), \\
\text { when } v\left(z_{i}\right)=\Phi_{1}, \\
\sum_{j \neq i}^{t} z_{j}^{2}=\Phi_{1} x^{2}\left(t_{1}\right)+\Phi_{2} x^{2}\left(t-t_{1}-1\right)=\Phi_{1} x^{2}\left(v_{1}\right)+\Phi_{2} X^{2}\left(v_{2}\right), \\
\text { when } v\left(z_{i}\right)=\Phi_{2} .
\end{gathered}
$$

It should be noticed that $v_{1}$ and $v_{2}$ assume different values in the two cases, but $v_{1}+v_{2}=t-1$ always. 
In both cases let us assume that

$$
\sum_{\substack{j=1 \\ j \neq i}}^{h} z_{j}^{2}=\Phi_{1} \chi_{v_{1}}^{2}+\Phi_{2} \chi_{v_{2}}^{2}=x+y,
$$

where

$$
f(x)=\frac{1}{\Gamma\left(\frac{1}{2} \nu_{1}\right)\left(2 \Phi_{1}\right)^{\frac{1}{2} \nu_{1}}} x^{\frac{1}{2} \nu_{1}-1} e^{-x / 2 \Phi_{1}} d x, \quad x>0, \Phi_{1}>0,
$$

and

$$
f(Y)=\frac{1}{\Gamma\left(\frac{1}{2} \nu_{1}\right)\left(2 \Phi_{2}\right)^{\frac{1}{2} \nu_{2}}} y^{\frac{1}{2} \nu} 2^{-1} e^{-y / 2 \Phi_{2}} d y, \quad y>0, \quad \Phi_{2}>0 .
$$

Applying lemma 1, we have

$$
\beta_{1}=2 \Phi_{1}, \alpha_{1}=\frac{1}{2} \nu_{1}-1, \beta_{2}=2 \Phi_{2}, \alpha_{2}=\frac{1}{2} \nu_{2}-1,
$$

thus substituting into (4.6) we get:

$$
\begin{aligned}
& E\left(\frac{1}{X+Y}\right)^{r}=\frac{\Gamma\left(\frac{1}{2} \bar{\nu}_{1}+\nu_{2}-r\right)}{\Gamma\left(\frac{1}{2} \nu_{1}\right) \Gamma\left(\frac{1}{2} \nu_{2}\right)} \int_{0}^{1} \frac{u^{\frac{1}{2} \nu_{1}-1}(1-u)^{\frac{1}{2} \nu_{2}-1}}{\left[2 \Phi_{1} u+2 \Phi_{2}(1-u)\right]^{r}} d u, \\
& =\frac{\Gamma\left(\frac{1}{2} \nu_{1}+v_{2}-r\right)}{2^{r} \Gamma\left(\frac{1}{2} v_{1}\right) \Gamma\left(\frac{1}{2} v_{2}\right) \Phi_{1}^{r}} \int_{0}^{1} \frac{u^{\frac{1}{2} v_{1}-1}(1-u)^{\frac{1}{2} v_{2}} 2^{-1}}{\left[u+\frac{\Phi_{2}}{\Phi_{1}}(1-u)\right]^{r}} d u
\end{aligned}
$$

Suppose that one is able to find a lower bound $L$ and an upper bound $P$ for the ratio $\frac{\Phi_{2}}{\Phi_{1}}$ which are relatively not 
far from each other, then one can write:

$$
\begin{aligned}
& \mathrm{E}\left(\frac{1}{\mathrm{X}+\mathrm{Y}}\right)^{2} \leq \frac{\Gamma\left(\frac{1}{2} v_{1}+v_{2}-2\right)}{4 \Gamma\left(\frac{1}{2} v_{1}\right) \Gamma\left(\frac{1}{2} v_{2}\right) \Phi_{1}^{2}} \int_{0}^{1} \frac{\mathrm{u}^{\frac{1}{2} v_{1}-1}(1-u)^{\frac{1}{2} v_{2}} 2^{-1}}{[u+L(1-u)]^{2}} d u,
\end{aligned}
$$

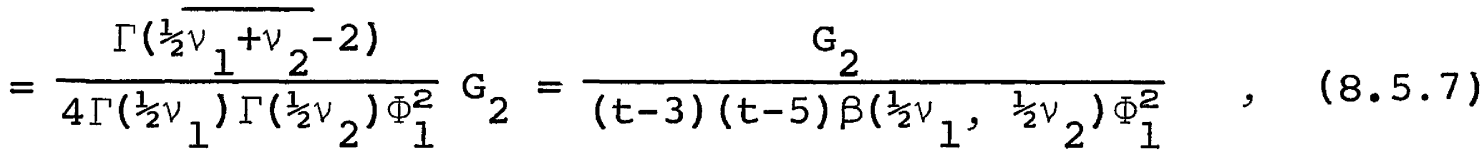

and

$E \frac{1}{X+Y} \geq \frac{\Gamma\left(\frac{1}{2} \bar{\nu}_{1}+\nu_{2}-1\right)}{2 \Gamma\left(\frac{1}{2} \nu_{1}\right) \Gamma\left(\frac{1}{2} \nu_{2}\right) \Phi_{1}} \int_{0}^{1} \frac{u^{\frac{1}{2} \nu} 1^{-1}(1-u)^{\frac{1}{2} \nu} 2^{-1}}{u+P(1-u)} d u$,

$$
=\frac{\Gamma\left(\frac{1}{2} \bar{\nu}_{1}+v_{2}-1\right)}{2 \Gamma\left(\frac{1}{2} \nu_{1}\right) \Gamma\left(\frac{1}{2} \nu_{2}\right) \Phi_{1}} G_{1}=\frac{\mathrm{G}_{1}}{(t-3) \beta\left(\frac{1}{2} \nu_{1}, \frac{1}{2} \nu_{2}\right) \Phi_{1}} .
$$

Accounting for (8.5.3), and substituting (8.5.7) and (8.5.8) into $(8.4 .6)$ :

$$
\begin{aligned}
& v\left(\hat{\tau}_{i}\right)<v_{i}+\frac{v_{i}^{2} B^{2}(f+2) \Phi_{i} G_{2}}{f(t-3)(t-5) \beta\left(\frac{1}{2} v_{1}, \frac{1}{2} \nu_{2}\right) \Phi_{1}^{2}}-\frac{2 v_{i}^{2} B G_{1}}{(t-3) \beta\left(\frac{1}{2} \nu_{1}, \frac{1}{2} \nu_{2}\right) \Phi_{1}}, \\
& =v_{i}+\frac{v_{i}^{2} B}{(t-3) \beta\left(\frac{1}{2} \nu_{1}, \frac{1}{2} \nu_{2}\right) \Phi_{1}}\left[\frac{B(f+2) \Phi_{i} G_{2}}{f(t-5) \Phi_{1}}-2 G_{1}\right] .
\end{aligned}
$$

Assuming $t>5$, the second term is negative when

$$
0<B<\frac{2 f(t-5) G_{1} \Phi_{1}}{(f+2) \Phi_{i} G_{2}} .
$$

The value $B_{\text {opt. }}$ which makes it the most negative is: 


$$
B_{\text {opt. }}=\frac{f \Phi_{1} G_{1}(t-5)}{\Phi_{i} G_{2}(f+2)}
$$

Since $\Phi_{i}$ is either $\Phi_{1}$ or $\Phi_{2}$ in this special case, we have

$$
B_{\text {opt. }}=\frac{f G_{1}(t-5)}{(f+2) G_{2}} \text {, when } \Phi_{i}=\Phi_{1} \text {. }
$$

However, (8.5.11) depends on the variances $\Phi_{1}, \Phi_{2}$ when $\Phi_{i}=\Phi_{2}$; to avoid this, we go back to (8.5.6) and take $\Phi_{2}$ outside the integral instead of $\Phi_{1}$ to get:

$E\left(\frac{1}{X+Y}\right)^{r}=\frac{\Gamma\left(\frac{1}{2} \bar{\nu}_{1}^{+\nu_{2}}-r\right)}{2^{r} \Gamma\left(\frac{1}{2} \nu_{1}\right) \Gamma\left(\frac{1}{2} \nu_{2}\right) \Phi_{2}^{r}} \int_{0}^{1} \frac{u^{\frac{1}{2} \nu_{1}-1}(1-u)^{\frac{1}{2} \nu_{2}-1}}{\left[\frac{1}{\Phi_{2}} u+(1-u)\right]^{r}} d u \cdot(8.5 .13)$ The lower and upper bounds for $\frac{\Phi_{2}}{\Phi_{1}}$ are $\frac{1}{\mathrm{P}}$ and $\frac{1}{\mathrm{~L}}$ respectively, hence using (8.5.13):

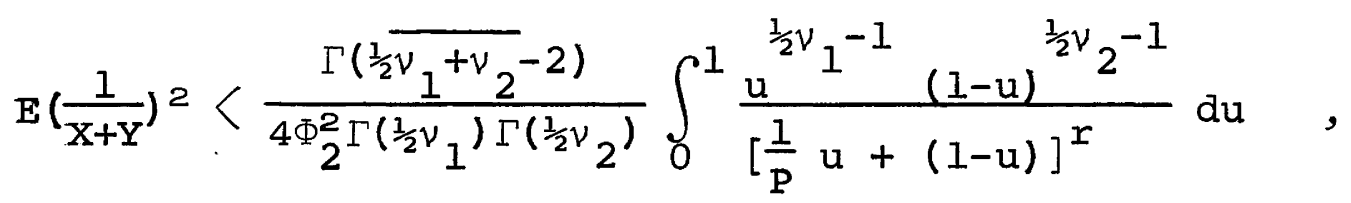

$=\frac{\Gamma\left(\frac{1}{2} \bar{\nu}_{1}+v_{2}-2\right) G_{2}^{\prime}}{4 \Phi_{2}^{2} \Gamma\left(\frac{1}{2} \nu_{1}\right) \Gamma\left(\frac{1}{2} \nu_{2}\right)}=\frac{G_{2}^{\prime}}{(t-3)(t-5) \beta\left(\frac{1}{2} \nu_{1}, \frac{1}{2} \nu_{2}\right) \Phi_{2}^{2}}$.

$\mathrm{E}\left(\frac{1}{\mathrm{X}+\mathrm{Y}}\right)>\frac{\Gamma\left(\frac{1}{2} \overline{\nu_{1}+\nu_{2}}-1\right)}{2 \Phi_{2} \Gamma\left(\frac{1}{2} \nu_{1}\right) \Gamma\left(\frac{1}{2} \nu_{2}\right)} \int_{0}^{1} \frac{\mathrm{u}^{\frac{1}{2} \nu_{1}-1}(1-u)^{\frac{1}{2} \nu} 2^{-1}}{\frac{1}{L} u+(1-u)} d u$,

$=\frac{\Gamma\left(\frac{1}{2} \nu_{1}+v_{2}-1\right) G_{1}^{\prime}}{2 \Gamma\left(\frac{1}{2} \nu_{1}\right) \Gamma\left(\frac{1}{2} \nu_{2}\right) \Phi_{2}}=\frac{G_{1}^{\prime}}{(t-3) \beta\left(\frac{1}{2} \nu_{1}, \frac{1}{2} \nu_{2}\right) \Phi_{2}}$. 
Substituting in (8.5.7) for the case, where $\Phi_{i}=\Phi_{2}$ : $v\left(\hat{\tau}_{i}\right)<v_{i}+\frac{v_{i}^{2} B^{2}(f+2) \Phi_{2} G_{2}^{\prime}}{f(t-3)(t-5) \beta\left(\frac{1}{2} \nu_{1}, \frac{1}{2} \nu_{2}\right) \Phi_{2}^{2}}-\frac{2 v_{i}^{2} B G_{1}^{\prime}}{(t-3) \beta\left(\frac{1}{2} \nu_{1}, \frac{1}{2} \nu_{2}\right) \Phi_{2}}$, $=v_{i}+\frac{V_{i}^{2} B}{(t-3) B\left(\frac{1}{2} V_{1}, \frac{1}{2} V_{2}\right) \Phi_{2}}\left[\frac{B(f+2) G_{2}^{\prime}}{f(t-5)}-2 G_{1}^{1}\right]$.

Assuming $t>5$, the second term is negative when

$$
0<B<\frac{2 f(t-5) G_{1}^{\prime}}{(f+2) G_{2}^{\prime}}
$$

and

$$
B_{\text {opt. }}=\frac{f(t-5) G_{1}^{\prime}}{(f+2) G_{2}^{\prime}}
$$

This is independent of the $\Phi^{\prime} \mathrm{s}$, and (8.5.18) will be used when $\Phi_{i}=\Phi_{2}$. Substituting $(8.5 .12)$ into (8.5.9), we get for the case $\Phi_{i}=\Phi_{1}$ :

$$
\mathrm{V}\left(\hat{\tau}_{i}\right)<v_{i}+\frac{v_{i}^{2} f G_{1}(t-5)}{(f+2) G_{2}(t-3) \beta\left(\frac{1}{2} v_{1}, \frac{1}{2} v_{2}\right) \Phi_{1}}\left[G_{1}-2 G_{1}\right],
$$

or

$$
\begin{gathered}
\mathrm{v}(\hat{\tau})<v_{i}-\frac{f(t-5) G_{1}^{2}}{(f+2) G_{2}(t-3) \beta\left(\frac{1}{2} v 1, \frac{1}{2} v_{2}\right)} \cdot \frac{v_{i}^{2}}{\Phi_{1}} \cdot(8.5 .19) \\
\text { But } \frac{v_{i}^{2}}{\Phi_{1}}=\frac{v_{i}^{2}}{v_{i}+V_{i}^{1}} \text { is the utmost possible recovery. Thus the }
\end{gathered}
$$
recovery ratio is at least: 


$$
D=\frac{f(t-5) G_{1}^{2}}{(f+2) G_{2}(t-3) \beta\left(\frac{1}{2} \nu_{1}, \frac{1}{2} \nu_{2}\right)}
$$

Similarly substituting (8.5.18) into (8.5.16), we get for the case $\Phi_{i}=\Phi_{2}$ :

$$
\begin{aligned}
& V\left(\hat{\tau}_{i}\right)<v_{i}+\frac{V_{i}^{2} f(t-5) G_{1}^{\prime}}{(t-3) \beta\left(\frac{1}{2} \nu_{1}, \frac{1}{2} \nu_{2}\right) \Phi_{2}(f+2) G_{2}^{\prime}}\left[G_{1}^{\prime}-2 G_{1}^{\prime}\right], \\
& =V_{i}-\frac{f(t-5) G_{1}^{\prime 2}}{(f+2) G_{2}^{\prime}(t-3) \beta\left(\frac{1}{2} V_{1}, \frac{1}{2} V_{2}\right)} \cdot \frac{V_{i}^{2}}{\Phi_{2}} \text {. }
\end{aligned}
$$
recovery ratio is at least:

$$
D=\frac{f(t-5) G_{1}^{\prime 2}}{(f+2) G_{2}^{\prime}(t-3) \beta\left(\frac{1}{2} \nu_{1}, \frac{1}{2} \nu_{2}\right)} .
$$

8.6 Recovery of Inter-block Information in Group Divisible Designs

In this case $\mathrm{v}=\mathrm{mn}$, and the treatments can be divided into $\mathrm{m}$ groups of $\mathrm{n}$ treatments each, such that any two treatments of the same group are first associates, while two treatments of different groups are second associates. The association scheme can be displayed by placing the treatments in a rectangular of $\mathrm{m}$ rows and $\mathrm{n}$ columns, where each 
row of $\mathrm{n}$ treatments constitutes a group. Clearly:

$$
\mathrm{n}_{1}=\mathrm{n}-1, \quad \mathrm{n}_{2}=\mathrm{n}(\mathrm{m}-1) \quad \text {. }
$$

Further:

$$
(n-1) \lambda_{1}+n(m-1) \lambda_{2}=r(k-1),
$$

or

$$
r k-\lambda_{2} v=r-\lambda_{1}+n\left(\lambda_{1}-\lambda_{2}\right)
$$

Also:

$$
P_{1}=\left(\begin{array}{cc}
n-2 & 0 \\
0 & n(m-1)
\end{array}\right), \quad P_{2}=\left(\begin{array}{cc}
0 & n-1 \\
n-1 & n(m-2)
\end{array}\right) .
$$

Bose and Connor have shown that the following inequalities hold in group divisible (G.D.) designs:

$$
r \geq \lambda_{1}, \quad r k-\lambda_{2} v \geq 0
$$

They have divided the G.D. into three subclasses:

$$
\begin{aligned}
& \text { (i) Singular (S) if } r=\lambda_{1} \text {, } \\
& \text { (ii) Semi-regular (SR) if } r>\lambda_{1} \text { and } r k-\lambda_{2} v=0 \text {, } \\
& \text { (iii) Regular (R) if } r>\lambda_{1} \text { and } r k-\lambda_{2} v>0 .
\end{aligned}
$$

\subsubsection{Recovery in Regular Group Divisibles}

In regular G.D. designs we define the $\mathrm{mn} x \mathrm{mn}$ matrix $M_{1}$ as follows: 


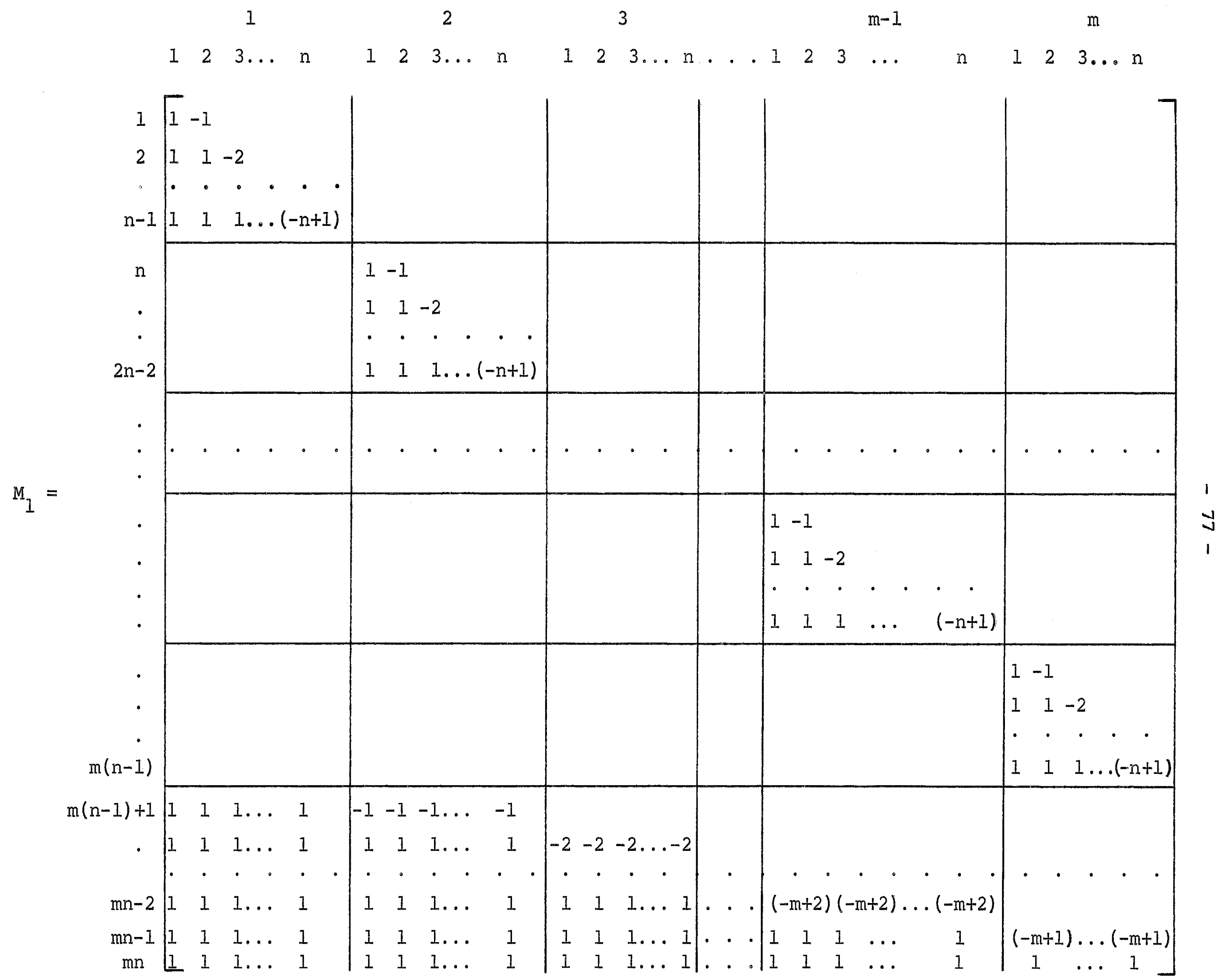


Define the following contrasts between the $\mathrm{mn}$ intra-block estimates of the treatments $\hat{t}_{1}, \hat{t}_{2}, \ldots, \hat{t}_{v}$ :

$$
\underline{U}=\left(\begin{array}{l}
U_{1} \\
U_{2} \\
\vdots \\
U_{n m-1} \\
0
\end{array}\right)=M\left(\begin{array}{l}
\hat{t}_{1} \\
\hat{t}_{2} \\
\vdots \\
\hat{t}_{n m}
\end{array}\right)=M \underline{\underline{t}} \text {, }
$$

where $M$ is the same as $M_{1}$ after normalizing its rows. Define also the similar contrasts between the inter-block estimates of the treatments, namely $\hat{t}_{1}^{\prime}, \hat{t}_{2}^{\prime}, \ldots, \hat{t}_{V}^{\prime}$ :

$$
\underline{x}=\left(\begin{array}{l}
x_{1} \\
x_{2} \\
\vdots \\
x_{n m-1} \\
0
\end{array}\right)=M \underline{\underline{t}}^{\prime}=M\left(\begin{array}{c}
\hat{t}_{1}^{\prime} \\
\vdots \\
\hat{t}_{n m}^{\prime}
\end{array}\right) \cdot(8.6 .7)
$$

The problem now is to combine $\underline{U}$ and $\underline{x}$ to get new estimates $\hat{\underline{\tau}}=\left(\begin{array}{l}\hat{\tau}_{1} \\ \vdots \\ \hat{\tau}_{\mathrm{nm}}\end{array}\right)$. It should be noted that $\mathrm{U}_{\mathrm{nm}}=0, \mathrm{x}_{\mathrm{nm}}=0$, and the corresponding combined estimate is assumed to be zero, i.e., $\hat{\tau}_{\mathrm{nm}}=0$. 
For the variance-covariance matrix of both $\underline{U}$ and $\underline{x}$, it is noticed first that $U_{i}$ is uncorrelated with $U_{j}$ for $i \neq j$; also $x_{i}$ is uncorrelated with $x_{j}$ for $i \neq j$, and the $U_{i}$ 's and $\mathrm{x}_{j}$ 's are independent of each other for every $i, j$, by virtue of the well known fact that the inter-block estimates are independent from the intra-block estimates.

It should also be noted that in the vector $\hat{t}$ or $\hat{t}^{\prime}$ we are grouping the treatments according to group divisible association plan, i.e., the first $\mathrm{n}$ are the first group (or row) in the plan, the next $\mathrm{n}$ treatments are the second row in the association plan, etc.; moreover, it should be noted that the contrasts included in the matrix $M$ are of two kinds, within group contrasts and among group contrasts. It has been shown in (7.2.4) that all within contrasts have exactly the same variance, namely $\mathrm{v}_{1}-\mathrm{C}_{1}$, where $\mathrm{v}_{1}$ is the variance of the intra-treatment estimate and $C_{1}$ is the covariance between two first associate intra-estimates. For the variance of the contrasts among groups, let us take a general one, $\mathrm{U}_{\mathrm{mn}-2}$, say. Then 


$$
\begin{aligned}
& \mathrm{V}\left(\mathrm{U}_{\mathrm{mn}-2}\right)=\frac{1}{\mathrm{n}(\mathrm{m}-2)(\mathrm{m}-1)}\left[\mathrm{n}(\mathrm{m}-2) \mathrm{v}_{1}+\mathrm{n}(\mathrm{m}-2)^{2} \mathrm{v}_{1}+(\mathrm{m}-2) \mathrm{n}(\mathrm{n}-1) \mathrm{c}_{1}\right. \\
& \left.+(m-2)^{2}(n-1) n c_{1}+(m-2)(m-3) n^{2} c_{2}-2(m-2) n^{2}(m-2) c_{2}\right], \\
& =\frac{1}{n(m-2)(m-1)}\left\{n(m-2)(m-1) v_{1}+(n-1) c_{1}\left[n(m-2)+n(m-2)^{2}\right]\right. \\
& \left.+n^{2}(m-2) c_{2}[(m-3)-2(m-2)]\right\}=v_{1}+(n-1) c_{1}-n c_{2}, \\
& =\left(\mathrm{V}_{1}-\mathrm{C}_{1}\right)+\mathrm{n}\left(\mathrm{C}_{1}-\mathrm{C}_{2}\right),
\end{aligned}
$$

where $\mathrm{C}_{2}$ is the covariance between two second associate intra-estimates. Now $\mathrm{V}\left(\mathrm{U}_{\mathrm{mn}-2}\right)$ is independent of $\mathrm{m}$, which means that all among contrasts have the same variance.

Similarly, one can deduce the variances of the $\mathrm{x}^{\prime} \mathrm{s}$, it is $V_{1}^{\prime}-C_{1}^{\prime}$ for within contrasts and $V_{1}^{\prime}-C_{1}^{\prime}+n\left(C_{1}^{\prime}-C_{2}^{\prime}\right)$ for among contrasts, where $V_{1}^{\prime}$ is the variance of the inter-treatment estimate, and $C_{1}^{\prime}, C_{2}^{\prime}$ are the covariances between two, first or second associate inter-estimates respectively.

Consider now the vector:

$$
\underline{\mathrm{z}}=\left(\begin{array}{l}
\mathrm{z}_{1} \\
\mathrm{z}_{2} \\
\vdots \\
\mathrm{z}_{\mathrm{mn}-1}
\end{array}\right)=\underline{\mathrm{x}}-\underline{\mathrm{U}},
$$

$z_{i}$ and $z_{j}$ are independent for $i \neq j$ 
For within group z's, one has

$$
\begin{aligned}
v\left(z_{i}\right) & =v\left(x_{i}\right)+v\left(U_{i}\right)=\left(v_{1}-C_{1}\right)+\left(v_{1}^{\prime}-C_{1}^{\prime}\right), \\
& =\left(v_{1}+v_{1}^{\prime}\right)-\left(C_{1}+C_{1}^{\prime}\right)=v-c .
\end{aligned}
$$

For among group z's, one has

$$
\begin{aligned}
V\left(z_{i}\right) & =v_{1}-C_{1}+n\left(c_{1}-C_{2}\right)+v_{1}^{\prime}-C_{1}^{\prime}+n\left(C_{1}^{\prime}-c_{2}^{\prime}\right), \\
& =v_{1}+v_{1}^{\prime}-\left(c_{1}+C_{1}^{\prime}\right)+n\left[\left(C_{1}+C_{1}^{\prime}\right)-\left(c_{2}+C_{2}^{\prime}\right)\right], \\
& =v-C+n\left(C-C^{\prime}\right),
\end{aligned}
$$

where $\mathrm{V}, \mathrm{C}, \mathrm{C}^{\prime}$ are defined in (8.2.15) through (8.2.17). From $(8.3 .8)$, it is seen that

$$
\mathrm{V}-\mathrm{C}>(\mathrm{V}-\mathrm{C})+\mathrm{n}\left(\mathrm{C}-\mathrm{C}^{\prime}\right) \text {, if } \lambda_{1}>\lambda_{2} \text {, }
$$

and

$$
\mathrm{v}-\mathrm{C}<(\mathrm{v}-\mathrm{C})+\mathrm{n}\left(\mathrm{C}-\mathrm{C}^{\prime}\right) \text {, if } \lambda_{1}<\lambda_{2} \text {. }
$$

This will give rise to two divisions of the problem:

(i) Regular G.D. with $\lambda_{1}>\lambda_{2}$,

(ii) Regular G.D. with $\lambda_{1}<\lambda_{2}$.

\subsubsection{Study of the Ratio $(\mathrm{V}-\mathrm{C}) /\left[\mathrm{V}-\mathrm{C}+\mathrm{n}\left(\mathrm{C}-\mathrm{C}^{\prime}\right)\right]$}

Subtracting (8.2.9) from (8.2.11),

$$
\begin{aligned}
v_{1}-c_{1} & =\left[\frac{k(v-1)-n_{1} c_{1}-n_{2} c_{2}}{a v}-\frac{c_{1}\left(n_{2}+1\right)-n_{2} c_{2}-k}{a v}\right] \sigma^{2}, \\
& =\frac{k v-c_{1}\left(n_{1}+n_{2}+1\right)}{a v} \sigma^{2}=\frac{k-c_{1}}{a} \sigma^{2} .
\end{aligned}
$$


Subtracting (8.2.13) from (8.2.12),

$$
\begin{aligned}
v_{1}^{\prime}-c_{1}^{\prime} & \left.=\frac{k(v-1)-n_{1} c_{1}^{\prime}-n_{2} c_{2}^{\prime}}{v r}-\frac{c_{1}^{\prime}\left(n_{2}+1\right)-n_{2} c_{2}^{\prime}-k}{v r}\right) \sigma^{\prime 2}, \\
& =\frac{k v-c_{1}^{\prime}\left(n_{1}+n_{2}+1\right)}{v r} \sigma^{\prime 2}=\frac{k-c_{1}^{\prime}}{r} \sigma^{\prime 2} \cdot(8.6 .15)
\end{aligned}
$$

Adding (8.6.14) and (8.6.15),

$$
\mathrm{V}-\mathrm{C}=\mathrm{v}_{1}-\mathrm{C}_{1}+\mathrm{V}_{1}^{\prime}-\mathrm{C}_{1}^{\prime}=\frac{\mathrm{k}-\mathrm{C}_{1}}{\mathrm{a}} \sigma^{2}+\frac{\mathrm{k}-\mathrm{C}_{1}^{\prime}}{\mathrm{r}} \sigma^{\prime 2} \text {. }
$$

By (8.3.5) one can write:

$V-C+n\left(C-C^{\prime}\right)=\left[\frac{k-c_{1}}{a}+\frac{n\left(\lambda_{1}-\lambda_{2}\right)}{k \Delta}\right] \sigma^{2}+\left[\frac{k-c_{1}^{\prime}}{r}-\frac{n\left(\lambda_{1}-\lambda_{2}\right)}{k\left(\Delta-r H+r^{2}\right)}\right] \sigma^{\prime 2}$.

From (8.6.4) one obtains $f=p_{12}^{1}=0, g=p_{12}^{2}=n-1$, and substituting in (8.1.2),

$$
\begin{aligned}
\mathrm{k}^{2} \Delta & =\left(a+\lambda_{1}\right)\left(a+\lambda_{2}\right)+\left(\lambda_{1}-\lambda_{2}\right)\left(-a-\lambda_{1}\right)(n-1), \\
& =\left(a+\lambda_{1}\right)\left[a+\lambda_{2}-(n-1)\left(\lambda_{1}-\lambda_{2}\right)\right], \\
& =\left(a+\lambda_{1}\right)\left[a+\lambda_{1}-n\left(\lambda_{1}-\lambda_{2}\right)\right],
\end{aligned}
$$

and using (8.6.3), one can write

$$
\mathrm{k}^{2} \Delta=\left(\mathrm{a}+\lambda_{1}\right) \lambda_{2} \mathrm{v}
$$

Substituting $f$ and $g$ in (8.1.3),

$$
\begin{aligned}
\mathrm{kH} & =\left(2 a+\lambda_{1}+\lambda_{2}\right)-n\left(\lambda_{1}-\lambda_{2}\right)+\lambda_{1}-\lambda_{2}, \\
& =a+\lambda_{1}+\left[a+\lambda_{1}-n\left(\lambda_{1}-\lambda_{2}\right)\right],
\end{aligned}
$$


and using (8.6.3), one obtains

$$
\mathrm{kH}=\mathrm{a}+\lambda_{1}+\lambda_{2} \mathrm{v} .
$$

Substituting $f$ and $g$ in (8.1.4),

$$
\begin{aligned}
\mathrm{k} \Delta c_{1} & =\lambda_{1}\left(a+\lambda_{2}\right)-(n-1) \lambda_{1}\left(\lambda_{1}-\lambda_{2}\right), \\
& =\lambda_{1}\left[a+\lambda_{2}-n\left(\lambda_{1}-\lambda_{2}\right)+\lambda_{1}-\lambda_{2}\right], \\
& =\lambda_{1}\left[a+\lambda_{1}-n\left(\lambda_{1}-\lambda_{2}\right)\right],
\end{aligned}
$$

by $(8 \cdot 6 \cdot 3)$ :

$$
k \Delta c_{1}=\lambda_{1} \lambda_{2} \mathrm{v}
$$

subtracting (8.1.5) from (8.1.4),

$$
k \Delta\left(c_{1}-c_{2}\right)=\lambda_{1}\left(a+\lambda_{2}\right)-\lambda_{2}\left(a+\lambda_{1}\right)=a\left(\lambda_{1}-\lambda_{2}\right),
$$

or

$$
c_{1}-c_{2}=\frac{a k\left(\lambda_{1}-\lambda_{2}\right)}{k^{2} \Delta}
$$

and using (8.6.18), one obtains:

$$
c_{1}-c_{2}=\frac{k a\left(\lambda_{1}-\lambda_{2}\right)}{\lambda_{2} v\left(a+\lambda_{1}\right)} \text {. }
$$

Now using (8.6.18) and (8.6.20),

$$
\begin{aligned}
\frac{k-c_{1}}{a} & =\frac{k^{2} \Delta-k \Delta c_{1}}{a k \Delta}=\frac{a \lambda_{2} v}{a k \Delta}=\frac{\lambda_{2} v k}{k^{2} \Delta}=\frac{\lambda_{2} v k}{\lambda_{2} v\left(a+\lambda_{1}\right)}, \\
& =\frac{k}{a+\lambda_{1}} .
\end{aligned}
$$


(8.6.22) indicates that in regular G.D., $\mathrm{k}-\mathrm{c}_{1}>0$ or $\mathrm{k}>\mathrm{c}_{1}$. Let $A=\Delta-r H+r^{2}$, then

$$
\mathrm{kA}=\mathrm{k} \Delta-\mathrm{rkH}+\mathrm{r}^{2} \mathrm{k},
$$

and using (8.6.18) and (8.6.19), one obtains

$$
\begin{aligned}
k A & =\frac{\lambda_{2} v}{k}\left(a+\lambda_{1}\right)-r\left(a+\lambda_{1}+\lambda_{2} v\right)+k r^{2}, \\
& =\frac{1}{k}\left[\lambda_{2} v\left(r k-r+\lambda_{1}\right)-r k\left(r k-r+\lambda_{1}+\lambda_{2} v\right)+k^{2} r^{2}\right], \\
& =\frac{1}{k}\left(-r \lambda_{2} v+\lambda_{1} \lambda_{2} v+r^{2} k-r k \lambda_{1}\right)=\frac{\left(r-\lambda_{1}\right)\left(r k-\lambda_{2} v\right)}{k} \cdot(8.6 .23)
\end{aligned}
$$

Since $r>\lambda_{1}, r k>\lambda_{2} v$ in regular G.D., one can say that $A=\Delta-r H+r^{2}>0$ always in this class of designs.

Also, from (8.1.11),

$$
\frac{k-c_{1}^{\prime}}{r}=\frac{k}{r}-\frac{c_{1} \Delta-r \lambda_{1}}{r A}
$$

and using (8.6.20) and (8.6.23), one obtains

$$
\begin{aligned}
& \frac{k-c_{1}^{\prime}}{r}=\frac{k}{r}-\frac{\lambda_{1} \lambda_{2} v-k r \lambda_{1}}{\frac{r}{k}\left(r-\lambda_{1}\right)\left(r k-\lambda_{2} v\right)}, \\
& =\frac{k\left(r-\lambda_{1}\right)\left(r k-\lambda_{2} v\right)+k \lambda_{1}\left(r k-\lambda_{2} v\right)}{r\left(r-\lambda_{1}\right)\left(r k-\lambda_{2} v\right)}=\frac{k}{r-\lambda_{1}} \text {. (8.6.24). }
\end{aligned}
$$

Substituting $(8.6 .22)$ and $(8.6 .24)$ into (8.6.16), one can write

$$
V-C=\frac{k}{a+\lambda_{1}} \sigma^{2}+\frac{k}{r-\lambda_{1}} \sigma^{\prime 2}
$$


From (8.6.18),

$$
\frac{n\left(\lambda_{1}-\lambda_{2}\right)}{k \Delta}=\frac{n k\left(\lambda_{1}-\lambda_{2}\right)}{k^{2} \Delta}=\frac{n k\left(\lambda_{1}-\lambda_{2}\right)}{\lambda_{2} v\left(a+\lambda_{1}\right)},
$$

and using $(8.6 .3)$, one obtains

$$
\frac{n\left(\lambda_{1}-\lambda_{2}\right)}{k \Delta}=\frac{k\left(a+\lambda_{1}-\lambda_{2} v\right)}{\lambda_{2} v\left(a+\lambda_{1}\right)}=\frac{k}{\lambda_{2} v}-\frac{k}{a+\lambda_{1}}
$$

From (8.6.23), one can write

$$
\frac{n\left(\lambda_{1}-\lambda_{2}\right)}{k A}=\frac{n k\left(\lambda_{1}-\lambda_{2}\right)}{n k^{2} A}=\frac{n k\left(\lambda_{1}-\lambda_{2}\right)}{\left(r-\lambda_{1}\right)\left(r k-\lambda_{2} v\right)}
$$

Substituting $(8.6 .22),(8.6 .24),(8.6 .26)$, and $(8.6 .27)$ into $(8.6 .17)$,

$$
\begin{aligned}
v-C+n\left(C-C^{\prime}\right) & =\left(\frac{k}{a+\lambda_{1}}+\frac{k}{\lambda_{2} v}-\frac{k}{a+\lambda_{1}}\right) \sigma^{2}+\left[\frac{k}{r-\lambda_{1}}-\frac{n k\left(\lambda_{1}-\lambda_{2}\right)}{\left(r-\lambda_{1}\right)\left(r k-\lambda_{2} v\right)}\right] \sigma^{\prime 2}, \\
& =\frac{k}{\lambda_{2} v} \sigma^{2}+\frac{k\left[r k-\lambda_{2} v-n\left(\lambda_{1}-\lambda_{2}\right)\right]}{\left(r-\lambda_{1}\right)\left(r k-\lambda_{2} v\right)} \sigma^{\prime 2},
\end{aligned}
$$

and by using $(8.6 .3)$, one obtains

$$
\begin{aligned}
v-C+n\left(C-C^{\prime}\right) & =\frac{k}{\lambda_{2} v} \sigma^{2}+\frac{k\left(r-\lambda_{1}\right)}{\left(r-\lambda_{1}\right)\left(r k-\lambda_{2} v\right)} \sigma^{\prime 2}, \\
& =\frac{k}{\lambda_{2} v} \sigma^{2}+\frac{k}{r k-\lambda_{2} v} \sigma^{\prime 2}, \quad(8.6 .28)
\end{aligned}
$$

From (8.6.25) and (8.6.28), one can write: 


$$
\frac{V-C}{v-C+n\left(C-C^{\prime}\right)}=\frac{\frac{k}{a+\lambda_{1}} \sigma^{2}+\frac{k}{r-\lambda_{1}} \sigma^{\prime 2}}{\frac{k}{\lambda_{2} v} \sigma^{2}+\frac{k}{r k-\lambda_{2} v} \sigma^{\prime 2}}
$$

Substituting (8.1.13) into (8.6.29),

$$
\begin{aligned}
\frac{v-c}{v-C+n\left(C-C^{\prime}\right)} & =\frac{\left(\frac{1}{a+\lambda_{1}}+\frac{1}{r-\lambda_{1}}\right) \sigma^{2}+\frac{k}{r-\lambda_{1}} \sigma_{b}^{2}}{\left(\frac{1}{\lambda_{2} v}+\frac{1}{r k-\lambda_{2} v}\right) \sigma^{2}+\frac{k}{r k-\lambda_{2} v} \sigma_{b}^{2}}, \\
& =\frac{\frac{r k}{\left(r-\lambda_{1}\right)\left(a+\lambda_{1}\right)} \sigma^{2}+\frac{k}{r-\lambda_{1}} \sigma_{b}^{2}}{\frac{r k}{\lambda_{2} v\left(r k-\lambda_{2} v\right)} \sigma^{2}+\frac{k}{r k-\lambda_{2} v} \sigma_{b}^{2}}
\end{aligned}
$$

Let $\frac{\sigma_{b}^{2}}{\sigma^{2}}=R>1$, then,

$$
\begin{aligned}
\frac{v-c}{v-c+n\left(C-C^{\prime}\right)} & =\frac{\frac{r}{\left(r-\lambda_{1}\right)\left(a+\lambda_{1}\right)}+\frac{1}{r-\lambda_{1}} R}{\frac{r}{\lambda_{2} v\left(r k-\lambda_{2} v\right)}+\frac{1}{r k-\lambda_{2} v} R}, \\
& =\frac{\alpha_{1}+\beta_{1} R}{\alpha_{2}+\beta_{2}^{R}}=F(R),
\end{aligned}
$$

where

$$
\begin{array}{ll}
\alpha_{1}=\frac{r}{\left(r-\lambda_{1}\right)\left(a+\lambda_{1}\right)}, & \beta_{1}=\frac{1}{r-\lambda_{1}}, \\
\alpha_{2}=\frac{r}{\lambda_{2} v\left(r k-\lambda_{2} v\right)} \quad, & \beta_{2}=\frac{1}{r k-\lambda_{2} v} .
\end{array}
$$




$$
\begin{aligned}
\frac{d F(R)}{d R} & =\frac{\beta_{1}\left(\alpha_{2}+\beta_{2} R\right)-\beta_{2}\left(\alpha_{1}+\beta_{1} R\right)}{\left(\alpha_{2}+\beta_{2} R\right)^{2}} \\
& =\frac{\beta_{1} \alpha_{2}-\beta_{2} \alpha_{1}}{\left(\alpha_{2}+\beta_{2} R\right)^{2}}
\end{aligned}
$$

Substituting from (8.6.31), one can write,

$$
\begin{aligned}
& \beta_{1} \alpha_{2}-\beta_{2} \alpha_{1}=\frac{r}{\lambda_{2} v\left(r-\lambda_{1}\right)\left(r k-\lambda_{2} v\right)}-\frac{r}{\left(r-\lambda_{1}\right)\left(a+\lambda_{1}\right)\left(r k-\lambda_{2} v\right)}, \\
& =\frac{r\left(a+\lambda_{1}\right)-r \lambda_{2} v}{\lambda_{2} v\left(r-\lambda_{1}\right)\left(a+\lambda_{1}\right)\left(r k-\lambda_{2} v\right)}=\frac{r\left(a+\lambda_{1}-\lambda_{2} v\right)}{\lambda_{2} v\left(r-\lambda_{1}\right)\left(a+\lambda_{1}\right)\left(r k-\lambda_{2} v\right)},
\end{aligned}
$$

by $(8.6 .3)$,

$$
=\frac{n r\left(\lambda_{1}-\lambda_{2}\right)}{\lambda_{2} v\left(r-\lambda_{1}\right)\left(a+\lambda_{1}\right)\left(r k-\lambda_{2} v\right)}
$$

Since $r-\lambda_{1}>0, r k-\lambda_{2}>0$ in regular G.D., one can say that

$$
>0 \text { if } \lambda_{1}>\lambda_{2}
$$

$\frac{d F(R)}{d R}$ is

$$
<0 \text { if } \lambda_{1}<\lambda_{2}
$$

Thus, $F(R)$ is monotonically increasing if $\lambda_{1}>\lambda_{2}$, and monotonically decreasing if $\lambda_{1}<\lambda_{2}$. This means that

$$
\begin{aligned}
& F(1)\left\langleF ( R ) \left\langle F(\infty) \text { when } \lambda_{1}>\lambda_{2},\right.\right. \\
& F(\infty)\left\langleF ( R ) \left\langleF ( 1 ) \text { when } \lambda _ { 1 } \left\langle\lambda_{2} .\right.\right.\right.
\end{aligned}
$$


Substituting from (8.6.31),

$$
F(1)=\frac{\alpha_{1}+\beta_{1}}{\alpha_{2}+\beta_{2}}=\frac{\frac{r}{\left(r-\lambda_{1}\right)\left(a+\lambda_{1}\right)}+\frac{1}{\left(r-\lambda_{1}\right)}}{\frac{r}{\lambda_{2} v\left(r k-\lambda_{2} v\right)}+\frac{1}{r k-\lambda_{2} v}}=\frac{\lambda_{2} v\left(r k-\lambda_{2} v\right)\left(r k+\lambda_{1}\right)}{\left(r-\lambda_{1}\right)\left(a+\lambda_{1}\right)\left(r+\lambda_{2} v\right)},
$$

and

$$
F(\infty)=\frac{\beta_{1}}{\beta_{2}}=\frac{r k-\lambda_{2} v}{r-\lambda_{1}} .
$$

For the inverse ratio $G(R)=\frac{1}{F(R)}=\frac{V-C+n\left(C-C^{\prime}\right)}{V-C}$, one has

$$
G(1)=\frac{1}{F(1)}=\frac{\left(r-\lambda_{1}\right)\left(a+\lambda_{1}\right)\left(r+\lambda_{2} v\right)}{\lambda_{2} v\left(r k-\lambda_{2} v\right)\left(r k+\lambda_{1}\right)} \text {, }
$$

and

$$
G(\infty)=\frac{1}{F(\infty)}=\frac{r-\lambda_{1}}{r k-\lambda_{2} v}
$$

Also,

$$
\begin{aligned}
& G(\infty)<G(R)<G(1) \text { if } \lambda_{1}>\lambda_{2}, \\
& G(1)<G(R)<G(\infty) \text { if } \lambda_{1}<\lambda_{2} .
\end{aligned}
$$

\subsubsection{Application of Special Case of Theorem 3}

It should be noticed that the results of the special case are exactly what one desires here, where $\Phi_{1}=\mathrm{V}-\mathrm{C}$, $\Phi_{2}=\mathrm{V}-\mathrm{C}+\mathrm{n}\left(\mathrm{C}-\mathrm{C}^{\prime}\right)$. Let: 


$$
L=\frac{r-\lambda_{1}}{r k-\lambda_{2} v}, \quad P=\frac{\left(r-\lambda_{1}\right)\left(a+\lambda_{1}\right)\left(r+\lambda_{2} v\right)}{\lambda_{2} v\left(r k-\lambda_{2} v\right)\left(r k+\lambda_{1}\right)} .
$$

Then for within comparisons with variance $\Phi_{1}=V-C$, one has $v_{1}=m(n-1)-1, v_{2}=m-1$. Thus for:

$$
\text { (i) } \begin{aligned}
\underline{\lambda_{1}>\lambda_{2}} & : \\
\text { L } & <\frac{\Phi_{2}}{\Phi_{1}}<\mathrm{P},
\end{aligned}
$$

and $G_{2}$ in $(8.5 .7)$ becomes:

$$
G_{2}=\int_{0}^{1} \frac{u^{\frac{1}{2}(v-m-3)}(1-u)^{\frac{1}{2}(m-3)}}{[u+L(1-u)]^{2}} d u .
$$

Also $G_{1}$ in $(8.5 .8)$ becomes:

$$
G_{1}=\int_{0}^{1} \frac{u^{\frac{1}{2}(v-m-3)}(1-u)^{\frac{1}{2}(m-3)}}{u+P(1-u)} d u \text {. }
$$

And for:

$$
\text { (ii) } \begin{aligned}
\frac{\lambda_{1}<\lambda_{2}}{} & \\
P & <\frac{\Phi_{2}}{\Phi_{1}}<L \quad \text {; }
\end{aligned}
$$

$G_{2}$ and $G_{1}$ in $(8.5 .7)$ and $(8.5 .8)$ become:

$$
G_{2}=\int_{0}^{1} \frac{u^{\frac{1}{2}(v-m-3)}(1-u)^{\frac{1}{2}(m-3)}}{[u+P(1-u)]^{2}} d u,
$$


and

$$
G_{1}=\int_{0}^{1} \frac{u^{\frac{1}{2}(v-m-3)}(1-u)^{\frac{1}{2}(m-3)}}{u+L(1-u)} d u
$$

For among comparisons with variance $\Phi_{2}=\mathrm{V}-\mathrm{C}+\mathrm{n}\left(\mathrm{C}-\mathrm{C}^{\prime}\right)$, one has $v_{1}=m(n-1), v_{2}=m-2$, and for:

$$
\text { (i) } \begin{aligned}
\frac{\lambda_{1}>\lambda_{2}}{2} & \\
\frac{1}{\mathrm{P}} & <\frac{\Phi_{1}}{\Phi_{2}}<\frac{1}{\mathrm{~L}} \text {; }
\end{aligned}
$$

$\mathrm{G}_{2}^{\prime}$ in $(8.5 .14)$ becomes:

$$
\begin{aligned}
G_{2}^{\prime} & =\int_{0}^{1} \frac{u^{\frac{1}{2}(v-m-2)}(1-u)^{\frac{1}{2}(m-4)}}{\left[\frac{1}{P} u+(1-u)\right]^{2}} d u, \\
& =P^{2} \int_{0}^{1} \frac{u^{\frac{1}{2}(v-m-2)}(1-u)^{\frac{1}{2}(m-4)}}{[u+P(1-u)]^{2}} d u
\end{aligned}
$$

$G_{1}^{\prime}$ in $(8.5 .15)$ becomes:

$$
\begin{aligned}
G_{1}^{\prime} & =\int_{0}^{1} \frac{u^{\frac{1}{2}(v-m-2)}(1-u)^{\frac{1}{2}(m-4)}}{\frac{1}{I} u+(1-u)} d u, \\
& =I \int_{0}^{1} \frac{u^{\frac{1}{2}(v-m-2)}(1-u)^{\frac{1}{2}(m-4)}}{u+I(1-u)} d u
\end{aligned}
$$


And for:

$$
\begin{aligned}
& \text { (ii) } \lambda_{1}<\lambda_{2} \text { : } \\
& \frac{1}{L}<\frac{\Phi_{1}}{\Phi_{2}}<\frac{1}{P} \quad \text {; }
\end{aligned}
$$

$\mathrm{G}_{2}^{\prime}$ and $\mathrm{G}_{1}^{\prime}$ become, in this case:

$$
\begin{aligned}
G_{2}^{\prime} & =\int_{0}^{1} \frac{u^{\frac{1}{2}(v-m-2)}(1-u)^{\frac{1}{2}(m-4)}}{\left[\frac{1}{L} u+(1-u)\right]^{2}} d u \\
& =L^{2} \int_{0}^{1} \frac{u^{\frac{1}{2}(v-m-2)}(1-u)^{\frac{1}{2}(m-4)}}{[u+u(1-u)]^{2}} d u
\end{aligned}
$$

and

$$
\begin{aligned}
G_{1}^{\prime} & =\int_{0}^{1} \frac{u^{\frac{1}{2}(v-m-2)}(1-u)^{\frac{1}{2}(m-4)}}{\frac{1}{P} u+(1-u)} d u \\
& =P \int_{0}^{1} \frac{u^{\frac{1}{2}(v-m-2)}(1-u)^{\frac{1}{2}(m-4)}}{u+P(1-u)} d u
\end{aligned}
$$

One notices that the integrals for the within comparisons can be evaluated for $m \geq 2$, but for among comparisons one must have $\mathrm{m}>2$. The combining constant $\mathrm{B}$ and a conservative lower bound of the recovery ratio $D$ are given in (8.5.12) and (8.5.20) for the within comparisons; and for the among comparisons $B$ and $D$ are given in (8.5.18) and (8.5.22), respectively. The required integrals have been evaluated 
and the corresponding values for $B$ and $D$ have been computed for 62 regular G.D. designs that appear in Bose's "Tables for P.B.I.B". The results are listed in Tables III and IV for the within and among comparisons respectively. The combined estimate, as given in $(8.4 .1)$ is

$$
\hat{\tau}_{i}=U_{i}+\frac{\theta_{i} B s^{2}}{\sum_{\substack{j=1 \\ j \neq i}}^{v-1}\left(x_{j}-U_{j}\right)^{2}}\left(x_{i}-U_{i}\right),
$$

where $\theta_{i}$ is the coefficient of $\sigma^{2}$ in $V\left(U_{i}\right)$.

For within comparisons, one has, using (8.2.5) and $(8.6 .22):$

$$
\mathrm{V}\left(\mathrm{U}_{i}\right)=\mathrm{V}_{1}-\mathrm{C}_{1}=\frac{\mathrm{k}-\mathrm{C}_{1}}{\mathrm{a}} \sigma^{2}=\frac{\mathrm{k}}{\mathrm{a}+\lambda_{1}} \sigma^{2},
$$

hence $\theta_{i}=\frac{k}{a+\lambda_{1}}$ and the combined estimate in (8.6.52) becomes:

$$
\hat{\tau}_{i}=U_{i}+\frac{\mathrm{kBS}^{2}}{\left(a+\lambda_{1}\right) \sum_{\substack{j=1 \\ j \neq i}}^{v-1}\left(x_{j}-U_{j}\right)^{2}}\left(x_{i}-U_{i}\right) \cdot(8.6 .54)
$$

For among comparisons, one has, using (8.6.8), (8.2.5), $(8.3 .1),(8.6 .22)$, and $(8.6 .26):$ 


$$
\begin{aligned}
V\left(U_{i}\right) & =v_{1}-c_{1}+n\left(c_{1}-c_{2}\right)=\left(\frac{k-c_{1}}{a}+\frac{n\left(\lambda_{1}-\lambda_{2}\right)}{k \Delta}\right) \sigma^{2}, \\
& =\left(\frac{k}{a+\lambda_{1}}+\frac{k}{\lambda_{2} v}-\frac{k}{a+\lambda_{1}}\right) \sigma^{2}=\frac{k}{\lambda_{2} v} \sigma^{2},
\end{aligned}
$$

hence $\theta_{i}=\frac{k}{\lambda_{2} v}$ and the combined estimate in (8.6.52) becomes:

$$
\hat{\tau}_{i}=u_{i}+\frac{k B s^{2}}{\lambda_{2} v \sum_{\substack{j=1 \\ j \neq i}}^{v-1}\left(x_{j}-U_{j}\right)^{2}}\left(x_{i}-U_{i}\right)
$$

The $B^{\prime}$ 's in (8.6.54) and (8.6.56) are the combining constants to be obtained from Tables III and IV, respectively.

\subsubsection{Analysis for Singular and Semi-regular G.D. Designs}

The formula (8.1.9) of M. Zelen for the inter-block estimate is not applicable in the two subclasses, singular G.D., and semi-regular G.D. designs because the quantity $\Delta-r H+r^{2}=0$ for both. An inter-analysis for those subclasses will now be given.

It is known that in the inter-block analysis it is assumed that the block effects $b_{s}$ are random variables, uncorrelated with each other and with the plot errors $\varepsilon_{i j s}$ 's, having mean zero and (unknown) variance $\sigma_{b}^{2}$. Let the total 
of blocks be denoted by $B_{s}, s=1,2, \ldots, b$, and the effect of the treatment in the i-th row and $j-t h$ column in the association scheme rectangle by $t_{i j}$, where $i=1,2, \ldots, m, j=1,2, \ldots, n$, $\mathrm{mn}=\mathrm{v}$. One can then express the yield $\mathrm{y}_{\mathrm{ijs}}$ as:

$$
y_{i j s}=\mu+t_{i j}+b_{s}+\varepsilon_{i j s},
$$

where $y_{i j s}$ is defined only when the treatment (ij) occurs in the block s. The total of the $s-t h$ block is:

$$
B_{s}=k \mu+\sum_{i=1}^{m} \sum_{j=1}^{n} \delta_{i j s} t_{i j}+k b_{s}+\sum_{i=1}^{m} \sum_{j=1}^{n} \delta_{i j s} \varepsilon_{i j s},
$$

where

$$
\begin{aligned}
& 1 \text { if }(i j)-t h \text { treatment occurs in s-th block, } \\
& \delta_{i j s}=0 \text { otherwise. }
\end{aligned}
$$

Here $\mathrm{E}\left(\mathrm{B}_{\mathrm{S}}\right)=\mathrm{k} \mu+\sum_{i j} \delta_{i j \mathrm{~s}} \mathrm{t}_{i j}$ and $\mathrm{V}\left(\mathrm{B}_{\mathrm{S}}\right)=\mathrm{k}\left(\sigma^{2}+\mathrm{k} \sigma_{\mathrm{b}}^{2}\right)$. One obtains the normal equations by minimizing the quantity,

$$
\sum_{s=1}^{b}\left(B_{s}-k \mu-\sum_{i}^{m} \sum_{j}^{n} \delta_{i j s} t_{i j}\right)^{2}-\theta \sum_{i j} t_{i j} \text {. }
$$

The normal equations, therefore, are:

$$
\begin{gathered}
-2 k \sum_{s=1}^{b}\left(B_{s}-k \hat{\mu}-\sum_{i j} \delta_{i j s} \hat{t}_{i j}^{\prime}\right)=0, \\
-\sum_{s=1}^{b} \delta_{i j s}\left(B_{s}-k \hat{\mu}-\sum_{i} \delta_{j \prime} \delta_{i ' j ' s} \hat{t}_{i}^{\prime} j^{\prime}{ }^{\prime}\right)-\theta=0 .
\end{gathered}
$$


Noticing that $\sum_{s=1}^{b} \delta_{i j s}=r, \sum_{i=1}^{m} \sum_{j=1}^{n} \delta_{i j s}=k$, and simplifying $(8.6 .60)$, and $(8.6 .61)$, one can write

$$
\begin{gathered}
b k^{2} \hat{\mu}+k \sum_{s=1}^{b} \sum_{i j} \delta_{i j s} \hat{t}_{i j}^{\prime}=k G, \\
k r \hat{\mu}+\sum_{i j^{\prime}}\left(\sum_{s} \delta_{i j s^{\prime} \delta^{\prime} j^{\prime} s^{\prime}}\right) \hat{t}_{i^{\prime} j^{\prime}}=\sum_{s=1}^{b} \delta_{i j s^{B} s}+\theta .
\end{gathered}
$$

Let $\sum_{\mathrm{s} i j} \delta_{i j \mathrm{~s}} \hat{t}_{i j}^{\prime}=0$, then (8.6.62) gives $\hat{\mu}=\frac{\mathrm{G}}{\mathrm{bk}}$, and substituting in (8.6.63) one obtains:

$$
\sum_{i^{\prime} j^{\prime}}\left(\sum_{s} \delta_{i j s^{\prime}} \delta_{i^{\prime} j^{\prime} s}\right) \hat{t}_{i^{\prime} j}^{\prime}=B_{i j} .-\frac{r G}{b}+\theta=Q_{i j}^{\prime}+\theta \text {, }
$$

where $B_{i j .}=\sum_{s=1}^{b} \delta_{i j s}{ }^{B} s=$ the sum of block totals in which treatment ( $i j)$ occurs. If $i j=i^{\prime} j^{\prime}$ then $\sum_{s} \delta_{i j s} \delta_{i \prime j ' s}=\sum_{s} \delta_{i j s}^{2}=\sum_{s} \delta_{i j s}=r$, thus (8.6.64) becomes

$$
r \hat{t}_{i j}^{\prime}+\sum_{i j^{\prime} \neq i j}\left(\sum_{s} \delta_{i j s} \delta_{i \prime j ' s}\right) \hat{t}_{i j^{\prime}}^{\prime}=Q_{i j}^{\prime}+\theta,
$$

or

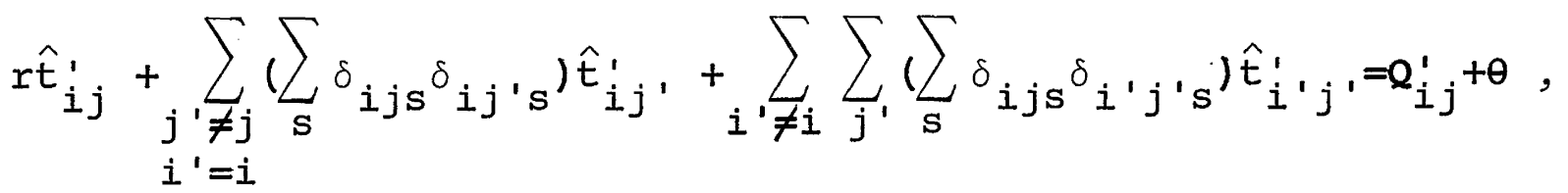


but,

$\sum_{s} \delta_{i j s} \delta_{i j ' s}=$ the number of blocks in which treatment

(ij) and treatment $\left(i j^{\prime}\right)$, where $j \neq j^{\prime}$ occur together $=\lambda_{1}$. Also,

$\sum_{s} \delta_{i j s} \delta_{i ' j ' s}=$ the number of blocks in which treatment

(ij) and treatment $\left(i^{\prime} j^{\prime}\right)$ occur together, where $i \neq i^{\prime}=\lambda_{2}$. Hence (8.6.65) becomes:

$$
r \hat{t}_{i j}^{\prime}+\sum_{j^{\prime}=j} \lambda_{1} \hat{t}_{i j^{\prime}}^{\prime}+\sum_{i \neq j} \sum_{j^{\prime}} \lambda_{2} \hat{t}_{i^{\prime} j^{\prime}}=Q_{i j}^{\prime}+\theta \cdot(8.6 .66)
$$

Summing over all treatments one obtains:

$r$ (sum of all treatments) $+n_{1} \lambda_{1}$ (sum of all treatments)

$$
+\mathrm{n}_{2} \lambda_{2} \text { (sum of all treatments) }=0+v \theta \text {, }
$$

i.e., $v \theta=0$ or $\theta=0$. Using again the assumption that the sum of all treatments $=0$, one can write:

$$
\sum_{i \neq \neq i} \sum_{j^{\prime}} \hat{t}_{i^{\prime} j^{\prime}}=-\sum_{j} \hat{t}_{i j^{\prime}},
$$

i.e., the sum of all treatments except the $i-t h$ row $=$ - the sum of the i-th row. Hence (8.6.66) takes the following form for all treatments in the i-th row:

$$
r \hat{t}_{i j}^{\prime}+\lambda_{1} \sum_{j \neq j} \hat{t}_{i j^{\prime}}^{\prime}-\lambda_{2} \sum_{j} \hat{t}_{i j^{\prime}}=Q_{i j}^{\prime}, \quad j=1,2, \ldots, n,
$$


or

$$
\left(r-\lambda_{2}\right) \hat{t}_{i j}^{\prime}+\left(\lambda_{1}-\lambda_{2}\right) \sum_{j \prime \neq j} \hat{t}_{i j}^{\prime}=Q_{i j}^{\prime}, \quad j=1,2, \ldots, n .
$$

Now there are m equations similar to (8.6.69) for

$i=1,2,3 \ldots m$. Equation (8.6.69) becomes in matrix notation, for $j=1,2, \ldots, n$ :

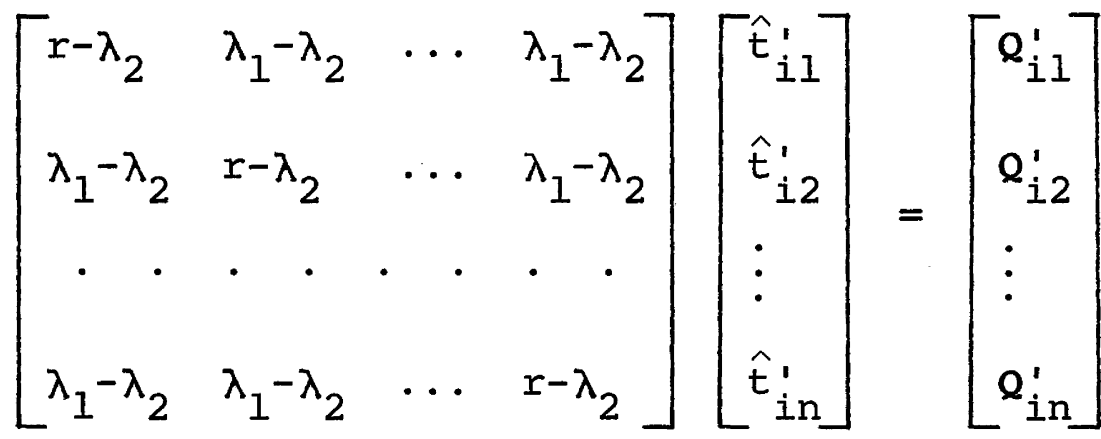

8.6.2.1 Inter-estimates and Recovery of Inter-information in. Singular G.D.

In this case $r=\lambda_{1}$ and the coefficient matrix in (8.6.70) is of rank one. Out of each group or row of treatments, one can in fact obtain an inter-estimate of one selected treatment and impose arbitrary values for the remaining $n-1$ treatments. This is natural, due to the fact that one can construct singular G.D. designs by stretching every treatment in a B.I.B. design to become a group of $n$ treatments or a row in the association scheme. Assume that 
n-1 treatments in each row have the estimate zero. Then one can write

$$
\hat{t}_{i j}^{\prime}=\frac{Q_{i j}^{\prime}}{\lambda_{1}-\lambda_{2}} \quad i=1,2,3 \ldots m,
$$

and $\hat{t}_{i j^{\prime}}=0$ for $j^{\prime}=1,2, \ldots, j-1, j+1, j+2, \ldots, n$.

In this way one has inter-estimates for $m$ treatments, one from each row. It should be noted that these estimates must sum to zero, and that each treatment is a second associate of all the remaining m-1 treatments. It is obvious that one can inter-estimate m-l comparisons; we shall choose the $\mathrm{m}-1$ among comparisons defined in section 8.6.1 to be inter- and intra-estimated. They have the same variance, as it was shown in $(8.6 .8)$, so that theorem 1 is applicable. From formula (3.1), where $\mathrm{m}$ is $\mathrm{m}-1$, one obtains:

$$
\hat{\tau}_{i}=U_{i}+\frac{\theta(m-3) s^{2}}{\sum_{j=1}^{m-1}\left(x_{j}-U_{j}\right)^{2}}\left(x_{i}-U_{i}\right) .
$$

This combined estimate is applicable for $m 3 . \cdot \theta=\frac{p f}{f+2}$, $\rho$ is the coefficient of $\sigma^{2}$ in $V\left(U_{i}\right)=v_{1}-C_{1}+n\left(C_{1}-C_{2}\right)=\frac{k}{\lambda_{2} v} \sigma^{2}$, as was shown before in $(8.6 .55)$. Hence $\rho=\frac{\mathrm{k}}{\lambda_{2} \mathrm{v}}$, and then 
(8.6.72) becomes:

$$
\hat{\tau}_{i}=U_{i}+\frac{f k(m-3) s^{2}}{(f+2) \lambda_{2} v \sum_{j=1}^{m-1}\left(x_{j}-U_{j}\right)^{2}}\left(x_{i}-U_{i}\right) .
$$

The recovery ratio, from theorem 1, is then:

$$
D=\frac{f}{(2+f)(m-1)} \frac{(m-3)}{(m-1)}
$$

where $f=r v-v-b+1$.

The choice of the among comparisons is justified here by the fact that the efficiency $\mathbf{E}$ of any comparison between first associate treatments is unity, as R. C. Bose's tables for P.B.I.B. indicate.

\subsubsection{Inter-estimates and Recovery of Inter-block Infor-} mation in Semi-Regular G.D.

In this case $r k=\lambda_{2} v$, and by $(8.6 .3)$ one can write:

$$
r-\lambda_{1}+n\left(\lambda_{1}-\lambda_{2}\right)=0 \text {, }
$$

or

$$
\left(r-\lambda_{1}\right) * \lambda_{1}-\lambda_{2} * n_{1}\left(\lambda_{1}-\lambda_{2}\right)=0 \text {, where } n_{1}=n-1 \text {, }
$$

or

$$
r-\lambda_{2}=-n_{1}\left(\lambda_{1}-\lambda_{2}\right)
$$


The matrix equation in $(8.6 .70)$ becomes:

$$
\left[\begin{array}{cccc}
-n_{1}\left(\lambda_{1}-\lambda_{2}\right) & \lambda_{1}-\lambda_{2} & \ldots & \lambda_{1}-\lambda_{2} \\
\lambda_{1}-\lambda_{2} & -n_{1}\left(\lambda_{1}-\lambda_{2}\right) & \ldots & \lambda_{1}-\lambda_{2} \\
\lambda_{1}-\lambda_{2} & \lambda_{1}-\lambda_{2} & \ldots & -n_{1}\left(\lambda_{1}-\lambda_{2}\right)
\end{array}\right]\left[\begin{array}{c}
\hat{t}_{i 1}^{\prime} \\
\hat{t}_{i 2}^{\prime} \\
\vdots \\
\hat{t}_{i n}^{\prime}
\end{array}\right]=\left[\begin{array}{c}
Q_{i 1}^{\prime} \\
Q_{i 2}^{\prime} \\
\vdots \\
Q_{i n}^{\prime}
\end{array}\right] .
$$

The coefficient matrix in $(8.6 .76)$ is of rank $n-1$, i.e., out of each group or row of treatments, one can in fact interestimate n-1 treatments and impose arbitrary value for the remaining one. Applying the restriction $\sum_{j=1}^{n} \hat{t}_{i j}^{\prime}=0$, and using (8.6.75) one can write $(8.6 .69)$ as:

$$
\begin{aligned}
& -(n-1)\left(\lambda_{1}-\lambda_{2}\right) \hat{t}_{i j}^{\prime}+\left(\lambda_{1}-\lambda_{2}\right) \sum_{j \neq j} \hat{t}_{i j}^{\prime}=Q_{i j}^{\prime}, \text { or } \\
& -n\left(\lambda_{1}-\lambda_{2}\right) \hat{t}_{i j}^{\prime}+\left(\lambda_{1}-\lambda_{2}\right) \sum_{j=1}^{n} \hat{t}_{i j}^{\prime}=Q_{i j}^{\prime}, \text { or } \\
& -n\left(\lambda_{1}-\lambda_{2}\right) \hat{t}_{i j}^{\prime}=Q_{i j}^{\prime}, \quad i . e ., \\
& \hat{t}_{i j}^{\prime}=\frac{Q_{i j}^{\prime}}{n\left(\lambda_{2}-\lambda_{1}\right)}=\frac{Q_{i j}^{\prime}}{r-\lambda_{1}}, \\
& i=1,2, \ldots, m ; \quad j=1,2,3 \ldots n-1 .
\end{aligned}
$$


It is obvious that one can inter-estimate n-I comparisons within each row of treatments; in all one can interestimate $m(n-1)$ comparisons. shall choose the $m(n-1)$ within comparisons defined in Section 8.6 .1 to be inter- and intra-estimated. They have the same variance, namely $\mathrm{V}_{1}-\mathrm{C}_{1}$ for intra-estimate and $V_{1}^{\prime}-C_{1}^{\prime}$ for inter-estimate, so that theorem 1 is applicable. This gives the following combined estimate:

$$
\hat{\tau}_{i}=U_{i}+\frac{\theta(m n-m-2) s^{2}}{m\left(\sum_{j=1}^{(n-1)}\left(x_{j}-U_{j}\right)^{2}\right.}\left(x_{i}-U_{i}\right) .
$$

This combined estimate is applicable for $\mathrm{mn}-\mathrm{m}-2=\mathrm{v}-\mathrm{m}-2>0$ or $v-m>2 . \quad \theta=\frac{\rho f}{f+2}, \rho$ is the coefficient of $\sigma^{2}$ in $\mathrm{V}\left(\mathrm{U}_{\mathrm{i}}\right)=\mathrm{V}_{1}-\mathrm{C}_{1}=\frac{\mathrm{k}-\mathrm{C}_{1}}{\mathrm{a}} \sigma^{2}=\frac{\mathrm{k}}{\mathrm{a}+\lambda_{1}} \sigma^{2}$, as it was shown in (8.6.53). Hence $\rho=\frac{k}{a+\lambda_{1}}$, and then (8.6.78) becomes

$$
\hat{\tau}_{i}=u_{i}+\frac{k(v-m-2) s^{2}}{v-m}\left(x_{i}-U_{i}\right) \frac{f}{f+2} .
$$

The recovery ratio is,

$$
D=-\frac{f}{(2+f)(v-m)} \frac{(v-m-2)}{,}
$$

where $f=r v-v-b+1$. 
The choice of the within comparisons is justified by the fact that the efficiency $E_{1}$ of a comparison between first associate treatments is always less than $\mathbf{E}_{2}$, the efficiency of a comparison between second associate treatments, as R. C. Bose's tables for P.B.I.B. indicate.

\subsection{Recovery of Inter-block Information in LS Type}

In this case $\mathrm{v}=\mathrm{n}^{2}$, and the treatments can be arranged in an nxn square association scheme so that two treatments are first associates if they occur together in the same row or in the same column, and they are second associates otherwise. Such a design will be said to belong to the sub-type $\mathrm{I}_{2}$ of the Latin Square type design denoted by LS. We also have designs with $\mathrm{n}^{2}$ treatments belonging to the sub-type $\mathrm{L}_{3}$ of the Latin Square type design. In this case it is possible to form an nxn square array and to impose a Latin Square with $\mathrm{n}$ letters on this array, so that any two treatments are first associates if they occur in the same row or column of the array or correspond to the same letter, and are second associates otherwise.

The following relations hold for the sub-type $I_{i}$ of LS designs $(i=2,3)$. 


$$
\begin{gathered}
n_{1}=i(n-1), \quad n_{2}=(n-1)(n-i+1), \\
P_{1}=\left(\begin{array}{cc}
i^{2}-3 i+n & (i-1)(n-i+1) \\
(i-1)(n-i+1) & (n-i)(n-i+1)
\end{array}\right), \\
P_{2}=\left(\begin{array}{cc}
i(i-1) & i(n-i) \\
i(n-1) & (n-i)^{2}+i-2
\end{array}\right)
\end{gathered}
$$

Consider now the following $\mathrm{n}^{2} \times \mathrm{n}^{2}$ matrix $\mathrm{M}_{1}$, 


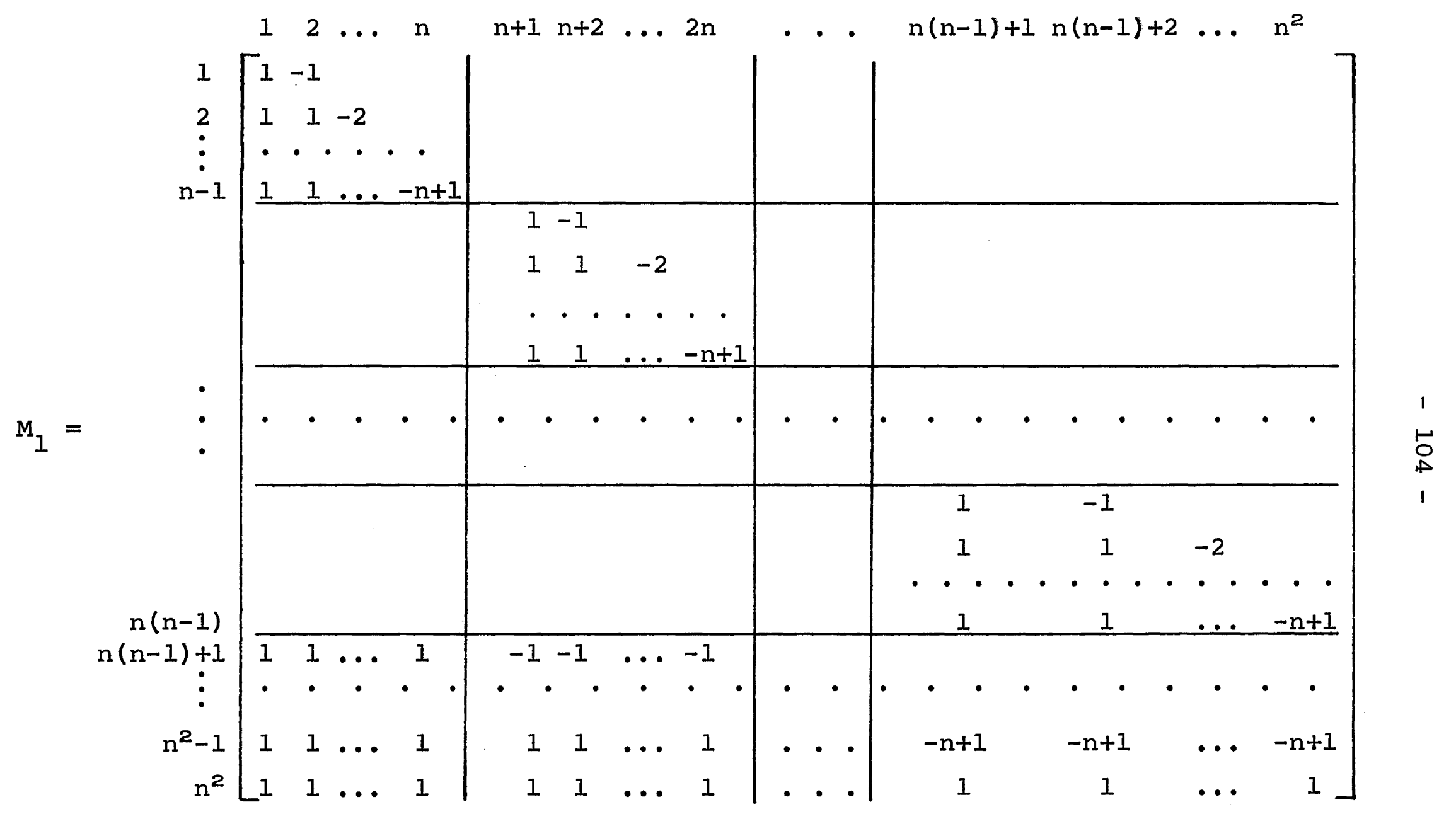


Let $M$ be the matrix $M_{1}$ after normalizing its rows, then $M$ is orthogonal. Define:

$$
\underline{U}=\left[\begin{array}{l}
U_{1} \\
\vdots \\
u_{n(n-1)} \\
\cdots \cdots \cdots \\
u_{n(n-1)+1} \\
\vdots \\
u_{n^{2}-1} \\
0
\end{array}\right]=M\left[\begin{array}{c}
\hat{t}_{1} \\
\hat{t}_{2} \\
\vdots \\
\\
\hat{t}_{n^{2}}
\end{array}\right]=M \underline{\underline{t}} .
$$

Also define

$$
\underline{x}=\left[\begin{array}{l}
x_{1} \\
\vdots \\
x_{n(n-1)} \\
\cdots \cdots \cdots \\
x_{n(n-1)+1} \\
\vdots \\
x_{n^{2}-1} \\
0
\end{array}\right]=M\left[\begin{array}{c}
\hat{t}_{1}^{\prime} \\
\hat{t}_{2}^{\prime} \\
\vdots \\
\\
\hat{t}_{n^{2}}^{\prime}
\end{array}\right]=M \underline{\hat{t}}^{\prime},
$$

where $\hat{t}_{i}$ and $\hat{t}_{i}^{\prime}$ are the intra- and inter-estimates of the $i-t h$ treatment effect, respectively. The treatments in $\hat{\underline{t}}$ or $\underline{\hat{E}}^{\prime}$ are arranged according to the rows of the association scheme, i.e., the first $\mathrm{n}$ are the treatments of the first row, the next $\mathrm{n}$ treatments are those of the second row, etc. 
Since the treatments in the same group (or row) are first associates, we have in similarity with (7.2.4) that:

$$
\mathrm{V}\left(\mathrm{U}_{j}\right)=\mathrm{v}_{1}-\mathrm{C}_{1}, \text { for } j=1,2, \ldots, \mathrm{n}(\mathrm{n}-1) \text {. }
$$

Thus all within comparisons have the same variance, namely $\mathrm{V}_{1}-\mathrm{C}_{1}$. For the among comparisons, let us compute for $\mathrm{L}_{i}$ sub-type $(i=2,3)$ :

$$
\begin{aligned}
& v\left(U_{n(n-1)+m}\right)=v\left\{\frac { 1 } { \sqrt { n m ( m + 1 ) } } \left[\left(\hat{t}_{1}+\ldots+\hat{t}_{n}\right)+\left(\hat{t}_{n+1}+\ldots+\hat{t}_{2 n}\right)\right.\right. \\
& \left.\left.+\ldots-m\left(t_{n m+1}+\ldots+t_{n(m+1)}\right)\right]\right\}, \\
& =\frac{1}{n m(m+1)}\left\{n m(m+1) v_{1}+n(n-1) m c_{1}+m(m-1)\left[n\left(c_{1}(i-1)+c_{2}(n-i+1)\right)\right]\right. \\
& \left.+n(n-1) m^{2} c_{1}-2 m\left[n\left((i-1) m c_{1}+m(n-i+1) c_{2}\right)\right]\right\} \text {, } \\
& =v_{1}+\frac{1}{n m(m+1)} c_{1}\left[n(n-1) m+n(i-1) m(m-1)+n(n-1) m^{2}-2 n m^{2}(i-1)\right] \\
& +\frac{1}{n m(m+1)} c_{2}\left[n m(m-1)(n-i+1)-2 m^{2} n(n-i+1)\right], \\
& =v_{1}+\frac{n m C_{1}}{n m(m+1)}[n-1+(i-1)(m-1)+m(n-1)-2 m(i-1)] \\
& +\frac{C_{2} n m(n-i+1)}{n m(m+1)}(m-1-2 m) \text {, } \\
& =v_{1}+\frac{C_{1}}{m+1}[(n-1)(m+1)+(i-1)(m-1-2 m)]-C_{2}(n-i+1), \\
& =v_{1}+c_{1}(n-1-i+1)-c_{2}(n-i+1), \\
& =v_{1}-c_{1}+(n-i+1)\left(c_{1}-c_{2}\right) \text {. }
\end{aligned}
$$


This is independent of $\mathrm{m}$, which indicates that all among comparisons have the same variance, namely:

$$
\begin{aligned}
V\left(U_{j}\right)=v_{1}-C_{1} & +(n-i+1)\left(c_{1}-c_{2}\right), \\
& \text { where } j=n(n-1)+1, \ldots, n^{2}-1,
\end{aligned}
$$

and $(i=2,3)$ according to the design being of $L_{2}$ or $L_{3}$ subtypes.

The inter-variances are similarly:

$$
\begin{gathered}
v\left(x_{j}\right)=v_{1}^{\prime}-c_{1}^{\prime}, \quad i=1,2, \ldots, n(n-I), \\
v\left(x_{j}\right)=v_{1}^{\prime}-c_{1}^{\prime}+(n-i+1)\left(c_{1}^{\prime}-c_{2}^{\prime}\right), \\
j=n(n-1)+1, \ldots, n^{2}-1,
\end{gathered}
$$

and $(i=2,3)$.

Let:

$$
\underline{\mathrm{Z}}=\underline{\mathrm{X}}-\underline{\mathrm{U}}
$$

Then,

$$
\begin{aligned}
\mathrm{v}\left(\mathrm{z}_{j}\right) & =\mathrm{v}\left(\mathrm{x}_{j}\right)+\mathrm{v}\left(\mathrm{U}_{j}\right), \\
& =\mathrm{v}_{1}^{\prime}-\mathrm{C}_{1}^{\prime}+\mathrm{v}_{1}-\mathrm{C}_{1}=\mathrm{v}-\mathrm{C}, \quad j=1,2 \ldots \mathrm{n}(\mathrm{n}-1),
\end{aligned}
$$

and

$$
\begin{aligned}
V\left(z_{j}\right) & =v_{1}^{\prime}-c_{1}^{\prime}+(n-i+1)\left(c_{1}^{\prime}-c_{2}^{\prime}\right)+v_{1}-c_{1}+(n-i+1)\left(c_{1}-c_{2}\right), \\
& =v-c+(n-i+1)\left(c-c^{\prime}\right), \quad j=n(n-1)+1, \ldots, n^{2}-1,
\end{aligned}
$$

where $\mathrm{V}, \mathrm{C}, \mathrm{C}^{\prime}$ are as defined in (8.2.15) through (8.2.17). 
As it was shown in $(8.6 .16)$, one can write in this case:

$$
\mathrm{V}-\mathrm{C}=\frac{\mathrm{k}-\mathrm{C}_{1}}{\mathrm{a}} \sigma^{2}+\frac{\mathrm{k}-\mathrm{C}_{1}^{\prime}}{\mathrm{r}} \sigma^{\prime 2} \text {. }
$$

By $(8.3 .5)$ we can write:

$$
\begin{aligned}
V-C+(n-i+1)\left(C-C^{\prime}\right)= & {\left[\frac{k-c_{1}}{a}+\frac{(n-i+1)\left(\lambda_{1}-\lambda_{2}\right)}{k \Delta}\right] \sigma^{2} } \\
& +\left[\frac{k-c_{1}^{\prime}}{r}+\frac{(n-i+1)\left(\lambda_{1}-\lambda_{2}\right)}{k\left(\Delta-r H+r^{2}\right)}\right] \sigma^{\prime 2} .
\end{aligned}
$$

One notices that the special case of theorem 3 is applicable here where $\Phi_{1}=V-C, \Phi_{2}=V-C+(n-i+1)\left(C-C^{\prime}\right)$, and

$$
\frac{\Phi_{1}}{\Phi_{2}}=\frac{\alpha_{1} \sigma^{2}+\alpha_{2} \sigma^{\prime 2}}{\left(\alpha_{1}+\beta_{1}\right) \sigma^{2}+\left(\alpha_{2}+\beta_{2}\right) \sigma^{\prime 2}}
$$

Substituting for $\sigma^{\prime 2}$ from (8.1.13), one obtains

$$
\frac{\Phi_{1}}{\Phi_{2}}=\frac{\left(\alpha_{1}+\alpha_{2}\right) \sigma^{2}+k \alpha_{2} \sigma_{b}^{2}}{\left(\alpha_{1}+\beta_{1}+\alpha_{2}+\beta_{2}\right) \sigma^{2}+k\left(\alpha_{2}+\beta_{2}\right) \sigma_{b}^{2}}
$$

where:

$$
\begin{array}{ll}
\alpha_{1}=\frac{k-c_{1}}{a}, & \alpha_{2}=\frac{k-c_{1}^{\prime}}{r}, \\
\beta_{1}=\frac{(n-i+1)\left(\lambda_{1}-\lambda_{2}\right)}{k \Delta}, & \beta_{2}=\frac{-(n-i+1)\left(\lambda_{1}-\lambda_{2}\right)}{k\left(\Delta-r H+r^{2}\right)} .
\end{array}
$$


Remembering that $\frac{\sigma_{b}^{2}}{\sigma^{2}}=R>1$, one can write $(8.7 .15)$ as:

$$
\begin{aligned}
& \frac{\Phi_{1}}{\Phi_{2}}=F(R)=\frac{\left(\alpha_{1}+\alpha_{2}\right)+k \alpha_{2} R}{\left(\alpha_{1}+\beta_{1}+\alpha_{2}+\beta_{2}\right)+k\left(\alpha_{2}+\beta_{2}\right) R}, \\
& \frac{d F(R)}{d R}=\frac{k\left(\beta_{1} \alpha_{2}-\alpha_{1} \beta_{2}\right)}{\left[\left(\alpha_{1}+\beta_{1}+\alpha_{2}+\beta_{2}\right)+k\left(\alpha_{2}+\beta_{2}\right) R\right]^{2}},
\end{aligned}
$$

For the LS type designs listed in Bose's tables $\alpha_{1}, \alpha_{2}$, $\Delta$, and $\left(\Delta-r H+r^{2}\right)$ are all positive, hence the sign of $\frac{d F(R)}{d R}$ is the same as the sign of $\left(\beta_{1} \alpha_{2}-\alpha_{1} \beta_{2}\right)$.

Substituting $\beta_{1}, \alpha_{1}, \beta_{2}, \alpha_{2}$ from $(8.7 .16)$, one obtains

$$
\begin{aligned}
\beta_{1} \alpha_{2}-\alpha_{1} \beta_{2} & =\left(\lambda_{1}-\lambda_{2}\right)\left[\frac{(n-i+1) \alpha_{2}}{k \Delta}+\frac{(n-i+1) \alpha_{1}}{k\left(\Delta-r H+r^{2}\right)}\right] \\
& =\left(\lambda_{1}-\lambda_{2}\right) \times \text { (positive quantity) } .
\end{aligned}
$$

Thus, the sign of $\frac{d F(R)}{d R}$ is the same as that of $\lambda_{1}-\lambda_{2}$, i.e.,

$$
\begin{aligned}
& F(R) \text { is monotonically increasing if } \lambda_{1}>\lambda_{2}, \\
& F(R) \text { is monotonically decreasing if } \lambda_{1}<\lambda_{2} .
\end{aligned}
$$

Consequently one can write:

$$
\begin{aligned}
& F(1)<F(R)<F(\infty) \text { when } \lambda_{1}>\lambda_{2}, \\
& F(\infty)<F(R)<F(1) \text { when } \lambda_{1}<\lambda_{2} .
\end{aligned}
$$

For the inverse ratio $\frac{\Phi_{2}}{\Phi_{1}}=G(R)=\frac{1}{F(R)}$, one has: 


$$
\begin{aligned}
& G(\infty)<G(R)<G(1) \text { if } \lambda_{1}>\lambda_{2}, \\
& G(1)<G(R)<G(\infty) \text { if } \lambda_{1}<\lambda_{2} .
\end{aligned}
$$

Then let

$$
L=G(1)=\frac{1}{F(1)}=1+\frac{\beta_{1}+(k+1) \beta_{2}}{\alpha_{1}+(k+1) \alpha_{2}} \text {, }
$$

and

$$
P=G(\infty)=\frac{1}{F(\infty)}=1+\frac{\beta_{2}}{\alpha_{2}}
$$

\subsubsection{Within Comparisons}

Since $v_{1}=v-n-1, v_{2}=n-1$, then:

$$
\text { (i) } \begin{aligned}
\frac{\lambda_{1}>\lambda_{2}}{1} & : \\
\mathrm{P} & <\frac{\Phi_{2}}{\Phi_{1}}<\mathrm{L}, \text { using }(8.7 .20) .
\end{aligned}
$$

$\mathrm{G}_{2}$ in $(8.5 .7)$ becomes:

$$
G_{2}=\int_{0}^{1} \frac{u^{\frac{1}{2}(v-n-3)}(1-u)^{\frac{1}{2}(n-3)}}{[u+P(1-u)]^{2}} d u \text {. }
$$

Also $G_{1}$ in $(8.5 .8)$ becomes:

$$
G_{1}=\int_{0}^{1} \frac{u^{\frac{1}{2}(v-n-3)}(1-u)^{\frac{1}{2}(n-3)}}{u+L(1-u)} d u .
$$


and

$$
\text { (ii) } \begin{aligned}
\frac{\lambda_{1}<\lambda_{2}}{} & : \\
L & <\frac{\Phi_{2}}{\Phi_{1}}<P \quad, \quad \text { using }(8.7 .20),
\end{aligned}
$$

and

$$
\begin{aligned}
& G_{2}=\int_{0}^{1} \frac{u^{\frac{1}{2}(v-n-3)}(1-u)^{\frac{1}{2}(n-3)}}{[u+L(1-u)]^{2}} d u, \\
& G_{1}=\int_{0}^{1} \frac{u^{\frac{1}{2}(v-n-3)}(1-u)^{\frac{1}{2}(n-3)}}{u+P(1-u)} d u,
\end{aligned}
$$

The above integrals can be evaluated for $n \geq 2$. The combining constant $B$ and a conservative lower bound $D$ of the recovery ratio are given in (8.5.12) and (8.5.20). These integrals have been evaluated and $B$ and $D$ has been computed for all designs listed in reference (1), except in LS6 through LS9 where the inter-model is singular. It should be noted that LS16 through LS20 are of $L_{3}$ sub-type of IS type designs, and the remaining designs are of $\mathrm{L}_{2}$ sub-type. The results are listed in Table $\mathrm{V}$.

The combined estimate, as given in (8.4.1), is:

$$
\hat{\tau}_{i}=U_{i}+\frac{\theta_{i} B^{2}}{\sum_{\substack{j=1 \\ j \neq i}}^{v-1}\left(x_{j}-U_{j}\right)^{2}}\left(x_{i}-U_{i}\right), \quad i=1,2, \ldots, n(n-I) \text { (8.7.27) }
$$


By $(8.2 .5)$,

$$
V\left(U_{i}\right)=v_{1}-C_{1}=\frac{k-c_{1}}{a} \sigma^{2} \text {, so } \theta_{i}=\frac{k-c_{1}}{a} \text {, }
$$

and then (8.7.27) becomes,

$$
\hat{\tau}_{i}=U_{i}+\frac{\left(k-c_{1}\right) B s^{2}}{a_{\substack{j=1 \\ j \neq i}}^{v-1}\left(x_{j}-U_{j}\right)^{2}}\left(x_{i}-U_{i}\right), i=1,2, \ldots, n(n-1) \text { (8.7.28) }
$$

\subsubsection{Among Comparisons}

Here $v_{1}=v-n, v_{2}=n-2$, and for:

$$
\text { (i) } \begin{aligned}
\frac{\lambda_{1}>\lambda_{2}}{L} & : \\
& \frac{1}{L}<\frac{\Phi_{1}}{\Phi_{2}}<\frac{1}{\mathrm{P}}, \quad \text { using (8.7.19). }
\end{aligned}
$$

$\mathrm{G}_{2}^{\prime}$ in $(8.5 .14)$ becomes:

$$
\begin{aligned}
G_{2}^{\prime} & =\int_{0}^{1} \frac{u^{\frac{1}{2}(v-m-2)}(1-u)^{\frac{1}{2}(n-4)}}{\left[\frac{1}{L} u+(1-u)\right]^{2}} d u, \\
& =L^{2} \int_{0}^{1} \frac{u^{\frac{1}{2}(v-n-2)}(I-u)^{\frac{1}{2}(n-4)}}{[u+L(1-u)]^{2}} d u,
\end{aligned}
$$


$G_{1}^{\prime}$ in (8.5.15) becomes:

$$
\begin{aligned}
G_{1}^{\prime} & =\int_{0}^{1} \frac{u^{\frac{1}{2}(v-n-2)}(1-u)^{\frac{1}{2}(n-4)}}{\frac{1}{P} u+(1-u)} d u, \\
& =P \int_{0}^{1} \frac{u^{\frac{1}{2}(v-n-2)}(1-u)^{\frac{1}{2}(n-4)}}{u+P(1-u)} d u .
\end{aligned}
$$

(ii) $\underline{\lambda}_{1}<\lambda_{2}$ :

$$
\begin{aligned}
& \frac{1}{P}<\frac{\Phi_{1}}{\Phi_{2}}<\frac{1}{L}, \text { using (8.7.19), hence } \\
G_{2}^{\prime}= & \int_{0}^{1} \frac{u^{\frac{1}{2}(v-n-2)}(1-u)^{\frac{1}{2}(n-4)}}{\left[\frac{1}{P} u+(1-u)\right]^{2}} d u, \\
= & P^{2} \int_{0}^{1} \frac{u^{\frac{1}{2}(v-n-2)}(1-u)^{\frac{1}{2}(n-4)}}{[u+P(1-u)]^{2}} d u,
\end{aligned}
$$

and

$$
\begin{aligned}
G_{1}^{\prime} & =\int_{0}^{1} \frac{u^{\frac{1}{2}(v-n-2)}(1-u)^{\frac{1}{2}(n-4)}}{\frac{1}{L} u+(1-u)} d u, \\
& =L \int_{0}^{1} \frac{u^{\frac{1}{2}(v-n-2)}(1-u)^{\frac{1}{2}(n-4)}}{u+L(1-u)} d u .
\end{aligned}
$$

For the convergence of the above integrals $n>2$. The combining constant $B$ and a conservative lower bound $D$ of the recovery ratio are given in $(8.5 .18)$ and $(8.5 .22)$ respectively. The above integrals along with $\mathrm{B}$ and $\mathrm{D}$ are evaluated and 
listed in Table VI for the same set of designs mentioned in Section 8.7.1.

The combined estimate is

$$
\hat{\tau}_{i}=U_{i}+\frac{\theta_{i} B s^{2}}{\sum_{\substack{j=1 \\ j \neq i}}^{v-1}\left(x_{j}-U_{j}\right)^{2}}\left(x_{i}-U_{i}\right), i=n(n-1)+1, \ldots, n^{2}-1 .
$$

By $(8.2 .5)$ and $(8.3 .1)$, one can write:

$$
v\left(U_{i}\right)=v_{1}-c_{1}+(n-i+1)\left(c_{1}-c_{2}\right)=\left[\frac{k-c_{1}}{a}+\frac{(n-i+1)\left(\lambda_{1}-\lambda_{2}\right)}{k \Delta}\right] \sigma^{2},
$$

hence:

$$
\theta_{i}=\frac{k-c_{1}}{a}+\frac{(n-1)\left(\lambda_{1}-\lambda_{2}\right)}{k \Delta} \text { for } I_{2} \text { sub-type, }
$$

and

$$
\theta_{i}=\frac{k-c_{1}}{a}+\frac{(n-2)\left(\lambda_{1}-\lambda_{2}\right)}{k \Delta} \text { for } I_{3} \text { sub-type. }
$$

Accordingly,

$$
\begin{gathered}
\hat{\tau}_{i}=U_{i}+\frac{\left[k \Delta\left(k-c_{1}\right)+(n-1) a\left(\lambda_{1}-\lambda_{2}\right)\right] B s^{2}}{a k \Delta \sum_{\substack{j=1 \\
j \neq i}}^{v-1}\left(x_{j}-U_{j}\right)^{2}}\left(x_{i}-U_{i}\right), \\
i=n(n-1)+1, \ldots, n^{2}-1,
\end{gathered}
$$

for $\mathrm{L}_{2}$ sub-type, or LSI through LS5 and LS10 through LSI5 as listed in reference (1), and 


$$
\begin{aligned}
\hat{\tau}_{i}=U_{i}+\frac{\left[k \Delta\left(k-c_{1}\right)+(n-2) a\left(\lambda_{1}-\lambda_{2}\right)\right] B S^{2}}{\left.a k \Delta \sum_{\substack{j=1 \\
j \neq i}}^{v-1}\left(x_{j}-U_{j}\right)^{2}-U_{i}\right),} \\
i=n(n-1)+1, \ldots, n^{2}-1,
\end{aligned}
$$

for $L_{3}$ sub-type, or LS16 through LS20 as listed in reference (1).

8.8 Triangular P.B.I.B. Designs

8.8.1 Definition and Comment on the Singularity of the Inter-analys is Model

In triangular designs the number of treatments can be expressed as $v=\frac{n(n-1)}{2}$, and the association scheme is an array of $\mathrm{n}$ rows and $\mathrm{n}$ columns with the following properties:

(i) The positions in the principal diagonal are left blank.

(ii) The $n(n-1) / 2$ positions above the principal diagonal are filled by the numbers $1,2, \ldots, n(n-1) / 2$ corresponding to the treatments.

(iii) The $n(n-1) / 2$ positions below the principal diagonal are filled so that the array is symmetrical about the principal diagonal. 
(iv) For any treatment $i$ the first associates are exactly those treatments which lie in the same row (or in the same column) as i.

The following relations hold

$$
\begin{aligned}
& \mathrm{n}_{1}=2 \mathrm{n}-4, \quad \mathrm{n}_{2}=(\mathrm{n}-2)(\mathrm{n}-3) / 2, \\
& \mathrm{P}_{1}=\left(\begin{array}{cc}
n-2 & \mathrm{n}-3 \\
n-3 & (\mathrm{n}-3)(\mathrm{n}-4) / 2
\end{array}\right), \\
& P_{2}=\left(\begin{array}{cc}
4 & 2 n-8 \\
2 n-8 & (n-4)(n-5) / 2
\end{array}\right) .
\end{aligned}
$$

The parameters $v, r, k, b, \lambda_{1}, \lambda_{2}$, and $n$, along with the constants $\mathrm{C}_{1}, \mathrm{C}_{2}, \Delta$, and $\mathrm{H}$, are given for 36 triangular designs in reference (I).

Reference (6) gives a set of three equations with three unknowns for each treatment resulting from Rao's combined analysis. These equations are:

$$
\begin{aligned}
& \alpha T_{j}-\beta \lambda_{1} G_{j 1}-\beta \lambda_{2} G_{j 2}=P_{j}, \\
&-\beta \lambda_{1} n_{1} T_{j}+\left(\alpha-\beta \lambda_{1} P_{11}^{1}-\beta \lambda_{2} P_{12}^{1}\right) G_{j 1} \\
&-\left(\beta \lambda_{1} P_{11}^{2}+\beta \lambda_{2} P_{12}^{2}\right) G_{j 2}=\sum_{j} P_{j 1},
\end{aligned}
$$


and

$$
\begin{aligned}
-\beta \lambda_{2}{ }_{2}{ }^{T}{ }_{j}- & \left(\beta \lambda_{1} P_{21}^{l}+\beta \lambda_{2} P_{22}^{1}\right) G_{j 1} \\
& +\left(\alpha-\beta \lambda_{1} P_{21}^{2}-\beta \lambda_{2} P_{22}^{2}\right) G_{j 2}=\sum_{j} P_{j 2},
\end{aligned}
$$

where

$$
\alpha=r\left[w \frac{k-1}{k}+\frac{w^{\prime}}{k}\right], \quad \beta=\frac{w-w^{\prime}}{k},
$$

$G_{j l}$ is the total of treatments which are first associates to the $j$-th treatment,

$\mathrm{G}_{j 2}$ is the total of treatments which are second associates to the $j$-th treatment,

$$
P_{j}=w Q_{j}+\frac{w^{\prime}}{k}\left(T_{j}-\frac{r G}{b}\right),
$$

$\sum_{j} P_{j l}$ is the sum of $P_{j}$ 's for the treatments which are first associates to the $j$-th treatment,

and

$$
\begin{aligned}
& \sum_{j} P_{j 2} \text { is the sum of } P_{j} \text { 's for the treatments which are } \\
& \text { second associates of treatment } j .
\end{aligned}
$$

From the definitions of $G_{j 1}$ and $G_{j 2}$, one can write the further relation,

$$
G_{j 1}+G_{j 2}+t_{j}=0
$$

If one lets $w=0$ in (8.8.3) through (8.8.7), the corresponding normal equations for the inter-analysis above 
are obtained:

$$
\begin{aligned}
& r \hat{t}_{j}^{\prime}+\lambda_{1} G_{1}+\lambda_{2} G_{j 2}=Q_{j}^{\prime}, \\
& n_{1} \lambda_{1} \hat{t}_{j}^{\prime}+\left(r+\lambda_{1} p_{11}^{1}+\lambda_{2} p_{12}^{1}\right) G_{j 1}+\left(\lambda_{1} p_{11}^{2}+\lambda_{2} p_{12}^{2}\right) G_{j 2}=\Sigma_{j 1}^{\prime}, \\
& n_{2} \lambda_{2} \hat{t}_{j}^{\prime}+\left(\lambda_{1} p_{21}^{1}+\lambda_{1} p_{22}^{1}\right) G_{j 1}+\left(r+\lambda_{1} p_{21}^{2}+\lambda_{2} p_{22}^{2}\right) G_{j 2}=\Sigma Q_{j 2}^{\prime},
\end{aligned}
$$

and

$$
\hat{t}_{j}^{\prime}+G_{j 1}+G_{j 2}=0
$$

Substituting the $\mathrm{p}_{j \mathrm{k}}^{i}$ 's from (8.8.2) into (8.8.9)

through (8.8.12), one obtains for the triangular designs:

$$
\begin{gathered}
r \hat{t}_{j}^{\prime}+\lambda_{1} G_{j 1}+\lambda_{2} G_{j 2}=Q_{j}^{\prime}, \\
(2 n-4) \lambda_{1} \hat{t}_{j}^{\prime}+\left[r+\lambda_{1}(n-2)+\lambda_{2}(n-3)\right] G_{j 1} \\
+\left[4 \lambda_{1}+\lambda_{2}(2 n-8)\right] G_{j 2}=\sum_{j} Q_{j 1}^{\prime}, \\
\frac{1}{2}(n-2)(n-3) \lambda_{2} \hat{t}_{j}^{\prime}+\left[\lambda_{1}(n-3)+\frac{1}{2} \lambda_{2}(n-3)(n-4)\right] G_{j 1} \\
+\left[r+\lambda_{1}(2 n-8)+\frac{1}{2} \lambda_{2}(n-4)(n-5)\right] G_{j 2}=\sum_{j} Q_{j 2}^{\prime},
\end{gathered}
$$

and

$$
\hat{t}_{j}^{\prime}+G_{j 1}+G_{j 2}=0
$$

Computing $\mathrm{G}_{\mathrm{j} 2}$ from (8.8.16) and substituting into (8.8.13) and (8.8.14), one can write:

$$
\left(r-\lambda_{2}\right) \hat{t}_{j}^{\prime}+\left(\lambda_{1}-\lambda_{2}\right) G_{j 1}=Q_{j}^{\prime},
$$


and

$$
\begin{aligned}
& \left.[2 n-4) \lambda_{1}-4 \lambda_{1}-(2 n-8) \lambda_{2}\right] \hat{t}_{j}^{\prime} \\
& +\left[r+\lambda_{1}(n-2)-4 \lambda_{1}+\lambda_{2}(n-3)-\lambda_{2}(2 n-8)\right] G_{j 1}=\sum_{j} Q_{j 1}^{\prime} .
\end{aligned}
$$

Simplifying the above equations,

$$
\left(r-\lambda_{2}\right) \hat{t}_{j}^{\prime}+\left(\lambda_{1}-\lambda_{2}\right) G_{j 1}=Q_{j}^{\prime},
$$

and

$$
(2 n-8)\left(\lambda_{1}-\lambda_{2}\right) \hat{t}_{j}^{\prime}+\left[\left(r-\lambda_{1}\right)+(n-5)\left(\lambda_{1}-\lambda_{2}\right)\right] G_{j 1}=\sum_{j} Q_{j 1}^{\prime}
$$

This set of two equations has no unique solution when the coefficients matrix is singular, i.e.,

$$
\begin{aligned}
F & =\left|\begin{array}{cc}
r-\lambda_{2} & \lambda_{1}-\lambda_{2} \\
(2 n-8)\left(\lambda_{1}-\lambda_{2}\right) & \left(r-\lambda_{1}\right)+(n-5)\left(\lambda_{1}-\lambda_{2}\right)
\end{array}\right|, \\
& =\left(r-\lambda_{1}\right)\left(r-\lambda_{2}\right)+(n-5)\left(r-\lambda_{2}\right)\left(\lambda_{1}-\lambda_{2}\right)-2(n-4)\left(\lambda_{1}-\lambda_{2}\right)^{2}=0 .
\end{aligned}
$$

\subsubsection{Application of Theorem 3 when $V\left(z_{i}\right)$ is of the Form}

\section{$\underline{V-C+\rho\left(C-C^{\prime}\right)}$}

The formula (8.4.7) in theorem 3 can be written here as:

$$
\sum_{j \neq i}^{t} z^{2}=\sum_{j \neq i}^{t}\left[v-c+\rho_{j}\left(c-c^{\prime}\right)\right] x_{I}^{2} .
$$


Suppose that $\rho=0$ for $\nu_{1} z_{i}$ 's, and $\rho>0$ for $v_{2} z_{i}$ 's, where $v_{1}+v_{2}=t-1$, then:

$$
\sum_{j \neq i}^{t} z_{j}^{2}=(v-C) x_{v_{1}}^{2}+\sum_{\substack{j=v+1 \\ j \neq i}}^{t}\left[(v-C)+p_{j}\left(C-C^{\prime}\right)\right] x_{1}^{2} \cdot(8.8 .22)
$$

In the case $\lambda_{1}>\lambda_{2}$ and in view of $(8.3 .8),(8.8 .22)$ can be written as:

$$
\begin{gathered}
\sum_{j \neq i}^{t} z_{j}^{2}<(v-c) x_{v_{1}}^{2}+\left[(v-c)+p_{\min .}\left(c-c^{\prime}\right)\right] x_{v_{2}}^{2} \\
=\psi_{1} x_{v_{1}}^{2}+\psi_{2} x_{v_{2}}^{2}=x+y_{1},
\end{gathered}
$$

and

$$
\begin{array}{r}
\sum_{j \neq i}^{t} z_{j}^{2}>(v-c) x_{v_{1}}^{2}+\left[v-c+p_{\max .}\left(c-c^{\prime}\right)\right] x_{v_{2}}^{2} \\
=\psi_{1} x_{v_{1}}^{2}+\psi_{3} x_{v_{2}}^{2}=x+y_{2},
\end{array}
$$

From (8.8.23) and (8.8.24) one can develop the formula (8.4.6) in theorem 3 as follows:

$$
\begin{aligned}
& v\left(\hat{\tau}_{i}\right)=v_{i}+\frac{v_{i}^{2} B^{2}(f+2) \Phi_{i}}{f} E \frac{1}{\left(\sum_{j \neq i}^{t} z^{2}\right)^{2}}-2 v_{i}^{2} B E \frac{1}{\sum_{j \neq i}^{t} z_{j}^{2}} \\
& \left\langle v_{i}+\frac{v_{i}^{2} B^{2}(f+2) \Phi_{i}}{f} E \frac{1}{\left(X+Y_{2}\right)^{2}}-2 v_{i}^{2} B E \frac{1}{X+Y_{1}} .\right.
\end{aligned}
$$


From (8.5.3) and (8.5.6), one can write:

$$
\begin{aligned}
& E \frac{1}{\left(X+Y_{2}\right)^{2}}=\frac{\Gamma\left(\frac{1}{2} \bar{\nu}_{1}^{+\nu} 2^{-2}\right)}{4 \Gamma\left(\frac{1}{2} \nu_{1}\right) \Gamma\left(\frac{1}{2} \nu_{2}\right) \psi_{1}^{2}} \int_{0}^{1} \frac{u^{\frac{1}{2} \nu} 1^{-1}(1-u)^{\frac{1}{2} \nu} 2^{-1}}{\left[u+\frac{\psi_{3}}{\psi_{1}}(1-u)\right]^{2}} d u \\
& <\frac{1}{(v-4)(v-6) \beta\left(\frac{1}{2} v_{1}, \frac{1}{2} v 2\right) \psi_{1}^{2}} \int_{0}^{1} \frac{u^{\frac{1}{2} v} 1^{-1}(1-u)^{\frac{1}{2} v} 2^{-1}}{[u+L(1-u)]^{2}} d u \\
& =\frac{G_{2}}{(v-4)(v-6) \beta\left(\frac{1}{2} v_{1}, \frac{1}{2} v_{2}\right) \psi_{1}^{2}},
\end{aligned}
$$

where $I$ is a lower bound of $\psi_{3} / \psi_{1}$.

Again using $(8.5 .6)$,

$$
\begin{aligned}
& E \frac{I}{X+Y}=\frac{\Gamma\left(\frac{1}{2} \nu_{1}+\nu_{2}-1\right)}{2 \Gamma\left(\frac{1}{2} \nu_{1}\right) \Gamma\left(\frac{1}{2} \nu_{2}\right) \psi_{1}} \int_{0}^{1} \frac{u^{\frac{1}{2} \nu_{1}-1}(1-u)^{\frac{1}{2} \nu} 2^{-1}}{u+\frac{\psi_{2}}{\psi_{1}}(1-u)} d u \\
& <\frac{1}{(v-3) \beta\left(\frac{1}{2} v_{1}, \frac{1}{2} v_{2}\right) \psi_{1}} \int_{0}^{1} \frac{u^{\frac{1}{2} v} 1^{-1}(1-u)^{\frac{1}{2} v} 2^{-1}}{u+P(1-u)} d u \\
& =\frac{\mathrm{G}_{1}}{(v-4) \beta\left(\frac{1}{2} \nu_{1}, \frac{1}{2} \nu_{2}\right) \psi_{1}} \text {, }
\end{aligned}
$$

where $P$ is an upper bound of $\psi_{2} / \psi_{I}$.

Substituting (8.8.26) and (8.8.27) into (8.8.25), $\mathrm{V}\left(\hat{\tau}_{i}\right)<v_{i}+\frac{\mathrm{v}_{i}^{2} B^{2}(f+2) \Phi_{i} G_{2}}{f(v-4)(v-6) \beta\left(\frac{1}{2} v_{1}, \frac{1}{2} v_{2}\right) \psi_{1}^{2}}-\frac{2 v_{i}^{2} B G_{1}}{(v-4) B\left(\frac{1}{2} v_{1}, \frac{1}{2} v_{2}\right) \psi_{1}}$. 
It is required that

$$
0<B<\frac{2 \mathrm{fG}_{1}(\mathrm{v}-6) \psi_{1}}{(\mathrm{f}+2) \mathrm{G}_{2} \Phi_{i}},
$$

and

$$
B_{\text {opt. }}=\frac{f G_{1}(v-6) \psi_{1}}{(f+2) G_{2} \Phi_{i}} \text {. }
$$

When $\Phi_{i}=\psi_{1}$, i.e., $\Phi_{i}=V-C,(8.8 .30)$ becomes:

$$
B_{\text {opt. }}=\frac{f G_{1}(v-6)}{(f+2) G_{2}} \text {. }
$$

Substituting $(8.8 .31)$ into $(8.8 .28)$,

$$
\begin{aligned}
v(\hat{\tau}) & <v_{i}+\frac{v_{i}^{2} B}{(v-4) \beta\left(\frac{1}{2} v_{1}, \frac{1}{2} v_{2}\right) \psi_{1}}\left[\frac{(f+2) \psi_{1} G_{2}}{f(v-6) \psi_{1}} \cdot \frac{f G_{1}(v-6) \psi_{1}}{(f+2) \psi_{1} G_{2}}-2 G_{1}\right], \\
& =v_{i}+\frac{v_{i}^{2}}{(v-4) \beta\left(\frac{1}{2} v_{1}, \frac{1}{2} v_{2}\right) \psi_{1}} \cdot \frac{f G_{1}(v-6)}{(f+2) G_{2}}\left(-G_{1}\right), \\
& =v_{i}-\frac{f G_{1}^{2}(v-6)}{(v-4) G_{2}(f+2) \beta\left(\frac{1}{2} v_{1}, \frac{1}{2} v_{2}\right)} \cdot \frac{v_{i}^{2}}{\psi_{1}},
\end{aligned}
$$

so that the recovery ratio is at least

$$
D=\frac{f G_{1}^{2}(v-6)}{(f+2) G_{2} \beta\left(\frac{1}{2} \nu_{1}, \frac{1}{2} \nu_{2}\right)(v-4)} .
$$

When $\Phi_{i} \neq \psi_{1}$, i.e., $v\left(z_{i}\right)=V-C+\rho\left(C-C^{\prime}\right)$ with $\rho>0$, then

$$
B_{\text {opt. }}>\frac{f G_{1}(v-6) \psi_{1}}{(f+2) \psi_{2} G_{2}}>\frac{f G_{1}(v-6)}{(f+2) G_{2} P} \text {, }
$$


where $\mathbf{P}$ is an upper bound of $\frac{\psi_{2}}{\psi_{1}}$. Thus one can take

$$
B=\frac{f G_{1}(v-6)}{(f+2) G_{2} P}
$$

In view of $(8.8 .30)$, one can write $(8.8 .28)$ as:

$$
\begin{aligned}
v\left(\hat{\tau}_{i}\right) & <v_{i}+\frac{v_{i}^{2} B}{(v-4) \beta\left(\frac{1}{2} \nu_{1}, \frac{1}{2} \nu_{2}\right) \psi_{1}}\left[\frac{(f+2) \Phi_{i} G_{2}}{f(v-6) \psi_{1}} \cdot \frac{f G_{1}(v-6) \psi_{1}}{(f+2) G_{2} \Phi_{i}}-2 G_{1}\right], \\
& <v_{i}-\frac{v_{i}^{2}}{(v-4) \beta\left(\frac{1}{2} \nu_{1}, \frac{1}{2} \nu_{2}\right) \psi_{1}} \cdot \frac{f G_{1}^{2}(v-6)}{(f+2) G_{2} P}, \\
& =v_{i}-\frac{f G_{1}^{2}(v-6)}{(v-4)(f+2) \beta\left(\frac{1}{2} \nu_{1}, \frac{1}{2} \nu_{2}\right) G_{2} P} \cdot \frac{\psi_{1}}{\psi_{1}} \cdot \frac{v_{i}^{2}}{\Phi_{i}}, \\
& <v_{i}-\frac{f G_{1}^{2}(v-6) L_{1}}{(v-4)(f+2) \beta\left(\frac{1}{2} \nu_{1}, \frac{1}{2} \nu_{2}\right) G_{2} P} \cdot \frac{v_{i}^{2}}{\Phi_{i}} .
\end{aligned}
$$

Hence the recovery ratio is at least:

$$
D=\frac{f G_{1}^{2}(v-6) L}{(v-4)(f+2) \beta\left(\frac{1}{2} \nu_{1}, \frac{1}{2} \nu_{2}\right) G_{2}^{P}}
$$

If $\lambda_{1}<\lambda_{2}$, then $\rho_{\min }$ in $(8.8 .23)$ and $\rho_{\max }$ in $(8.8 .24)$ exchange positions.

\subsubsection{Recovery of Inter-block Information in Triangular} Designs

The designs to be discussed here, are those in which the inter-analysis is not singular. Out of the 36 designs 
listed in (1), one finds, applying the formula (8.8.20), that the inter-analysis is not singular in the following designs :

T6, T7, T8, T12, T13, T14, T18, T19, in which $v=10$, and in $\mathrm{T} 23, \mathrm{~T} 24, \mathrm{~T} 30$, in which $\mathrm{v}=15$.

(a) For designs with $\mathrm{v}=10$, consider the matrix:

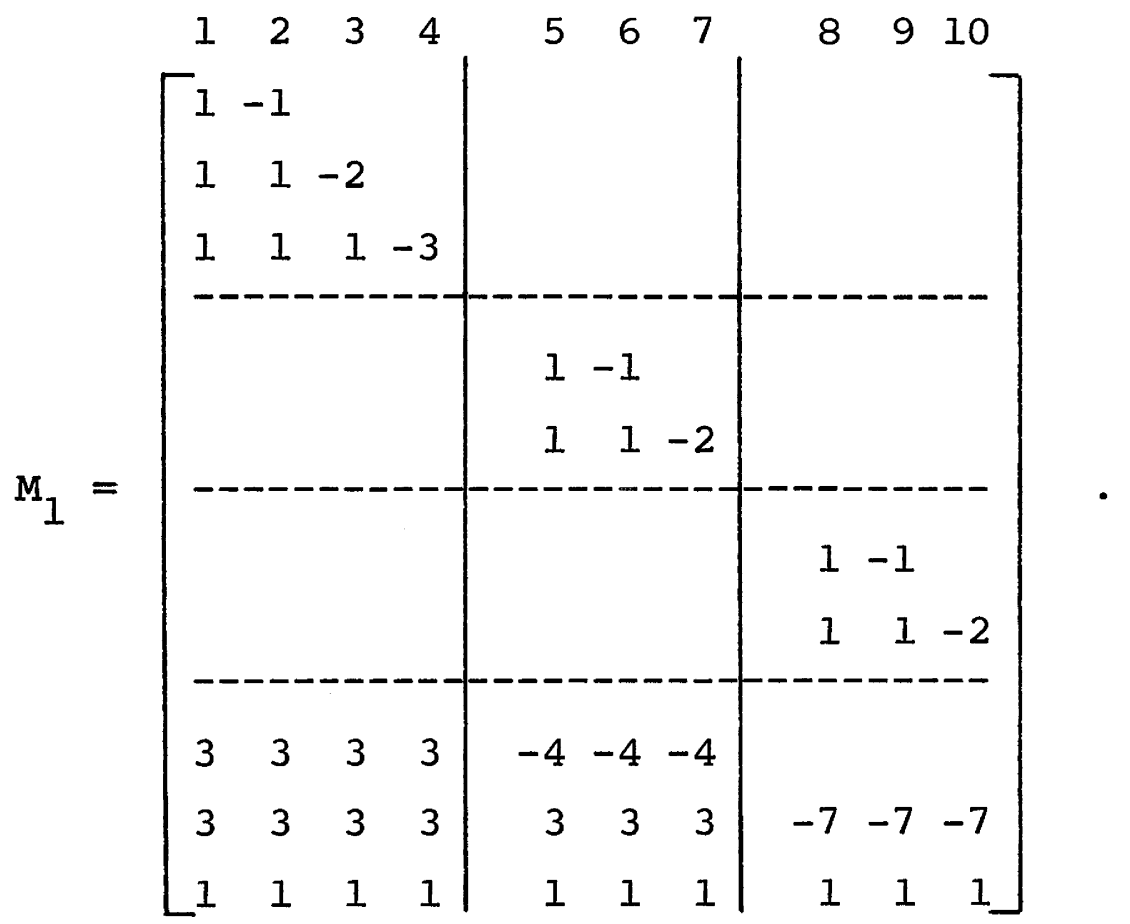
$(8.8 .38)$

Let $M$ be the orthogonal matrix obtained by normalizing the rows of $\mathrm{M}_{1}$. 
Let

$$
\underline{U}=\left[\begin{array}{l}
U_{1} \\
U_{2} \\
\vdots \\
U_{v-1} \\
0
\end{array}\right]=M\left[\begin{array}{c}
\hat{t}_{1} \\
\hat{t}_{2} \\
\vdots \\
\hat{t}_{v}
\end{array}\right]=M t,
$$

and

$$
\underline{x}=\left[\begin{array}{c}
x_{1} \\
x_{2} \\
\vdots \\
x_{v-1} \\
0
\end{array}\right]=M\left[\begin{array}{c}
\hat{t}_{1}^{\prime} \\
\hat{t}_{2}^{\prime} \\
\vdots \\
\hat{t}_{v}^{\prime} \\
v
\end{array}\right]=M \hat{t}^{\prime},
$$

where $\hat{t}_{i}, \hat{t}_{i}^{\prime}$ are the intra- and inter-estimates of the $i-t h$ treatment effect $t_{i}$, respectively. The treatments are arranged in the vector $t$ arithmetically from 1 to v. From the construction of $M_{1}$ in $(8.8 .38)$, it should be noted that the treatments have been divided into three groups; the first contains $t_{1}, t_{2}, t_{3}, t_{4}$; the second contains $t_{5}, t_{6}$, $t_{7}$ i the treatments $t_{8}, t_{9}, t_{10}$ form the third group. The first seven rows in $M_{1}$ are comparisons within groups; the next two comparisons are among groups. It is to be noticed that the treatments in the same group are mutually first 
associates. It is simple to verify that for the within comparisons :

$$
\begin{aligned}
& \mathrm{v}\left(\mathrm{U}_{i}\right)=\mathrm{v}_{1}-\mathrm{C}_{1}, \quad i=1,2, \ldots, 7, \\
& \mathrm{v}\left(\mathrm{x}_{i}\right)=\mathrm{V}_{1}^{\prime}-\mathrm{C}_{1}^{\prime}, \quad i=1,2, \ldots, 7,
\end{aligned}
$$

For among comparisons:

$$
\begin{aligned}
& \mathrm{v}\left(\mathrm{U}_{8}\right)=\mathrm{v}_{1}-\mathrm{c}_{1}+\frac{12}{7}\left(\mathrm{c}_{1}-\mathrm{c}_{2}\right), \\
& \mathrm{v}\left(\mathrm{u}_{9}\right)=\mathrm{v}_{1}-\mathrm{c}_{1}+\frac{9}{7}\left(\mathrm{c}_{1}-\mathrm{c}_{2}\right), \\
& \mathrm{v}\left(\mathrm{x}_{8}\right)=\mathrm{v}_{1}^{\prime}-\mathrm{c}_{1}^{\prime}+\frac{12}{7}\left(\mathrm{c}_{1}^{\prime}-\mathrm{c}_{2}^{\prime}\right),
\end{aligned}
$$

and

$$
\mathrm{v}\left(\mathrm{x}_{9}\right)=\mathrm{v}_{1}^{\prime}-\mathrm{C}_{1}^{\prime}+\frac{9}{7}\left(\mathrm{c}_{1}^{\prime}-\mathrm{c}_{2}^{\prime}\right)
$$

Let

$$
\underline{\mathrm{Z}}=\underline{\mathrm{X}}-\underline{\mathrm{U}},
$$

then

$$
\mathrm{V}\left(\mathrm{z}_{i}\right)=\mathrm{V}\left(\mathrm{U}_{i}\right)+\mathrm{v}\left(\mathrm{x}_{i}\right) \text {, }
$$

and using (8.8.41) through (8.8.46), one can write:

$$
\begin{aligned}
& \mathrm{v}\left(\mathrm{z}_{i}\right)=\mathrm{v}_{1}-\mathrm{C}_{1}+\mathrm{v}_{1}^{\prime}-\mathrm{C}_{1}^{\prime}=\mathrm{v}-\mathrm{C}, \quad \mathrm{i}=1,2, \ldots, 7,(8.8 .51) \\
& \mathrm{v}\left(\mathrm{z}_{8}\right)=\mathrm{v}-\mathrm{C}+\frac{12}{7}\left(\mathrm{C}-\mathrm{C}^{\prime}\right),
\end{aligned}
$$

and

$$
\mathrm{V}\left(\mathrm{z}_{9}\right)=\mathrm{V}-\mathrm{C}+\frac{9}{7}\left(\mathrm{c}-\mathrm{C}^{\prime}\right)
$$


It should be noted that $V\left(z_{i}\right)$ has been expressed in the general form $V-C+\rho\left(C-C^{\prime}\right)$, where $\rho=0$ for $z_{1}$ through $z_{7}$, and $\rho=\frac{12}{7}, \frac{9}{7}$ for $\mathbf{z}_{8}$ and $\mathbf{z}_{9}$, respectively.

(b) Similarly for designs in which $v=15$, consider the matrix:

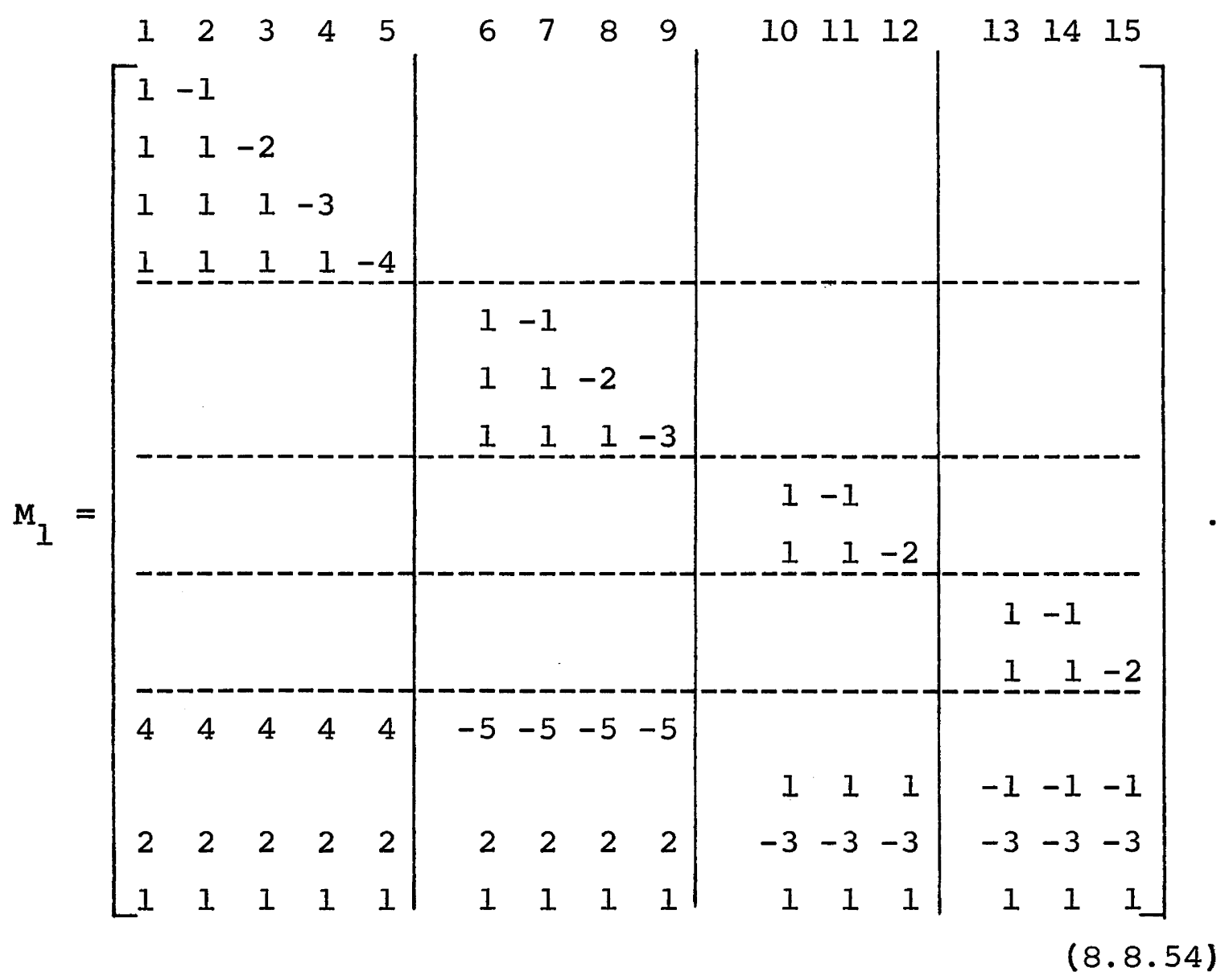

Let $M$ be the orthogonal matrix obtained from $M_{1}$ by normalizing its rows, and let $\underline{\mathrm{U}}$ and $\underline{\mathrm{X}}$ be as defined in (8.8.39) and (8.8.40), respectively. From the construction of $\mathrm{M}_{1}$ in 
(8.8.54), it should be noted that the 15 treatments have been divided into four groups. The first group contains $t_{1}$ through $t_{5}$, the second group contains $t_{6}$ through $t_{9}$, the treatments $t_{10}$ through $t_{12}$ form the third group, and the fourth group contains $t_{13}$ through $t_{15}$. The first 11 rows of $\mathrm{M}_{1}$ are comparisons within groups; the next three comparisons are among groups. The treatments within the same group are mutually first associates. For within comparisons:

$$
V\left(U_{i}\right)=v_{1}-C_{1}, \quad i=1,2, \ldots, 11,
$$

and

$$
v\left(x_{i}\right)=v_{1}^{\prime}-C_{1}^{\prime}, \quad i=1,2, \ldots, 11
$$

For among comparisons:

$$
\begin{aligned}
& \mathrm{v}\left(\mathrm{u}_{12}\right)=\mathrm{v}_{1}-\mathrm{c}_{1}+\frac{8}{3}\left(\mathrm{c}_{1}-\mathrm{c}_{2}\right), \\
& \mathrm{v}\left(\mathrm{u}_{13}\right)=\mathrm{v}_{1}-\mathrm{c}_{1}+\left(\mathrm{c}_{1}-\mathrm{c}_{2}\right), \\
& \mathrm{v}\left(\mathrm{u}_{14}\right)=\mathrm{v}_{1}-\mathrm{c}_{1}+\frac{7}{3}\left(\mathrm{c}_{1}-\mathrm{c}_{2}\right) ; \\
& \mathrm{v}\left(\mathrm{x}_{12}\right)=\mathrm{v}_{1}^{\prime}-\mathrm{c}_{1}^{\prime}+\frac{8}{3}\left(\mathrm{c}_{1}^{\prime}-\mathrm{c}_{2}^{\prime}\right), \\
& \mathrm{v}\left(\mathrm{x}_{13}\right)=\mathrm{v}_{1}^{\prime}-\mathrm{c}_{1}^{\prime}+\left(\mathrm{c}_{1}^{\prime}-\mathrm{c}_{1}^{\prime}\right),
\end{aligned}
$$

and

$$
\mathrm{v}\left(\mathrm{x}_{14}\right)=\mathrm{v}_{1}^{\prime}-\mathrm{c}_{1}^{\prime}+\frac{7}{3}\left(\mathrm{c}_{1}^{\prime}-\mathrm{c}_{2}^{\prime}\right)
$$


Taking into account $(8.8 .47)$ and $(8.8 .48)$, and using (8.8.55) through (8.8.62), one can write:

$$
\begin{aligned}
& \mathrm{v}\left(\mathrm{z}_{i}\right)=\mathrm{V}-\mathrm{C}, \quad \mathrm{i}=1,2, \ldots, 11 ; \\
& \mathrm{v}\left(\mathrm{z}_{12}\right)=\mathrm{V}-\mathrm{C}+\frac{8}{3}\left(\mathrm{C}-\mathrm{C}^{\prime}\right), \\
& \mathrm{v}\left(\mathrm{z}_{13}\right)=\mathrm{V}-\mathrm{C}+\left(\mathrm{C}-\mathrm{C}^{\prime}\right),
\end{aligned}
$$

and

$$
\mathrm{V}\left(\mathrm{z}_{14}\right)=\mathrm{V}-\mathrm{C}+\frac{7}{3}\left(\mathrm{C}-\mathrm{C}^{\prime}\right)
$$

It is to be noted also that $V\left(z_{i}\right)$ has been expressed in the general form $\mathrm{V}-\mathrm{C}+\rho\left(\mathrm{C}-\mathrm{C}^{\prime}\right)$, where $\rho=0$ for $\mathrm{z}_{1}$ through $\mathrm{z}_{11}$, and $\rho=\frac{8}{3}, 1, \frac{7}{3}$ for $\mathrm{z}_{12}, \mathrm{z}_{13}, \mathrm{z}_{14}$, respectively.

8.8.3.1 Study of the Ratio $\left[\mathrm{V}-\mathrm{C}+\rho\left(\mathrm{C}-\mathrm{C}^{\prime}\right)\right] /(\mathrm{V}-\mathrm{C})$

Now

$$
\frac{\mathrm{V}-\mathrm{C}+\rho\left(\mathrm{C}-\mathrm{C}^{\prime}\right)}{\mathrm{V}-\mathrm{C}}=1+\rho \frac{\mathrm{C}-\mathrm{C}^{\prime}}{\mathrm{V}-\mathrm{C}}
$$

By $(8.2 .16)$ and $(8.2 .17)$,

$$
C-C^{\prime}=C_{1}+C_{1}^{\prime}-C_{2}-C_{2}^{\prime}=\left(C_{1}-C_{2}\right)+\left(C_{1}^{\prime}-C_{2}^{\prime}\right)
$$

Substituting (8.2.7) and (8.3.2) into (8.8.68), one obtains:

$$
C-C^{\prime}=\frac{c_{1}-C_{2}}{a} \sigma^{2}+\frac{C_{1}^{\prime}-C_{2}^{\prime}}{r} \sigma^{\prime 2} \text {. }
$$

Substituting (8.8.69) and (8.6.16) into (8.8.67), one 
obtains:

$$
\frac{V-C+\rho\left(C-C^{\prime}\right)}{V-C}=1+\rho \frac{\frac{c_{1}-C_{2}}{a} \sigma^{2}+\frac{c_{1}^{\prime}-c_{2}^{\prime}}{r} \sigma^{\prime 2}}{\frac{k-c_{1}}{a} \sigma^{2}+\frac{k-c_{1}^{\prime}}{r} \sigma^{\prime 2}},
$$

and using (8.1.13),

$$
=1+\rho \frac{\left(\frac{c_{1}-C_{2}}{a}+\frac{c_{1}^{\prime}-c_{2}^{\prime}}{r}\right) \sigma^{2}+\frac{k\left(c_{1}^{\prime}-c_{2}^{\prime}\right)}{r} \sigma_{b}^{2}}{\left(\frac{k-c_{1}}{a}+\frac{k-c_{1}^{\prime}}{r}\right) \sigma^{2}+\frac{k\left(k-c_{1}^{\prime}\right)}{r} \sigma_{b}^{2}} \cdot(8.8 .70)
$$

Let $\frac{\sigma_{b}^{2}}{\sigma^{2}}=R>1$, then $(8.8 .70)$ becomes:

$$
\frac{V-C+\rho\left(C-C^{\prime}\right)}{V-C}=1+\rho \frac{\alpha_{1}+\beta_{1} R}{\alpha_{2}+\beta_{2} R}=F(R),
$$

where:

$$
\begin{array}{ll}
\alpha_{1}=\frac{c_{1}-c_{2}}{a}+\frac{c_{1}^{\prime}-c_{2}^{\prime}}{r}, & \beta_{1}=\frac{k\left(c_{1}^{\prime}-c_{2}^{\prime}\right)}{r}, \\
\alpha_{2}=\frac{k-c_{1}}{a}+\frac{k-c_{1}^{\prime}}{r}, & \beta_{2}=\frac{k\left(k-c_{1}^{\prime}\right)}{r},
\end{array}
$$

and $c_{1}^{\prime}, c_{2}^{\prime}$ were defined in (8.1.11).

$F(R)$ is always between the limits:

$$
F(1)=1+\rho \frac{\alpha_{1}+\beta_{1}}{\alpha_{2}+\beta_{2}} \text {, }
$$

and

$$
F(\infty)=1+\rho \frac{\beta_{1}}{\beta_{2}} .
$$




\subsubsection{Combined Estimates for Triangular Designs}

The results of Section (8.8.2) are applicable in the triangular subclass for combining the comparisons $U_{i}$ 's and $x_{i}^{\prime}$ s.

(a) For within comparisons, the combined estimate given in (8.4.1) is:

$$
\hat{\tau}_{i}=U_{i}+\frac{\theta_{i} \mathrm{Bs}^{2}}{\sum_{\substack{j=1 \\ j \neq i}}^{t}\left(x_{j}-U_{j}\right)^{2}}\left(x_{i}-U_{i}\right),
$$

where $\theta_{i}$ is the coefficient of $\sigma^{2}$ in $V\left(U_{i}\right)$. Using (8.2.5),

$$
\mathrm{V}\left(\mathrm{U}_{\mathrm{i}}\right)=\mathrm{v}_{1}-\mathrm{C}_{1}=\frac{\mathrm{k}-\mathrm{C}_{1}}{\mathrm{a}} \sigma^{2}
$$

Thus $\theta_{i}=\frac{k-c_{1}}{a}$ for every within comparison, and (8.8.75) becomes finally:

$$
\hat{\tau}_{i}=U_{i}+\frac{\left(k-c_{1}\right) B s^{2}}{a \sum_{j \neq i}^{t}\left(x_{j}-U_{j}\right)^{2}}\left(x_{i}-U_{i}\right),
$$

where $k, c_{1}, a=r k-r$, are known parameters, and $B$ is $a$ constant to be taken for a specific design from Table VII. A conservative lower bound $\mathrm{D}$ of the ratio of recovery achieved is given for each design in the same table. 
(b) For among comparisons, the variance of $U_{i}$ is of the general form:

$$
v\left(u_{i}\right)=v_{1}-C_{1}+p_{i}\left(c_{1}-C_{2}\right)
$$

By $(8.2 .5)$ and $(8.2 .7)$, one can write $(8.8 .78)$ as:

$$
V\left(U_{i}\right)=\left[\frac{k-c_{1}}{a}+\rho_{i} \frac{c_{1}-c_{2}}{a}\right] \sigma^{2}=\frac{\left(k-\rho_{i} c_{2}\right)+\left(\rho_{i}-1\right) c_{1}}{a} \sigma^{2} \text {. }
$$

Thus

$$
\theta_{i}=\frac{\left(k-\rho_{i} c_{2}\right)+\left(p_{i}-1\right) c_{1}}{a},
$$

and (8.8.75) becomes:

$$
\hat{\tau}_{i}=U_{i}+\frac{\left[\left(k-\rho_{i} c_{2}\right)+\left(\rho_{i}-1\right) c_{1}\right] B^{2}}{a \sum_{j \neq i}^{t}\left(x_{j}-U_{j}\right)^{2}}\left(x_{i}-U_{i}\right)
$$

$B$ is given for each design in Table VIII. Also in the same table, a lower bound $D$ of the recovery ratio is given. The $\rho_{i}$ 's are given in Table XI.

\subsection{Cyclic P.B.I.B. Designs with Two Associate Classes}

\subsubsection{Definition}

A non-group divisible partially balanced incomplete block design is called cyclic if the set of first associates of the i-th treatment is obtained by adding i-1 to the numbers in the set of first associates of the first treatment 
and subtracting $\mathrm{v}$, whenever the sum exceeds $\mathrm{v}$, where $\mathrm{v}$ is the number of treatments in the design. In giving the association scheme of such a design, it is, therefore, sufficient to give the first associates of the first treatment. The parameters and plans of these designs are given in reference (1). The inter-analysis in all ten designs listed is not singular.

8.9.2 Recovery of Inter-block Information in Cyclic Designs Following a similar approach to that in traingular designs, the treatments in each design will be grouped into a number of groups, with the treatments falling in one group being mutually first associates. An orthogonal matrix M will be defined in each case. The vectors $\underline{U}$ and $\underline{x}$ will be defined as:

$$
\underline{U}=M \underline{\hat{t}},
$$

and

$$
\underline{x}=M \underline{t}^{\prime} .
$$

The treatments $t_{1}, t_{2}, \ldots, t_{v}$ are arranged in the vector $\hat{t}$ or $\hat{t}^{\prime}$ according to the grouping plan; that is, the first number of treatments are those of the first group, followed by treatments of the second group, etc. A vector, 


$$
\underline{\mathbf{z}}=\underline{\mathrm{X}}-\underline{\mathrm{U}},
$$

will be considered, and the variances of $\mathbf{z}_{i}$ 's will be computed. Finally, the two linear functions $U_{i}$ and $x_{i}$ will be combined, $i=1,2, \ldots, v-1$. The $U_{v}$ and $x_{v}$ are both zero's and their combined estimate $\hat{\tau}_{\mathrm{V}}$ will be assumed to be zero also.

(a) For cyclic designs cl through $c 4$, where $\mathrm{v}=13$, consider the $13 \times 13$ matrix:

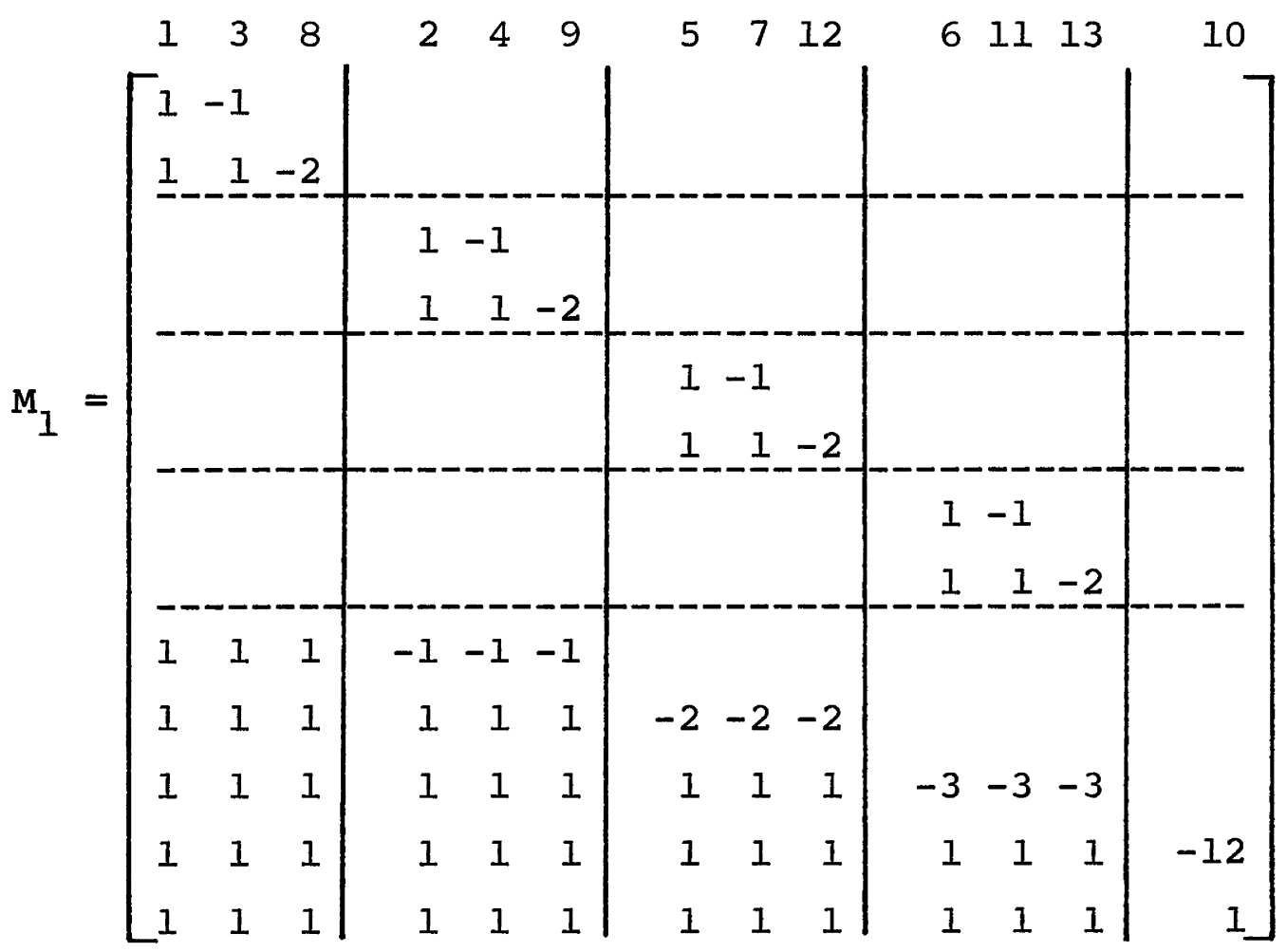

$(8.9 .4)$

The grouping plan appears on the top of the matrix, i.e., the first group includes $t_{1}, t_{3}, t_{8} ;$ the second group 
includes $t_{2}, t_{4}, t_{9}$; the third group includes $t_{5}, t_{7}, t_{12}$; the fourth group includes $t_{6}, t_{11}, t_{13} ;$ and the treatment $t_{10}$ alone forms the fifth group. Normalizing the rows of $\mathrm{M}_{1}$, the orthogonal matrices $M$ that appear in (8.9.1) and (8.9.2) are obtained. The column vectors, $\hat{t}$ in (8.9.1) and $\underline{\hat{t}}^{\prime}$ in (8.9.2), are in this case,

$$
\hat{t}=\left[\hat{t}_{1}, \hat{t}_{3}, \hat{t}_{8}, \hat{t}_{2}, \hat{t}_{4}, \hat{t}_{9}, \hat{t}_{5}, \hat{t}_{7}, \hat{t}_{12}, \hat{t}_{6}, \hat{t}_{11}, \hat{t}_{13}, \hat{t}_{10}\right],
$$

and

$$
\hat{t}^{\prime}=\left[\hat{t}_{1}^{\prime}, \hat{t}_{3}^{\prime}, \hat{t}_{8}^{\prime}, \hat{t}_{2}^{\prime}, \hat{t}_{4}^{\prime}, \hat{t}_{9}^{\prime}, \hat{t}_{5}^{\prime}, \hat{t}_{7}^{\prime}, \hat{t}_{12}^{\prime}, \hat{t}_{6}^{\prime}, \hat{t}_{11}^{\prime}, \hat{t}_{13}^{\prime}, \hat{t}_{10}^{\prime}\right] .
$$

It should be noted that the number of within comparisons is eight and that of among comparisons is four.

The variances of $\mathbf{z}_{i}$ 's are as follows:

$$
\begin{gathered}
\mathrm{v}\left(\mathrm{z}_{i}\right)=\mathrm{v}\left(\mathrm{U}_{i}\right)+\mathrm{v}\left(\mathrm{x}_{i}\right)=\mathrm{v}_{1}-\mathrm{C}_{1}+\mathrm{v}_{1}^{\prime}-\mathrm{C}_{1}^{\prime}=\mathrm{v}-\mathrm{C}, \\
i=1,2, \ldots, 8 ; \\
\mathrm{v}\left(\mathrm{z}_{9}\right)=\mathrm{v}\left(\mathrm{U}_{9}\right)+\mathrm{v}\left(\mathrm{x}_{9}\right)=\mathrm{v}-\mathrm{C}+2\left(\mathrm{C}-\mathrm{C}^{\prime}\right), \\
\mathrm{v}\left(\mathrm{z}_{10}\right)=\mathrm{v}\left(\mathrm{U}_{10}\right)+\mathrm{v}\left(\mathrm{x}_{10}\right)=\mathrm{v}-\mathrm{C}+2\left(\mathrm{C}-\mathrm{C}^{\prime}\right), \\
\mathrm{v}\left(\mathrm{z}_{11}\right)=\mathrm{v}\left(\mathrm{U}_{11}\right)+\mathrm{v}\left(\mathrm{x}_{11}\right)=\mathrm{v}-\mathrm{C}+\frac{3}{2}\left(\mathrm{C}-\mathrm{C}^{\prime}\right),
\end{gathered}
$$

and

$$
\mathrm{v}\left(\mathrm{z}_{12}\right)=\mathrm{v}\left(\mathrm{U}_{12}\right)+\mathrm{v}\left(\mathrm{x}_{12}\right)=\mathrm{v}-\mathrm{C}+\frac{1}{2}\left(\mathrm{C}-\mathrm{C}^{\prime}\right)
$$


(b) For cyclic designs c5 through c7, where $\mathrm{v}=17$, consider the $17 \times 17$ matrix:

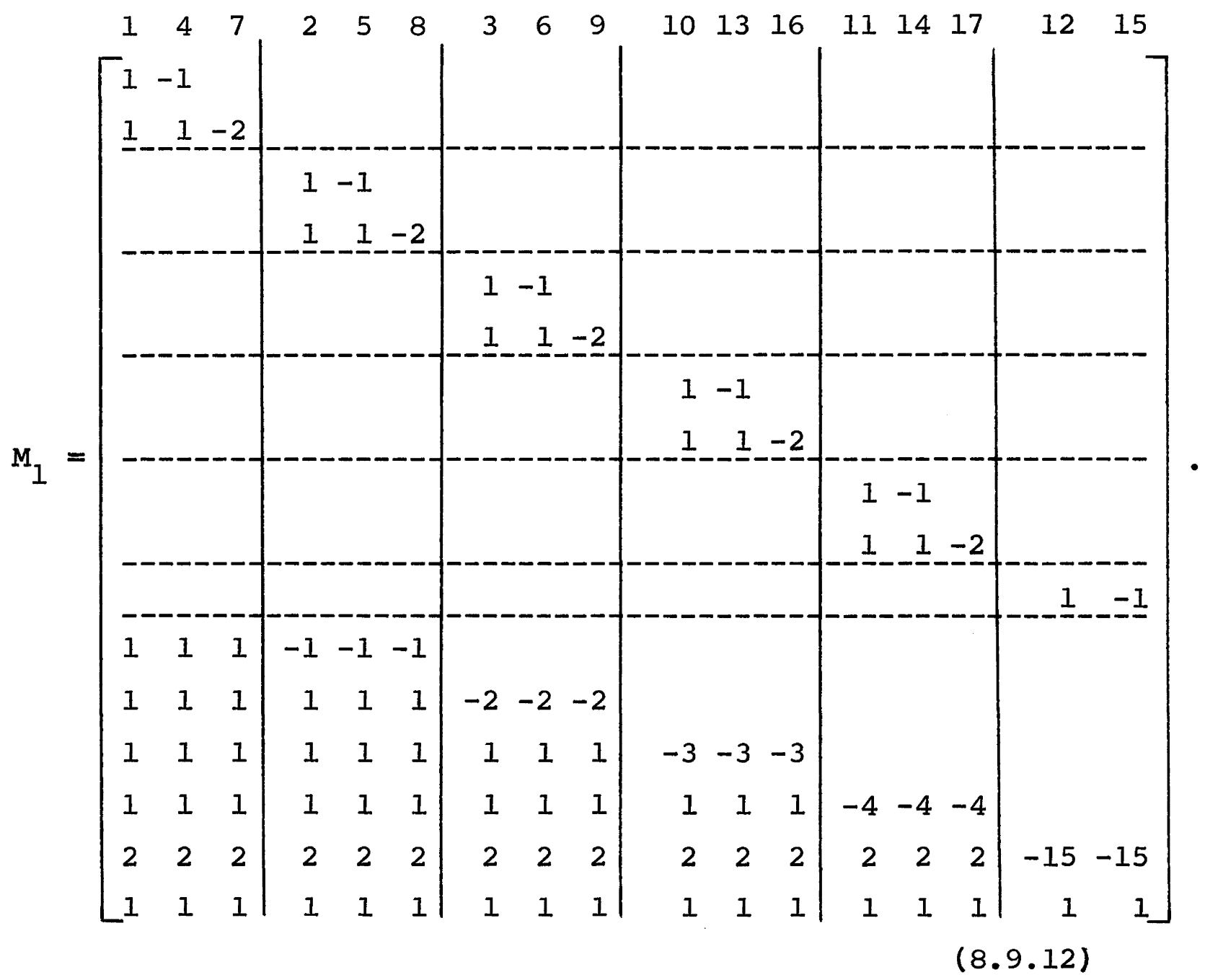

The grouping plan put in a rectangle where each row represents a group is:

$\left[\begin{array}{rrr}1 & 4 & 7 \\ 2 & 5 & 8 \\ 3 & 6 & 9 \\ 10 & 13 & 16 \\ 11 & 14 & 17 \\ 12 & 15 & \end{array}\right]$.


The variances of $\mathbf{z}_{i}$ 's are:

$$
\begin{aligned}
& \mathrm{v}\left(\mathrm{z}_{i}\right)=\mathrm{v}-\mathrm{C}, \quad, \quad i=1,2, \ldots, 11_{i} \\
& \mathrm{v}\left(\mathrm{z}_{12}\right)=\mathrm{v}-\mathrm{C}+\frac{7}{3}\left(\mathrm{c}-\mathrm{C}^{\prime}\right), \\
& \mathrm{v}\left(\mathrm{z}_{13}\right)=\mathrm{v}-\mathrm{C}+\frac{7}{3}\left(\mathrm{c}-\mathrm{C}^{\prime}\right), \\
& \mathrm{v}\left(\mathrm{z}_{14}\right)=\mathrm{v}-\mathrm{C}+\frac{5}{6}\left(\mathrm{c}-\mathrm{C}^{\prime}\right), \\
& \mathrm{v}\left(\mathrm{z}_{15}\right)=\mathrm{v}-\mathrm{C}+\frac{43}{30}\left(\mathrm{C}-\mathrm{C}^{\prime}\right),
\end{aligned}
$$

and

$$
\mathrm{V}\left(\mathrm{z}_{16}\right)=\mathrm{V}-\mathrm{C}+\frac{16}{15}\left(\mathrm{C}-\mathrm{C}^{\prime}\right)
$$

(c) For cyclic designs $c 8$ and $c 9$, where $\mathrm{v}=29$, consider the $29 \times 29$ matrix: 


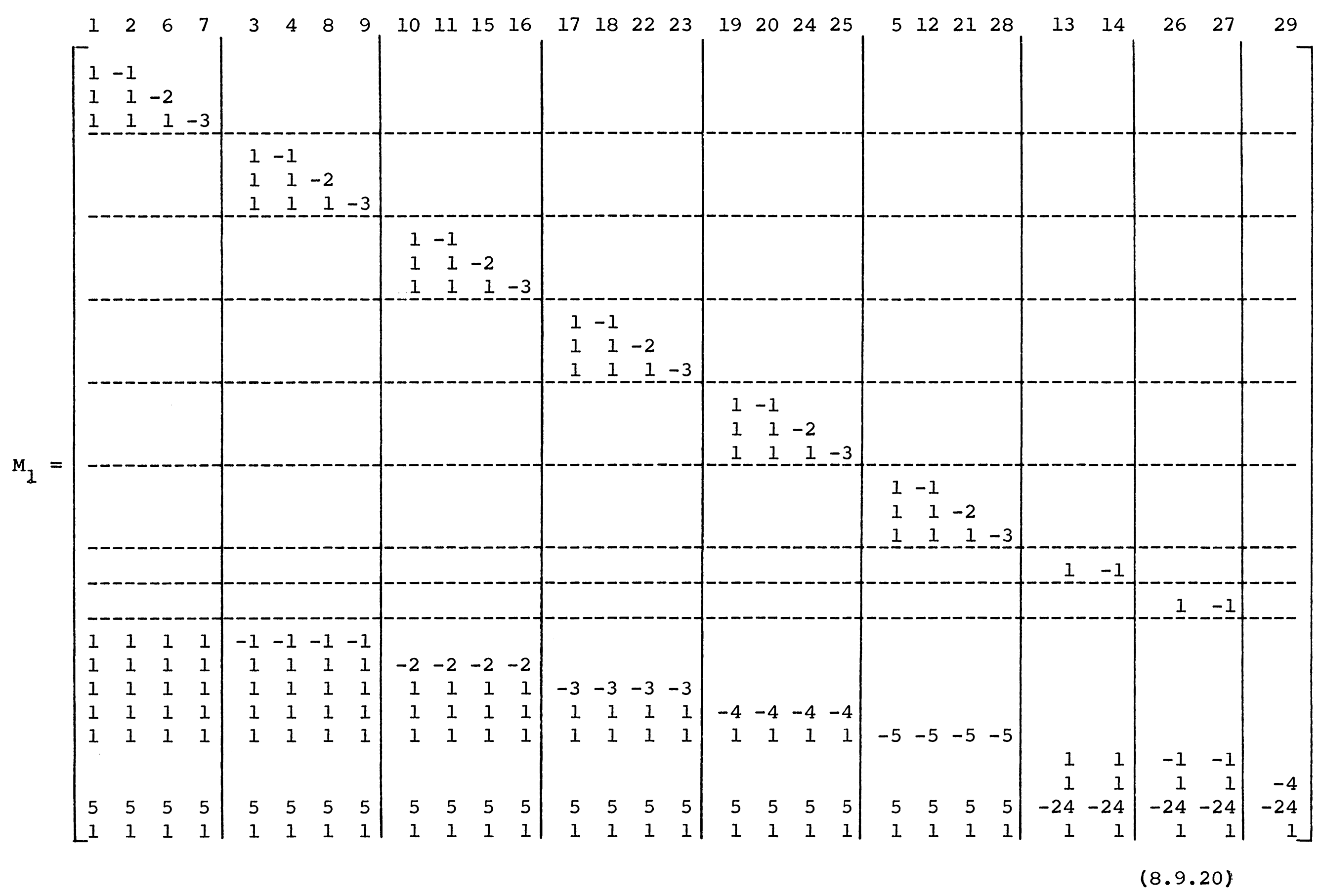

1
$\omega$
$\infty$
1 
The grouping plan put in a rectangle where each row represents a group is:

$$
\left[\begin{array}{rrrr}
1 & 2 & 6 & 7 \\
3 & 4 & 8 & 9 \\
10 & 11 & 15 & 16 \\
17 & 18 & 22 & 23 \\
19 & 20 & 24 & 25 \\
5 & 12 & 21 & 28 \\
13 & 14 & & \\
26 & 27 & & \\
29 & & &
\end{array}\right] .
$$

The variances of the $\mathbf{z}_{i}$ 's are:

$$
\begin{aligned}
& v\left(z_{i}\right)=v-C, \quad i=1,2, \ldots, 20 ; \\
& v\left(z_{21}\right)=v-C+\frac{5}{2}\left(C-C^{\prime}\right), \\
& v\left(z_{22}\right)=v-C+\frac{11}{6}\left(C-C^{\prime}\right), \\
& v\left(z_{23}\right)=v-C+\frac{61}{24}\left(C-C^{\prime}\right), \\
& v\left(z_{24}\right)=v-C+\frac{97}{40}\left(C-C^{\prime}\right), \\
& v\left(z_{25}\right)=v-C+\frac{11}{5}\left(C-C^{\prime}\right), \\
& v\left(z_{26}\right)=v-C+\left(C-C^{\prime}\right) \\
& v\left(z_{27}\right)=v-C+\left(C-C^{\prime}\right),
\end{aligned}
$$


and

$$
\mathrm{V}\left(\mathrm{z}_{28}\right)=\mathrm{V}-\mathrm{C}+\frac{1}{2}\left(\mathrm{C}-\mathrm{C}^{\prime}\right)
$$

(d) For cyclic design clo in which $\mathrm{v}=37$, consider the $37 \times 37$ matrix: 


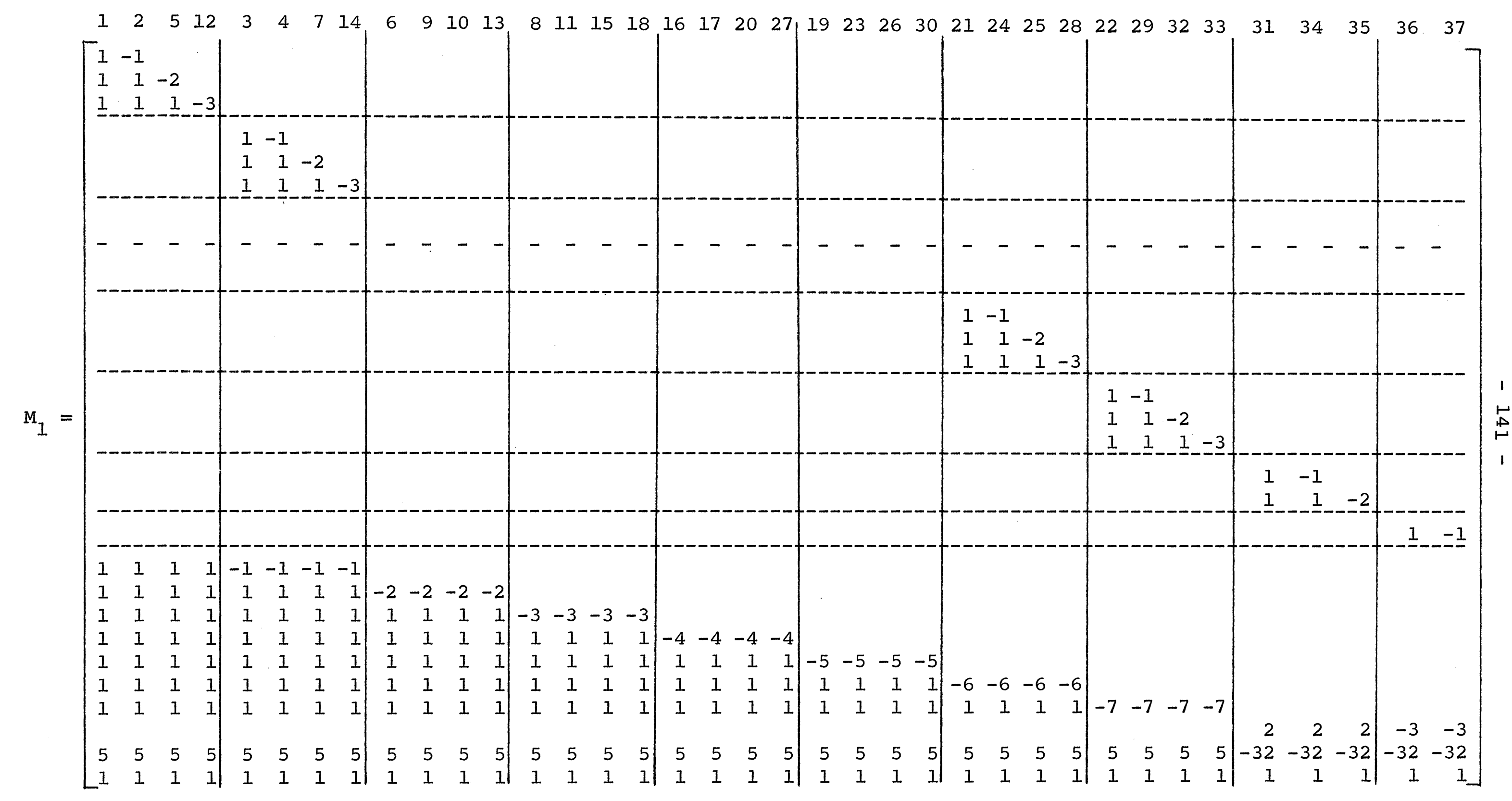

$(8.9 .31)$ 
The grouping plan put in a rectangle, where each row represents a group, is:

$$
\left[\begin{array}{rrrr}
1 & 2 & 5 & 12 \\
3 & 4 & 7 & 14 \\
6 & 9 & 10 & 13 \\
8 & 11 & 15 & 18 \\
16 & 17 & 20 & 27 \\
19 & 23 & 26 & 30 \\
21 & 24 & 25 & 28 \\
22 & 29 & 32 & 33 \\
31 & 34 & 35 & \\
36 & 37 & &
\end{array}\right]
$$

The variances of $\mathbf{z}_{i}$ 's are:

$$
\begin{aligned}
& \mathrm{v}\left(\mathrm{z}_{i}\right)=\mathrm{v}-\mathrm{C} \quad, \quad i=1,2, \ldots, 27 \\
& \mathrm{v}\left(\mathrm{z}_{28}\right)=\mathrm{v}-\mathrm{C}+\frac{5}{2}\left(\mathrm{C}-\mathrm{C}^{\prime}\right), \\
& \mathrm{v}\left(\mathrm{z}_{29}\right)=\mathrm{v}-\mathrm{C}+\frac{5}{3}\left(\mathrm{C}-\mathrm{C}^{\prime}\right), \\
& \mathrm{v}\left(\mathrm{z}_{30}\right)=\mathrm{v}-\mathrm{C}+\frac{47}{23}\left(\mathrm{C}-\mathrm{C}^{\prime}\right), \\
& \mathrm{v}\left(\mathrm{z}_{31}\right)=\mathrm{v}-\mathrm{C}+\frac{91}{40}\left(\mathrm{C}-\mathrm{C}^{\prime}\right), \\
& \mathrm{v}\left(\mathrm{z}_{32}\right)=\mathrm{v}-\mathrm{C}+\frac{11}{5}\left(\mathrm{C}-\mathrm{C}^{\prime}\right), \\
& \mathrm{v}\left(\mathrm{z}_{33}\right)=\mathrm{v}-\mathrm{C}+\frac{37}{14}\left(\mathrm{C}-\mathrm{C}^{\prime}\right), \\
& \mathrm{v}\left(\mathrm{z}_{34}\right)=\mathrm{v}-\mathrm{C}+\frac{243}{112}\left(\mathrm{C}-\mathrm{C}^{\prime}\right),
\end{aligned}
$$




$$
\mathrm{v}\left(\mathrm{z}_{35}\right)=\mathrm{v}-\mathrm{C}+\frac{8}{5}\left(\mathrm{c}-\mathrm{C}^{\prime}\right)
$$

and

$$
\mathrm{V}\left(\mathrm{z}_{36}\right)=\mathrm{V}-\mathrm{C}+\frac{3059}{2960}\left(\mathrm{C}-\mathrm{C}^{\prime}\right)
$$

\subsubsection{Combined Estimates for Cyclic Designs}

It should be noted that all the variances of $\mathbf{z}_{i}$ 's have been expressed in the general form $\mathrm{V}-\mathrm{C}+\mathrm{p}_{\mathrm{i}}\left(\mathrm{C}-\mathrm{C}^{\prime}\right)$, where $\rho_{i} \geq 0$. Hence the results of Section (8.8.2) and section (8.8.3.1) are applicable for combining $U_{i}$ 's and $x_{i}$ 's. Thus the combined estimates in section (8.8.3.2) are suitable for the cyclics under study.

(a) For within comparisons, the combined estimate is as in $(8.8 .77):$

$$
\hat{\tau}_{i}=U_{i}+\frac{\left(k-C_{1}\right) B s^{2}}{a \sum_{j \neq i}^{t}\left(x_{j}-U_{j}\right)^{2}}\left(x_{i}-U_{i}\right),
$$

where $k, c_{1}, a=r k-r$, are known parameters, and $B$ is a constant to be taken from Table IX. A conservative lower bound $D$ of the ratio of recovery achieved is given for each design in the same table. 
(b) For among comparisons, the combined estimate is, as in $(8.8 .81)$ :

$$
\hat{\tau}_{i}=U_{i}+\frac{\left[\left(k-\rho_{i} c_{2}\right)+\left(\rho_{i}-1\right) c_{1}\right] B s^{2}}{a \sum_{j \neq i}^{t}\left(x_{i}-U_{j}\right)^{2}}\left(x_{i}-U_{i}\right)
$$

$B$ is given for each design in Table $\mathrm{X}$. Also in the same table, a very conservative lower bound of the recovery ratio $D$ is given. The $\rho_{i}$ 's are given in Table XII.

\subsection{General Procedure for Recovery of Inter-block Informa-} tion in P.B.I.B. with Two Associate classes

Compute:

1. $\mathrm{Y}_{i .}=\sum_{j=1}^{b} \delta_{i j} \mathrm{y}_{i j}, \quad i=1,2, \ldots, v ;$ ( $i$-th treatment total).

2. $Y_{\cdot j}=\sum_{i=1}^{V} \delta_{i j} y_{i j}, j=1,2, \ldots, b ; \quad(j-t h$ block total)

3. $Y_{\ldots}=\sum_{i j} \delta_{i j} Y_{i j} ;$ (grand total).

4. $Q_{i}=Y_{i}$. $-\frac{1}{k}$ (sum of block totals in which treatment $i$ occurs), $i=1,2, \ldots, v ;$ (adjusted treatment totals). 
5. $S_{1}\left(Q_{i}\right), i=1,2, \ldots, v$; (the sum of $Q^{\prime} s$ for all treatments which are first associates of treatment i).

6. $\hat{t}_{i}=\frac{k-c_{2}}{a} Q_{i}+\frac{c_{1}-c_{2}}{a} s_{1}\left(Q_{i}\right), \quad i=1,2, \ldots, v ;$

(the intra-treatment estimate).

7. $Q_{i}^{\prime}=Y_{i .}-Q_{i}-\frac{Y}{V}, \quad i=1,2, \ldots, v$.

8. $S_{1}\left(Q_{i}^{\prime}\right), i=1,2, \ldots, v$.

9. $\hat{t}_{i}^{\prime}=\frac{k-c_{2}^{\prime}}{r} Q_{i}^{\prime}+\frac{c_{1}^{\prime}-c_{2}^{\prime}}{a} s_{1}\left(Q_{i}^{\prime}\right), \quad i=1,2, \ldots, v$;

(the inter-treatment estimate).

10. $\hat{t}_{i}-\hat{t}_{i}, \quad i=1,2, \ldots, v$.

11. $\underline{x}-\underline{U}=\left(\begin{array}{c}x_{1}-U_{1} \\ x_{2}-U_{2} \\ \vdots \\ x_{v-1}-U_{v-1} \\ 0\end{array}\right)=M\left(\begin{array}{c}\hat{t}_{1}^{\prime}-\hat{t}_{1} \\ \hat{t}_{2}^{\prime}-\hat{t}_{2} \\ \vdots \\ \hat{t}_{v-1}^{\prime}-\hat{t}_{v-1} \\ \hat{t}_{v}-\hat{t}_{v}\end{array}\right)=M\left(\underline{t}^{\prime}-\underline{t}\right) ;$

where $M$ is the orthogonal matrix defined in each case previous ly

12. $s^{2}=\frac{1}{f}\left(\sum_{i j} \delta_{i j} Y_{i j}^{2}-S S T-\frac{1}{k} \sum_{j=1}^{b} Y^{2} \cdot j\right) ;$ (error mean square, $f=b k-b-v+l)$. 
To combine $\mathrm{x}_{i}$ and $\mathrm{U}_{i}$, compute:

13. $\sum_{j=1}^{V}\left(x_{j}-U_{j}\right)^{2}$.

14. $\sum_{j \neq i}^{V}\left(x_{j}-U_{j}\right)^{2}=\sum_{j=1}^{V}\left(x_{j}-U_{j}\right)^{2}-\left(x_{i}-U_{i}\right)^{2}$.

15. $J=\frac{\theta_{i} \text { Bs }^{2}}{\sum_{j \neq i}\left(x_{j}-U_{j}\right)^{2}}$, where $\theta_{i}$ was defined previously

in each case; $B$ is a constant to be taken from the corresponding table.

16. The combined estimate of $\mathrm{U}_{i}$ and $\mathrm{x}_{i}$,

$$
\hat{\tau}_{i}=U_{i}+J\left(x_{i}-U_{i}\right), \quad i=1,2, \ldots, v \text {. }
$$

If the combined estimates of the treatments $t_{i}$ 's themselves are desired, one should compute:

$$
\underline{T}=\left(\begin{array}{c}
T_{1} \\
T_{2} \\
\vdots \\
T_{v}
\end{array}\right)=M^{\prime}\left(\begin{array}{c}
\hat{\tau}_{1} \\
\hat{\tau}_{2} \\
\vdots \\
\hat{\tau}_{v}
\end{array}\right)=M^{\prime} \hat{\tau}^{\prime},
$$

where $M^{\prime}$ is the transpose of $M$. 


\subsection{Comment on the Numerical Methods Used in Table VII}

\section{Through Table $\mathrm{x}$}

In Section 7.1.1, a method of utilizing the Hypergeometric series and Gauss' continued fraction was discussed. In Table VII through $\mathrm{x}$, the same idea was applied for computing $\mathrm{E}_{1}$ and $\mathrm{E}_{2}$, where:

$$
E_{1}=\frac{1}{\beta\left(\frac{1}{2} \nu_{1}, \frac{1}{2} \nu_{2}\right)} \int_{0}^{1} \frac{u^{\frac{1}{2} \nu_{1}}{ }^{-1}(1-u)^{\frac{1}{2} \nu} 2^{-1}}{u+P(1-u)} d u, \quad \text {, (8.11.1) }
$$

and

$$
\mathbf{E}_{2}=\frac{1}{\beta\left(\frac{1}{2} v_{1}, \frac{1}{2} v_{2}\right)} \int_{0}^{1} \frac{u^{\frac{1}{2} v} 1(1-u)^{\frac{1}{2} v} 2^{-1}}{[u+L(1-u)]^{2}} d u
$$

In view of $(7.1 .8),(7.1 .9)$, and $(7.1 .11),(8.11 .1)$ may be written as:

$$
\begin{aligned}
E_{1} & =\frac{1}{P \beta\left(\frac{1}{2} v_{1}, \frac{1}{2} v_{2}\right)} \int_{0}^{1} \frac{u^{\frac{1}{2} v_{1}-1}(1-u)^{\frac{1}{2} \nu} 2^{-1}}{1-\frac{P-1}{P} u} d u, \\
& =\frac{F\left(\frac{1}{2} v_{1}, 1, \frac{v-2}{2}, \frac{P-1}{P}\right)}{P} .
\end{aligned}
$$

Now,

$$
F\left(\frac{1}{2} v_{1}, 1, \frac{v-2}{2}, \frac{P-1}{P}\right) \text { could be put in the form of }
$$

(7.1.12) and evaluated. 
Similarly, for $\mathrm{E}_{2}$, one can write:

$$
\begin{aligned}
E_{2} & =\frac{1}{L^{2} \beta\left(\frac{1}{2} v_{1}, \frac{1}{2} v_{2}\right)} \int_{0}^{1} \frac{u^{\frac{1}{2} v_{1}-1}(1-u)^{\frac{1}{2} \nu} 2^{-1}}{\left[1-\frac{L-1}{I} u\right]^{2}} d u, \\
& =\frac{F\left(2, \frac{1}{2} v_{1}, \frac{v-2}{2}, \frac{L-1}{L}\right)}{L^{2}}
\end{aligned}
$$

To evaluate $F\left(2, \frac{1}{2} v_{1}, \frac{V-2}{2}, \frac{L-1}{L}\right)$, the following recursion formula (see reference 13, page 363) is needed:

$F(a, b, c ; z)=F(a, b+1, c+1 ; z)-\frac{a(c-b) z}{c(c+1)} F(a+1, b+1, c+2 ; z)$,

or

$F(a+1, b+1, c+2 ; z)=\frac{c(c+1)}{a(c-b) z}[F(a, b+1, c+1 ; z)-F(a, b, c ; z)] ;$

putting $\mathrm{a}=1$, one gets:

$F(2, b+1, c+2 ; z)=\frac{c(c+1)}{(c-b) z}[F(1, b+1, c+1 ; z)-F(1, b, c ; z)] .(8.11 .7)$

Comparing $(8.11 .4)$ and $(8.11 .7)$, one obtains:

$$
\begin{array}{ll}
\mathrm{b}+1=\frac{\mathrm{l}}{2} \mathrm{v}_{1}, & \mathrm{~b}=\frac{\mathrm{l}}{2} v_{1}-1, \\
\mathrm{c}+2=\frac{\mathrm{v}-2}{2}, & \mathrm{c}=\frac{\mathrm{v}-6}{2}
\end{array}
$$

and

$$
z=\frac{L-1}{L}
$$


Thus, substituting in (8.11.7),

$$
\begin{aligned}
F\left(2, \frac{1}{2} \nu_{1}, \frac{V-2}{2} ; \frac{L-1}{L}\right)= & \frac{\frac{v-6}{2} \cdot \frac{V-4}{2}}{\left(\frac{v-6}{2}-\frac{1}{2} v_{1}+1\right) \frac{L-1}{L}}\left[F\left(1, \frac{1}{2} v_{1}, \frac{V-4}{2} ; \frac{L-1}{L}\right)\right. \\
& \left.\quad-F\left(1, \frac{1}{2} v_{1}-1, \frac{V-6}{2} ; \frac{L-1}{L}\right)\right] .(8.11 .9)
\end{aligned}
$$

In view of $(7.1 .11)$ and the fact that $v_{1}+v_{2}=v-2$, (8.11.9) may be written as:

$$
\begin{aligned}
F\left(\frac{1}{2} \nu 2,2, \frac{V-2}{2} ; \frac{L-1}{L}\right)= & \frac{(v-6)(v-4) \cdot L}{4\left(\frac{1}{2} \nu_{2}-1\right)(L-1)}\left[F\left(\frac{1}{2} \nu, 1, \frac{V-4}{2} ; \frac{L-1}{L}\right)\right. \\
& \left.\quad-F\left(\frac{1}{2} \nu_{1}-1,1, \frac{V-6}{2} ; \frac{L-1}{L}\right)\right] .(8.11 .10)
\end{aligned}
$$

Now, $F\left(\frac{1}{2} \nu_{1}, 1, \frac{V-4}{2} ; \frac{\mathrm{L}-1}{\mathrm{~L}}\right)$ and $F\left(\frac{1}{2} \nu_{I}-1,1, \frac{\mathrm{V}-6}{2} ; \frac{\mathrm{L}-1}{\mathrm{~L}}\right)$ can be put in the form of Gauss' continued fraction as in (7.1.12) and evaluated.

The above was programmed for an IBM 1620 and used for cyclic designs (Tables IX and $\mathrm{X}$ ); however, as we notice from (8.11.10), the above method is not applicable when $\frac{1}{2} v 2^{-1}=0$ or $v_{2}=2$, the case that was encountered in triangular designs (Tables VII and VIII). Whenever this happened the integrals, 


$$
G_{1}=\int_{0}^{1} \frac{u^{\frac{1}{2} \nu} 1(1-u)^{\frac{1}{2} \nu} 2^{-1}}{u+P(1-u)} d u
$$

and

$$
\mathrm{G}_{2}=\int_{0}^{1} \frac{u^{\frac{1}{2} \nu} 1^{-1}(1-u)^{\frac{1}{2} \nu} 2^{-1}}{[u+L(1-u)]^{2}} d u,
$$

were evaluated by the simpson rule. The accuracy of the Simpson rule was listed in the tables; the accuracy of $\mathbf{E}_{1}$ or $\mathbb{E}_{2}$ was set to the sixth decimal place constantly. 


\section{SUMMARY AND DISCUSSION}

In summary, this dissertation has brought up the following points :

1. Under certain conditions, a new method of combining two independent estimates has been given in theorem 1 . This new method has its immediate application in incomplete block designs, in similar experiments, and in randomized block designs with heterogeneous variances. The amount of improvement obtained by this new method is very satisfactory, compared with the utmost possible theoretical improvement.

2. A procedure for recovering the inter-block information in balanced incomplete block designs was given which is applicable in experiments as small as $t=4$.

3. A generalization of theorem 1 was given which shows that the idea of recovering the inter-block information can be practically utilized in any incomplete block design with seven treatments or more.

4. For a partially balanced incomplete block design with two associate classes, a separate development was given to each of its four subclasses, namely the group divisible (G.D.), Latin square type $\left(L_{2} S\right.$ type and $I_{3} s$ type), the 
triangular, and the cyclic designs. A combined estimate was given for each case, and a general procedure for recovery of inter-block information in these subclasses was developed. Because of the special nature of singular and semi-regular G.D. designs, an inter-analysis was discussed for G.D.'s in general and for these two cases in special, and a partial utilization of the inter-information was made possible. The essence of this work arises from the fact that so far a method for recovering the inter-block information was based on the common sense of the consistency property of an estimate. Consequently, the applicability of the resulting method would make sense only in big size experiments. The resulting combined estimates are of so complicated expression that a mathematical study of their merits or demerits is not feasible, even in their domain of applicability. On the contrary, this dissertation provides a general method by a mathematical treatment. This work can be said to have the following two merits:

1. It makes possible the utilization of the inter-block information in small size experiments, as small as four treatments in B.I.B. designs and as small as seven treatments in P.B.I.B. designs. This is of special importance because of 
the fact that the intra-block efficiency is in general lower in small and moderate size designs. It follows that the recovery is needed more in this sort of designs.

2. As a ratio of the utmost possible theoretical recovery, either exactly or a lower bound of the amount of the ratio of recovery is always computable; this ratio was tabled for all B.I.B. designs listed in reference (2), and P.B.I.B. designs listed in reference (1). The ratio of recovery depends on the structure of the design; it always increases with $\mathrm{v}$, the number of treatments.

A glance at the tables, where these ratios are listed, shows that the new methods, for B.I.B. and P.B.I.B. designs, give good results where the old method is not applicable; where the old method starts, hopefully, to be valid, the ratio of recovery achieved by the new methods begins to approach the theoretical value that can be achieved, assuming the intra- and inter-variance are known 


\section{ACKNOWLEDGEMENTS}

I wish to express my sincere thanks to Professor C. Y. Kramer who introduced me to the subject and guided me during the course of this work; and to Professor B. Harshbarger for his encouragement. Thanks are due also to Mrs. Lela Barnhisel for her extreme care in typing this dissertation. 


\section{TABLE OF REFERENCES}

1. Bose, R.C., w. H. Clatworthy, and S.S. Shrikhande, (1954). North Carolina Agricultural Experiment Station, Technical Bulletin No. 107.

2. Cochran and Cox, (1957). Experimental Design. New York, John wiley and Sons.

3. Graybill and Weeks, (1959). "Combined inter- and intrablock information in balanced incomplete blocks." A.M.S. 30, 799 .

4. and Deal, (1959). "Combined unbiased estimators." Biometrics, $15,543$.

5. Kramer, C.Y., (1957). "Examples of intra-block analysis for factorials in group divisible, partially balanced incomplete block designs." Biometrics, 13.

6. Kempthorne, 0., (1952). Design of Experiments. New York, John wiley and Sons.

7. Rao, C.R., (1947). "General method of analysis for incomplete block designs." J.A.S.A., 42, 541.

8. Seshadri, V., (1961). "Estimation in the balanced incomplete block designs." Unpublished Ph.D. thesis, Oklahoma State University.

9. Biometrics, 19, 163.

10. Yates, (1937). "The recovery of inter-block information in balanced incomplete block designs." Annals of Eugenics, 10, 317.

11. Zelen, M. (1957). "The analysis of incomplete block designs." J.A.S.A., 52, 204. 
12. Bailey, w.N., (1935). Generalized Hypergeometric Series. Cambridge Tracts in Mathematics and Mathematical Physics, No. 32, 4.

13. Wall, H.S., (1948). Analytic Theory of Continued Fractions. D. Van Nostrand Company, Inc. 


\section{The vita has been removed from the scanned document}


- $158-$

T A B I E S 
Table I: $\frac{1}{\sigma^{2}}$ [percentage gain due to the Rao Method]

No. of

$0 \frac{1}{10(1+k)} \frac{1}{5(1+k)} \frac{3}{10(1+k)} \frac{4}{10(1+k)} \frac{5}{10(1+k)} \frac{6}{10(1+k)} \frac{7}{10(1+k)} \frac{8}{10(1+k)} \frac{9}{10(1+k)} \frac{1}{1+k}$ Design

$\begin{array}{llllllllllll}\text { R1 } & 0 & .000 & .001 & .002 & .003 & .004 & .005 & .006 & .008 & .010 & .012 \\ \text { R2 } & 0 & .000 & .001 & .001 & .002 & .002 & .003 & .004 & .005 & .006 & .007 \\ \text { R3 } & 0 & .000 & .001 & .001 & .002 & .003 & .004 & .005 & .006 & .007 & .009 \\ \text { R4 } & 0 & .000 & .000 & .001 & .001 & .002 & .003 & .003 & .004 & .005 & .007 \\ \text { R5 } & 0 & .000 & .001 & .002 & .003 & .003 & .004 & .006 & .007 & .008 & .009 \\ \text { R6 } & 0 & .000 & .001 & .002 & .003 & .003 & .004 & .006 & .007 & .008 & .009 \\ \text { R7 } & 0 & .000 & .001 & .001 & .002 & .002 & .003 & .004 & .005 & .006 & .007 \\ \text { R8 } & 0 & .001 & .001 & .002 & .002 & .003 & .004 & .005 & .006 & .007 & .008 \\ \text { R9 } & 0 & .000 & .001 & .001 & .002 & .002 & .003 & .004 & .004 & .005 & .006 \\ \text { R10 } & 0 & .001 & .001 & .002 & .003 & .004 & .005 & .005 & .006 & .007 & .008 \\ \text { R11 } & 0 & .000 & .000 & .001 & .001 & .002 & .003 & .004 & .004 & .005 & .006 \\ \text { R12 } & 0 & .000 & .000 & .001 & .001 & .002 & .002 & .003 & .004 & .004 & .005 \\ \text { R13 } & 0 & .000 & .001 & .001 & .002 & .003 & .004 & .004 & .005 & .007 & .008 \\ \text { R14 } & 0 & .000 & .001 & .001 & .001 & .002 & .002 & .003 & .004 & .004 & .005 \\ \text { R15 } & 0 & .000 & .000 & .000 & .001 & .001 & .001 & .002 & .002 & .003 & .003 \\ \text { R16 } & 0 & .000 & .000 & .001 & .001 & .001 & .002 & .002 & .003 & .004 & .004 \\ \text { R17 } & 0 & .000 & .000 & .001 & .001 & .001 & .002 & .002 & .003 & .003 & .004 \\ \text { R18 } & 0 & .000 & .000 & .001 & .001 & .001 & .002 & .002 & .003 & .003 & .004 \\ \text { R19 } & 0 & .000 & .000 & .001 & .001 & .001 & .002 & .002 & .002 & .003 & .003 \\ \text { R20 } & 0 & .000 & .001 & .001 & .002 & .002 & .003 & .004 & .004 & .005 & .006 \\ \text { R21 } & 0 & .000 & .000 & .001 & .001 & .001 & .002 & .002 & .003 & .004 & .004 \\ \text { R22 } & 0 & .000 & .000 & .001 & .001 & .001 & .002 & .002 & .003 & .003 & .004 \\ \text { R23 } & 0 & .000 & .000 & .000 & .001 & .001 & .001 & .002 & .002 & .003 & .003 \\ \text { R24 } & 0 & .000 & .000 & .000 & .001 & .001 & .001 & .002 & .002 & .002 & .003 \\ \text { R25 } & 0 & .000 & .000 & .000 & .001 & .001 & .001 & .002 & .002 & .003 & .003\end{array}$


Table I: (continued)

$\mathbf{u}$
$0 \frac{1}{10(1+k)} \frac{1}{5(1+k)} \frac{3}{10(1+k)}$
$\frac{4}{10(1+k)} \frac{5}{10(1+k)}$
$\frac{6}{10(1+x)} \frac{7}{10(1+x)}$
$\frac{8}{10(1+x)}$
$\frac{9}{10(1+k)}$
$\frac{1}{1+k}$

Ho. of

Dasign

\begin{tabular}{|c|c|c|c|c|c|c|c|c|c|c|c|}
\hline R26 & 0 & .000 & .000 & .000 & .001 & .001 & .001 & .002 & .002 & .002 & .003 \\
\hline R27 & 0 & .000 & .000 & .001 & .001 & .001 & .002 & .002 & .003 & .003 & .004 \\
\hline R28 & 0 & .000 & .000 & .001 & .001 & .001 & .002 & .002 & .003 & .003 & .004 \\
\hline R29 & 0 & .000 & .000 & .000 & .001 & .001 & .001 & .002 & .002 & .003 & .003 \\
\hline 230 & 0 & .000 & .000 & .001 & .001 & .001 & .002 & .002 & .003 & .003 & .004 \\
\hline R31 & 0 & .000 & .000 & .000 & .001 & .001 & .001 & .002 & .002 & .003 & .003 \\
\hline $\mathbf{2 3 2}$ & 0 & .000 & .000 & .000 & .001 & .001 & .001 & .002 & .002 & .002 & .003 \\
\hline R33 & 0 & .000 & .000 & .001 & .001 & .001 & .002 & .002 & .003 & .003 & .004 \\
\hline 234 & 0 & .000 & .000 & .001 & .001 & .001 & .001 & .002 & .002 & .002 & .003 \\
\hline R35 & 0 & .000 & .000 & .001 & .001 & .002 & .002 & .003 & .003 & .004 & .004 \\
\hline R36 & 0 & .000 & .000 & .000 & .001 & .001 & .001 & .001 & .002 & .002 & .002 \\
\hline $\mathbf{R 3 7}$ & 0 & .000 & .000 & .001 & .001 & .001 & .001 & .002 & .002 & .003 & .003 \\
\hline $\mathbf{2 3 8}$ & 0 & .000 & .000 & .000 & .001 & .001 & .001 & .002 & .002 & .002 & .003 \\
\hline $\mathbf{R} 39$ & 0 & .000 & .000 & .000 & .000 & .001 & .001 & .001 & .002 & .002 & .002 \\
\hline 840 & 0 & .000 & .000 & .001 & .001 & .001 & .002 & .002 & .003 & .003 & .004 \\
\hline 241 & 0 & .000 & .000 & .000 & .000 & .001 & .001 & .001 & .002 & .002 & .002 \\
\hline 242 & 0 & .000 & .000 & .000 & .000 & .001 & .001 & .001 & .001 & .002 & .002 \\
\hline 243 & 0 & .000 & .000 & .000 & .001 & .001 & .001 & .001 & .002 & .002 & .002 \\
\hline 244 & 0 & .000 & .000 & .000 & .000 & .001 & .001 & .001 & .002 & .002 & .002 \\
\hline 245 & 0 & .000 & .000 & .000 & .000 & .001 & .001 & .001 & .001 & .001 & .002 \\
\hline 846 & 0 & .000 & .000 & .000 & .000 & .000 & .001 & .001 & .001 & .001 & .002 \\
\hline R47 & 0 & .000 & .000 & .000 & .001 & .001 & .001 & .002 & .002 & .002 & .003 \\
\hline $\mathbf{R 4 8}$ & 0 & .000 & .000 & .000 & .000 & .001 & .001 & .001 & .001 & .002 & .002 \\
\hline R49 & 0 & .000 & .000 & .000 & .000 & .000 & .001 & .001 & .001 & .001 & .001 \\
\hline 250 & 0 & .000 & .000 & .000 & .000 & .001 & .001 & .001 & .001 & .001 & .002 \\
\hline
\end{tabular}


Table I: (continued)

$\mathrm{u} \quad 0 \frac{1}{10(1+\mathrm{k})} \frac{1}{5(1+\mathrm{k})} \frac{3}{10(1+\mathrm{k})} \frac{4}{10(1+\mathrm{k})} \frac{5}{10(1+\mathrm{k})} \frac{6}{10(1+\mathrm{k})} \frac{7}{10(1+\mathrm{k})} \frac{8}{10(1+\mathrm{k})} \frac{9}{10(1+\mathrm{k})} \frac{1}{1+\mathrm{k}}$ No. of

Design

\begin{tabular}{|c|c|c|c|c|c|c|c|c|c|c|c|c|}
\hline R51 & 0 & .000 & .000 & .000 & .000 & .000 & .000 & .001 & .001 & .001 & .001 & \\
\hline R52 & 0 & .000 & .000 & .000 & .000 & .000 & .001 & .001 & .001 & .001 & .001 & \\
\hline R53 & 0 & .000 & .000 & .000 & .000 & .000 & .001 & .001 & .001 & .001 & .001 & \\
\hline R54 & 0 & .000 & .000 & .000 & .000 & .000 & .001 & .001 & .001 & .001 & .001 & \\
\hline R55 & 0 & .000 & .000 & .000 & .000 & .000 & .001 & .001 & .001 & .001 & .001 & \\
\hline R56 & 0 & .000 & .000 & .000 & .000 & .000 & .000 & .001 & .001 & .001 & .001 & \\
\hline R57 & 0 & .000 & .000 & .000 & .000 & .000 & .000 & .001 & .001 & .001 & .001 & \\
\hline R58 & 0 & .000 & .000 & .000 & .000 & .000 & .000 & .000 & .000 & .000 & .000 & 1 \\
\hline R59 & 0 & .000 & .000 & .000 & .000 & .000 & .000 & .000 & .000 & .001 & .001 & $\mapsto$ \\
\hline R60 & 0 & .000 & .000 & .000 & .000 & .000 & .000 & .000 & .000 & .001 & .001 & ? \\
\hline R6I & 0 & .000 & .000 & .000 & .000 & .000 & .000 & .000 & .000 & .001 & .001 & I \\
\hline $\mathrm{R} 62$ & 0 & .000 & .000 & .000 & .000 & .000 & .000 & .000 & .000 & .001 & .001 & \\
\hline R63 & 0 & .000 & .000 & .000 & .000 & .000 & .000 & .000 & .000 & .000 & .000 & \\
\hline R64 & 0 & .000 & .000 & .000 & .000 & .000 & .000 & .000 & .000 & .000 & .000 & \\
\hline R65 & 0 & .000 & .000 & .000 & .000 & .000 & .000 & .000 & .000 & .000 & .000 & \\
\hline R66 & 0 & .000 & .000 & .000 & .000 & .000 & .000 & .000 & .000 & .000 & .000 & \\
\hline $\mathrm{R} 67$ & 0 & .000 & .000 & .000 & .000 & .000 & .000 & .000 & .000 & .000 & .000 & \\
\hline R68 & 0 & .000 & .000 & .000 & .000 & .000 & .000 & .000 & .000 & .000 & .000 & \\
\hline
\end{tabular}


Table II: Balanced Designs

\begin{tabular}{|c|c|c|c|c|c|c|c|c|c|c|c|c|}
\hline $\mathbf{s}$ & $\mathrm{v}$ & $r$ & $\mathrm{k}$ & $\mathrm{b}$ & $\lambda$ & $\mathrm{f}$ & $\mathbf{E}$ & $\mathrm{F}^{*}$ & B & $\mathrm{D}_{2}$ & $D_{3}$ & $D_{1}$ \\
\hline 1 & 4 & 3 & 2 & 6 & 1 & 3 & .67 & .38017290 & .526 & .18 & .20 & 0.00 \\
\hline 2 & 4 & 3 & 3 & 4 & 2 & 5 & .89 & $"$ & .626 & .20 & .24 & 0.00 \\
\hline 3 & 5 & 4 & 2 & 10 & 1 & 6 & .62 & .27639332 & 1.357 & .34 & .38 & .23 \\
\hline 4 & 5 & 6 & 3 & 10 & 3 & 16 & .83 & " & 1.608 & .40 & .44 & .28 \\
\hline 5 & 5 & 4 & 4 & 5 & 3 & 11 & .94 & $"$ & 1.531 & .38 & .42 & .26 \\
\hline 6 & 6 & 5 & 2 & 15 & 1 & 10 & .60 & .21540524 & 2.321 & .46 & .50 & .40 \\
\hline 7 & 6 & 5 & 3 & 10 & 2 & 15 & .80 & " & 2.458 & .49 & .53 & .42 \\
\hline 8 & 6 & 10 & 3 & 20 & 4 & 35 & .80 & $"$ & 2.635 & .53 & .57 & .45 \\
\hline 9 & 6 & 10 & 4 & 15 & 6 & 40 & .90 & $"$ & 2.653 & .53 & .57 & .46 \\
\hline 10 & 6 & 5 & 5 & 6 & 4 & 19 & .96 & $"$ & 2.520 & .50 & .54 & .43 \\
\hline 11 & 7 & 6 & 2 & 21 & 1 & 15 & .58 & .17614569 & 3.339 & .56 & .59 & .51 \\
\hline 12 & 7 & 3 & 3 & 7 & 1 & 8 & .78 & $"$ & 3.028 & .50 & .53 & .47 \\
\hline 13 & 7 & 4 & 4 & 7 & 2 & 15 & .88 & $"$ & 3.339 & .56 & .59 & .51 \\
\hline 14 & 7 & 6 & 6 & 7 & 5 & 29 & .97 & $"$ & 3.541 & .59 & .62 & .55 \\
\hline 15 & 8 & 7 & 2 & 28 & 1 & 21 & .57 & .14899933 & 4.377 & .63 & .65 & .60 \\
\hline 16 & 8 & 7 & 4 & 14 & 3 & 35 & .86 & $"$ & 4.535 & .65 & .68 & .61 \\
\hline 17 & 8 & 7 & 7 & 8 & 6 & 41 & .98 & $"$ & 4.571 & .65 & .68 & .62 \\
\hline 18 & 9 & 8 & 2 & 36 & 1 & 28 & .56 & .12916656 & 5.419 & .68 & .70 & .66 \\
\hline 19 & 9 & 8 & 4 & 18 & 3 & 46 & .84 & " & 5.565 & .70 & .72 & .63 \\
\hline 20 & 9 & 10 & 5 & 18 & 5 & 64 & .90 & $"$ & 5.631 & .70 & .73 & .68 \\
\hline 21 & 9 & 8 & 6 & 12 & 5 & 52 & .94 & $"$ & 5.591 & .70 & .72 & .68 \\
\hline 22 & 9 & 8 & 8 & 9 & 7 & 55 & .98 & $"$ & 5.603 & .70 & .72 & .68 \\
\hline 23 & 10 & 9 & 2 & 45 & 1 & 36 & .56 & .11405141 & 6.461 & .72 & .74 & .70 \\
\hline 24 & 10 & 9 & 3 & 30 & 2 & 51 & .74 & $"$ & 6.562 & .73 & .75 & .71 \\
\hline 25 & 10 & 6 & 4 & 15 & 2 & 36 & .83 & $"$ & 6.461 & .72 & .74 & .70 \\
\hline 26 & 10 & 9 & 5 & 18 & 4 & 63 & .89 & $"$ & 6.610 & .73 & .75 & .72 \\
\hline 27 & 10 & 9 & 6 & 15 & 5 & 66 & .93 & $"$ & 6.619 & .74 & .75 & .72 \\
\hline 28 & 10 & 9 & 9 & 10 & 8 & 71 & .99 & $"$ & 6.633 & .74 & .76 & .72 \\
\hline
\end{tabular}


Table II: (continued)

\begin{tabular}{|c|c|c|c|c|c|c|c|c|c|c|c|c|}
\hline $\mathrm{s}$ & $\mathrm{v}$ & $r$ & $\mathrm{k}$ & b & $\lambda$ & $\mathrm{f}$ & $\mathrm{E}$ & F* & B & $\mathrm{D}_{2}$ & $D_{3}$ & $\mathrm{D}_{1}$ \\
\hline 29 & 11 & 10 & 2 & 55 & 1 & 45 & .55 & .10214480 & 7.499 & .75 & .77 & .74 \\
\hline 30 & 11 & 5 & 5 & 11 & 2 & 34 & .88 & $"$ & 7.397 & .74 & .76 & .73 \\
\hline 31 & 11 & 6 & 6 & 11 & 3 & 45 & .92 & " & 7.499 & .75 & .72 & .74 \\
\hline 32 & 11 & 10 & 10 & 11 & 9 & 89 & .99 & $"$ & 7.660 & .77 & .78 & .75 \\
\hline 33 & 13 & 6 & 3 & 26 & 1 & 40 & .72 & .08457048 & 9.384 & .78 & .79 & .77 \\
\hline 34 & 13 & 4 & 4 & 13 & 1 & 27 & .81 & $"$ & 9.174 & .76 & .78 & .76 \\
\hline 35 & 13 & 9 & 9 & 13 & 6 & 92 & .96 & $"$ & 9.644 & .80 & .82 & .80 \\
\hline 36 & 15 & 7 & 3 & 35 & 1 & 56 & .71 & .07220328 & 11.462 & .82 & .84 & .81 \\
\hline 37 & 15 & 7 & 7 & 15 & 3 & 76 & .92 & " & 11.567 & .83 & .84 & .82 \\
\hline 38 & 15 & 8 & 8 & 15 & 4 & 91 & .94 & $"$ & 11.616 & .83 & .84 & .82 \\
\hline 39 & 16 & 6 & 6 & 16 & 2 & 65 & .89 & .06729477 & 12.494 & .83 & .84 & .83 \\
\hline 40 & 16 & 9 & 6 & 24 & 3 & 105 & .89 & $"$ & 12.638 & .84 & .85 & .84 \\
\hline 41 & 16 & 10 & 10 & 16 & 6 & 129 & .96 & " & 12.682 & .85 & .85 & .84 \\
\hline 42 & 19 & 9 & 3 & 57 & 1 & 97 & .70 & .05591668 & 15.576 & .87 & .87 & .86 \\
\hline 43 & 19 & 9 & 9 & 19 & 4 & 134 & .94 & .05591668 & 15.663 & .87 & .88 & .87 \\
\hline 44 & 19 & 10 & 10 & 19 & 5 & 153 & .95 & " & 15.692 & .87 & .88 & .87 \\
\hline 45 & 21 & 10 & 3 & 70 & 1 & 120 & .70 & .05026207 & 17.613 & .88 & .89 & .88 \\
\hline 46 & 21 & 5 & 5 & 21 & 1 & 64 & .84 & " & 17.364 & .87 & .87 & .87 \\
\hline 47 & 21 & 10 & 7 & 30 & 3 & 160 & .90 & " & 17.685 & .88 & .89 & .88 \\
\hline 48 & 25 & 8 & 4 & 50 & 1 & 126 & .78 & .04181724 & 21.578 & .90 & .90 & .90 \\
\hline 49 & 25 & 9 & 9 & 25 & 3 & 176 & .93 & $"$ & 21.674 & .90 & .91 & .90 \\
\hline 50 & 28 & 9 & 4 & 63 & 1 & 162 & .78 & .03714312 & 24.625 & .91 & .91 & .91 \\
\hline 51 & 28 & 9 & 7 & 36 & 2 & 189 & .89 & " & 24.668 & .91 & .92 & .91 \\
\hline 52 & 31 & 6 & 6 & 31 & 1 & 125 & .86 & .03341029 & 27.496 & .92 & .92 & .92 \\
\hline 53 & 31 & 10 & 10 & 31 & 3 & 249 & .93 & $"$ & 27.713 & .92 & .93 & .92 \\
\hline 54 & 37 & 9 & 9 & 37 & 2 & 260 & .91 & .02782200 & 33.687 & .94 & .94 & .93 \\
\hline 55 & 41 & 10 & 5 & 82 & 1 & 288 & .82 & .02503212 & 37.690 & .94 & .94 & .94 \\
\hline 56 & 57 & 8 & 8 & 57 & 1 & 343 & .89 & .01786875 & 53.652 & .96 & .96 & .96 \\
\hline 57 & 73 & 9 & 9 & 73 & 1 & 512 & .90 & .01389509 & 69.697 & .97 & .97 & .97 \\
\hline 58 & 91 & 10 & 10 & 91 & 1 & 729 & .91 & .01111421 & 87.735 & .97 & .98 & .97 \\
\hline
\end{tabular}


Table* III: Regular G.D., Within Comparisons

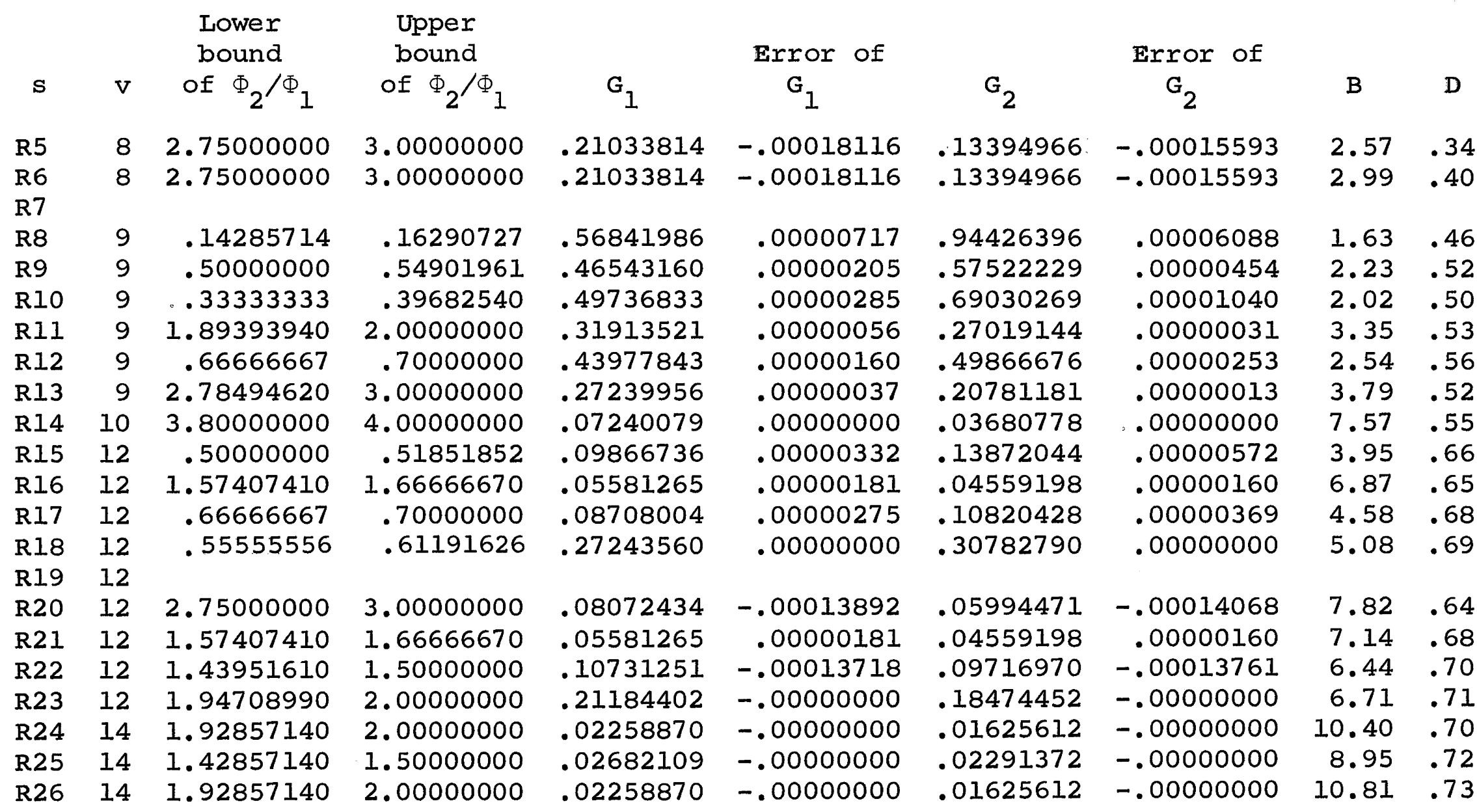

$*_{I}$ and $G_{2}$ are computed by the simpson rule in Table III through Table VI. 
Table III: (continued)

\begin{tabular}{|c|c|c|c|c|c|c|c|c|c|}
\hline$s$ & $\mathrm{v}$ & $\begin{array}{r}\text { Lower } \\
\text { bound } \\
\text { of } \Phi_{2} / \Phi_{1}\end{array}$ & $\begin{array}{r}\text { Upper } \\
\text { bound } \\
\text { of } \Phi_{2} / \Phi_{1}\end{array}$ & $\mathrm{G}_{1}$ & $\begin{array}{l}\text { Error of } \\
G_{1}\end{array}$ & $G_{2}$ & $\begin{array}{l}\text { Error of } \\
\mathrm{G}_{2}\end{array}$ & B & D \\
\hline 27 & 15 & 3.80000000 & 00000000 & .02249261 & .00000000 & .01406606 & .00000002 & 13.52 & \\
\hline R28 & 15 & 6670 & 2.00000000 & .03139546 & .00000000 & .02615760 & .00000000 & 10.35 & \\
\hline R29 & 15 & . 66666667 & . 70769231 & .04453214 & .00000000 & .05079515 & .00000000 & 7.66 & \\
\hline R30 & 15 & 3.80000000 & 4.00000000 & .02249261 & .00000000 & .01406606 & .00000002 & 14.02 & \\
\hline R31 & 15 & 3.35858590 & 3.50000000 & .13773795 & .00000002 & .11094994 & -.0000 & 10.89 & \\
\hline R32 & 15 & .58333333 & 7816 & .19287289 & .000 & .20999550 & .000 & 8.05 & \\
\hline R33 & 15 & .50000000 & .56000000 & .04709829 & .00000000 & .05832701 & .00000000 & 7.08 & \\
\hline R34 & 15 & 1.57948720 & 1.600 & .0 & .000 & .0 & 000 & 10.22 & \\
\hline R35 & 16 & 2.75000000 & 3.00000000 & .0467 & .0001 & $.03^{\prime}$ & 304 & 12.06 & \\
\hline R36 & 16 & .50000000 & .52884615 & .07203230 & -.00013834 & .08256627 & -.00013773 & 8.43 & \\
\hline R37 & 16 & .33333333 & 6774 & & -.000 & & -.000 & 7.98 & \\
\hline 38 & 16 & . 63636364 & .685 & .0 & -.00 & .0 & -.0 & 8.82 & \\
\hline R39 & 16 & 1.93918920 & 2.00 & .05368 & -.000 & .0 & -.000 & 11.38 & \\
\hline R40 & 18 & 1.28 & 1.33 & & & & 000 & 3.04 & \\
\hline 41 & 18 & 7778 & 770 & & .0 & & & 0.24 & \\
\hline R42 & 20 & 1. 24285710 & 1. 28571430 & .00294074 & .000 & .00 & .000 & 15.08 & \\
\hline R43 & 20 & .37500000 & . 41826923 & .04575421 & -.00014053 & .0523 & -.00013975 & 12.05 & $\bullet$ \\
\hline 44 & 21 & 57140 & 1.50 & & -.0 & & -.00 & 16.31 & \\
\hline 245 & 24 & 4.83 & 000 & & & & 27 & 8.73 & \\
\hline R46 & 24 & 1.69531250 & 0000 & 99 & -.0000 & .00 & -.000 & 1.04 & \\
\hline R47 & 24 & 2.75000000 & 3.00000000 & .02330 & -.00014602 & .019 & -.00014788 & 20.74 & נס. \\
\hline 48 & 24 & 1.66666670 & 410 & & & & & 19.57 & \\
\hline R49 & 24 & & & & .00000002 & & -.00000001 & 16.82 & \\
\hline R50 & 24 & 4.83333330 & 5.00000000 & .00286382 & .00000123 & .00174614 & .00000127 & 29.18 & 81 \\
\hline
\end{tabular}


Table III: (continued)

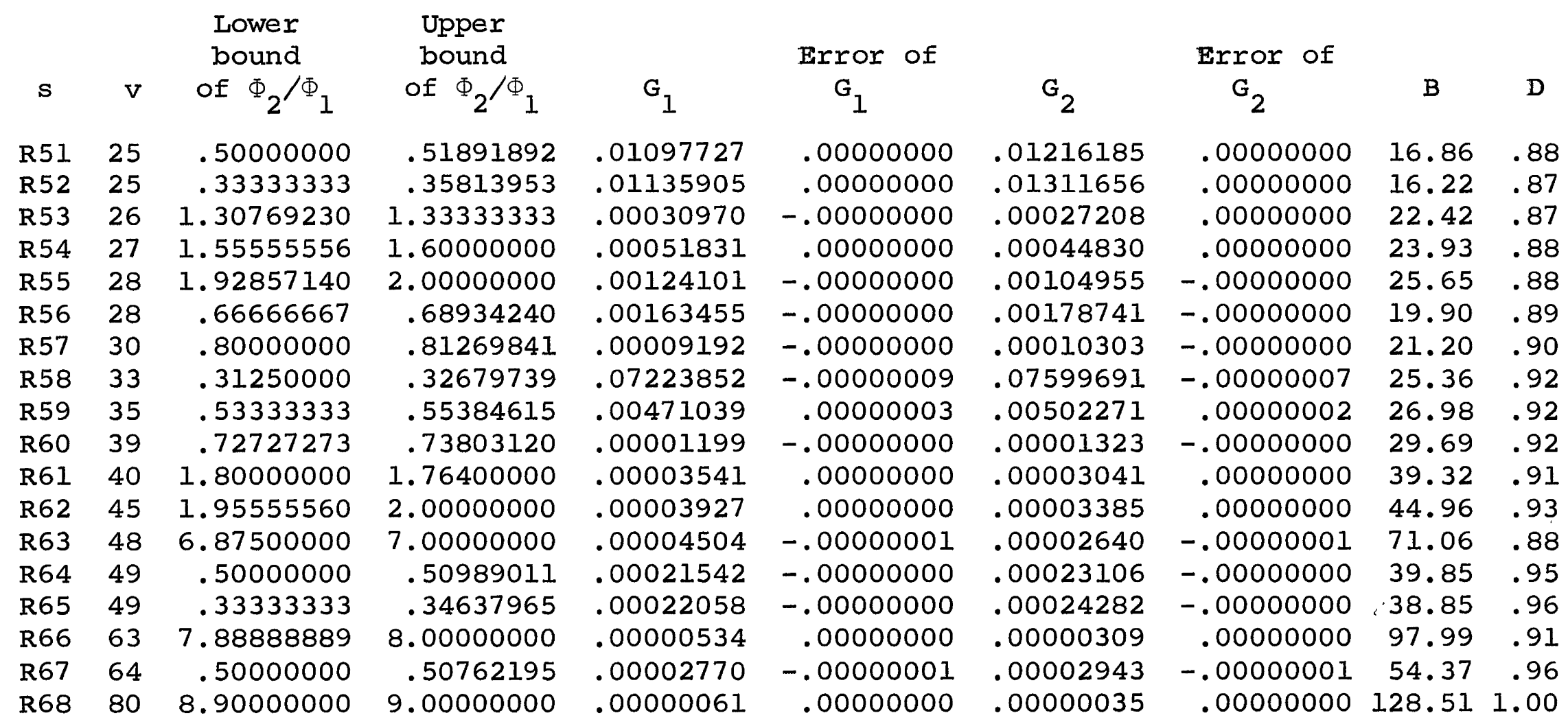


Table** IV: Regular G.D., Among Comparisons

\begin{tabular}{|c|c|c|c|c|c|c|c|c|c|}
\hline $\mathbf{s}$ & $\mathrm{v}$ & $G_{1}$ & Error of $G_{1}$ & $\mathrm{G}_{2}$ & Error of $G_{2}$ & B & D & $\frac{1}{2} \nu_{1}$ & $\frac{1}{2} v_{2}$ \\
\hline R5 & 8 & .92660598 & -.00000000 & 2.02812200 & -.00000001 & .75 & .35 & 2 & 1 \\
\hline R6 & 8 & .92660598 & -.00000000 & 2.02812200 & -.00000001 & .87 & .40 & 2 & 7 \\
\hline \multicolumn{10}{|l|}{ R7 } \\
\hline R8* & 9 & .18084145 & & .04166931 & & 11.78 & .40 & 3 & \\
\hline R9* & 9 & .58054100 & & . 37645660 & & 4.24 & .46 & 3 & 8 \\
\hline R10* & 9 & .40114170 & & .21142836 & & 5.31 & .40 & $"$ & \\
\hline RI1* & 9 & 1.82060710 & & 3.43970230 & & 1.50 & .51 & $"$ & \\
\hline RI2* & 9 & .75044440 & & .57646628 & & 3.74 & .53 & $"$ & \\
\hline RI3* & 9 & 2.47599780 & & 6.70399280 & & 1.07 & .50 & " & $"$ \\
\hline R14 & 10 & .39800645 & -.00013841 & .92952501 & -.00014245 & 1.65 & .56 & $5 / 2$ & \\
\hline R15 & 12 & .05296100 & -.00000000 & .03599987 & -.00000000 & 8.17 & .65 & 3 & 2 \\
\hline R16 & 12 & .10760015 & .00000000 & .14907694 & .00000000 & 4.05 & .65 & $"$ & \\
\hline RI7 & 12 & .06449589 & -.00000000 & .05349805 & -.00000000 & 6.86 & .66 & $"$ & \\
\hline R18* & 12 & .50211678 & & .35168153 & & 8.20 & .60 & $9 / 2$ & \\
\hline \multicolumn{10}{|l|}{ R19 } \\
\hline R20 & 12 & .52964673 & -.00000000 & 1.31482570 & -.00000000 & 2.34 & .62 & 4 & \\
\hline R21 & 12 & .10760015 & .00000000 & .14907694 & .00000000 & 4.21 & .68 & 3 & 2 \\
\hline R22 & 12 & .33216065 & -.00000000 & .47197854 & -.00000000 & 4.10 & .68 & 4 & 1 \\
\hline R23* & 12 & 1.54557910 & & 2.93234110 & & 3.08 & .70 & $9 / 2$ & \\
\hline R24 & 14 & .05199792 & .00000114 & .07729396 & .00000116 & 5.03 & .71 & $7 / 2$ & \\
\hline R25 & 14 & .04482904 & .00000114 & .05774582 & .00000115 & 5.93 & .72 & $"$ & \\
\hline R26 & 14 & .05199792 & .00000114 & .07729396 & .00000116 & 5.23 & .74 & $"$ & \\
\hline
\end{tabular}

*Starred experiments have $G_{2}$ and $G_{1}$ as improper integrals; these integrals were evaluated to the fourth decimal place.

** Lower and upper bounds of $\Phi_{2} / \Phi_{1}$ are given in Table III. 
Table IV: (continued)

\begin{tabular}{|c|c|c|c|c|c|c|c|c|c|}
\hline $\mathbf{s}$ & $\mathrm{V}$ & $\mathrm{G}_{1}$ & Error of $G_{1}$ & $\mathrm{G}_{2}$ & Error of $\mathrm{G}_{2}$ & B & $\mathrm{D}$ & $\frac{1}{2} v_{1}$ & $\frac{1}{2} v_{2}$ \\
\hline R27 & 15 & .18129859 & -.00014162 & .50217754 & -.00014551 & 3.05 & .68 & 5 & $3 / 2$ \\
\hline R28 & 15 & .11611093 & -.00013932 & .20327436 & -.00014065 & 4.93 & .70 & . & $"$ \\
\hline R29 & 15 & .05347859 & -.00013793 & .04282467 & -.00013764 & 10.91 & .72 & " & \\
\hline R30 & 15 & .18129859 & -.00014162 & .50217754 & -.00014551 & 3.17 & .71 & " & " \\
\hline R31* & 15 & 2.16696860 & & 6.96629570 & & 2.73 & .73 & 6 & $1 / 2$ \\
\hline $\mathrm{R} 32$ * & 15 & .44699438 & & .32561219 & & 12.04 & .66 & $"$ & \\
\hline R33 & 15 & .04206644 & -.00013774 & .02922470 & -.00013730 & 12.62 & .65 & 5 & $3 / 2$ \\
\hline R34 & 15 & .10340764 & -.00013898 & . 14842798 & -.00013971 & 6.15 & .78 & " & \\
\hline R35 & 16 & .37627084 & -.00000001 & .99309690 & -.00000000 & 3.64 & .68 & 6 & 1 \\
\hline R36 & 16 & .09018610 & -.00000000 & .05430287 & .00000000 & 16.04 & .72 & $"$ & 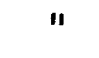 \\
\hline R37 & 16 & .06200081 & -.00000000 & .02843526 & .00000000 & 21.19 & .66 & $"$ & \\
\hline R38 & 16 & .11214239 & -.00000000 & .08637660 & -.00000000 & 12.67 & .71 & $"$ & \\
\hline R39 & 16 & .28767554 & -.00000000 & .52623532 & -.00000001 & 5.35 & .77 & $"$ & \\
\hline $\mathrm{R} 40$ & 18 & .00877483 & -.00000000 & .01045750 & -.00000000 & 9.82 & .80 & $9 / 2$ & $7 / 2$ \\
\hline R41 & 18 & .00661897 & -.00000000 & .00595953 & -.00000000 & 13.04 & .80 & $"$ & 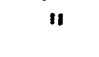 \\
\hline $\mathrm{R} 42$ & 20 & .00401108 & .00000000 & .00467043 & .00000000 & 11.79 & .83 & 5 & 4 \\
\hline $\mathrm{R} 43$ & 20 & .05062569 & & .02532839 & -.00000000 & 27.58 & .70 & 8 & 1 \\
\hline $\mathrm{R} 44$ & 21 & .01032799 & .00000118 & .01424629 & .00000118 & 10.67 & .81 & 7 & $5 / 2$ \\
\hline $\mathrm{R} 45$ & 24 & .03353262 & .00000002 & .11118008 & .00000005 & 5.28 & .80 & 9 & 2 \\
\hline $\mathrm{R} 46$ & 24 & .00398032 & -.00000000 & .00597248 & -.00000000 & 11.77 & .84 & 8 & 3 \\
\hline R47 & 24 & .24051216 & -.00000006 & .67638281 & .00000012 & 6.30 & .76 & 10 & 1 \\
\hline $\mathrm{R} 48$ & 24 & .01588761 & .00000001 & .02485663 & .00000001 & 11.34 & .81 & 9 & 2 \\
\hline R49* & 24 & . 28425893 & & . 16907593 & & 29.88 & .77 & $21 / 2$ & $1 / 2$ \\
\hline R50 & 24 & .03353262 & .00000002 & .11118008 & .00000005 & 5.37 & .81 & 9 & 2 \\
\hline
\end{tabular}


Table IV: (continued)

\begin{tabular}{|c|c|c|c|c|c|c|c|c|c|}
\hline $\mathbf{s}$ & $\mathrm{V}$ & $\mathrm{G}_{1}$ & Error of $G_{1}$ & $\mathrm{G}_{2}$ & Error of $\mathrm{G}_{2}$ & B & $\mathrm{D}$ & $\frac{1}{2} v_{1}$ & $\frac{1}{2} v_{2}$ \\
\hline R52 & 25 & .00989200 & -.00014338 & .00418149 & -.00014265 & 44.30 & .77 & & \\
\hline R54 & 27 & .00132261 & -.00000001 & .00185870 & -.00000001 & 14.73 & .86 & 9 & $7 / 2$ \\
\hline R55 & 28 & .00518073 & .00000122 & .00904618 & .00000123 & 12.42 & .85 & 10.5 & 2.5 \\
\hline R56 & 28 & .00224372 & .00000121 & .00169697 & .00000120 & 28.77 & .86 & $"$ & \\
\hline R59 & 35 & .00825644 & -.00014972 & .00495119 & -.00014919 & 47.97 & .86 & 15 & 1.5 \\
\hline R60 & 39 & .00001325 & -.00000000 & .00001075 & -.00000000 & 40.38 & .91 & 13 & 5.5 \\
\hline R61 & 40 & .00012458 & .00000000 & .00019609 & .00000000 & 21.45 & .91 & 15 & 4 \\
\hline R62 & 45 & .00018122 & -.00000001 & .00031959 & -.00000001 & 21.98 & .91 & 18 & 3.5 \\
\hline R63 & 48 & .00088430 & -.00000000 & .00386011 & -.00000001 & 9.54 & .89 & 20 & 3 \\
\hline R68 & 80 & .00001735 & .00000000 & .00009354 & .00000000 & 13.68 & .92 & 35 & 1 \\
\hline
\end{tabular}


Table V: IS Type, Within Comparisons

Lower bound Upper bound

$\mathbf{S}$

v

of $\Phi_{2} / \Phi_{1}=\mathrm{L}$

1.96551720

1.96551720

1.44827580

1. 72391020

1. 25556860

of $\Phi_{2} / \Phi_{1}=\mathrm{P}$

2.00000000

2.00000000

1.49999990

1.79999990

1. 28571420 (2)

1. 89130490

.50000000

.50000010

2. 30390930

2.64363930

2. 98104600

3.65261530
2. 00000000

2. 00000000

.59340660

.42615020

2.00000000

.52439030

.55555560

2. 33333333

2.66666740

3. 00001610

3. 66668620
Error of<smiles>[LiH]</smiles>

$\mathrm{G}_{2}$

.31913521

.31913521

.35289453

.33148280

. 37094270
.00000056

.00000056

.00000074

.00000062

.00000087
.26366817

.26366817

. 32071417

.28723591

. 34998933
Error of

$$
\mathrm{G}_{2}
$$

B

D

$\begin{array}{lll}.00000028 & 3.29 & .52 \\ .00000028 & 3.48 & .55 \\ .00000053 & 3.08 & .54 \\ .00000037 & 3.33 & .55 \\ .00000071 & 3.06 & .57\end{array}$

.00000028

$.29 \quad .52$

.55

.55

57
.31913521
.31913521
.07081078
.07412205
.05368397
.07211909
.01089548
.00123686
.00016765
.00002149
.00000030$$
26206513
$$

.00000028

.00000028

$-.00013790$

$-.00013751$

$-.00014098$

$-.00013773$

.00000000

.00000128

$-.00000000$

$-.00000001$

.00000000 112.48 .95

$\begin{array}{rl}3.41 & .54 \\ 3.54 & .57 \\ 8.68 & .80 \\ 8.26 & .79 \\ 10.26 & .71 \\ 8.52 & .80 \\ 16.59 & .86 \\ 34.46 & .91 \\ 50.60 & .93 \\ 69.36 & .95 \\ 12.48 & .97\end{array}$

I 
Table* VI: LS Type, Among Comparisons

$\begin{array}{rrrrrrrr}\mathrm{S} & \mathrm{V} & \mathrm{G}_{1} & \text { Error of } \mathrm{G}_{1} & \mathrm{G}_{2} & \text { Error of } \mathrm{G}_{2} & \mathrm{~B} & \mathrm{D} \\ & & & & & & \\ 1 & 9 & 1.87617270 & .00028460 & 3.43970230 & .00028460 & 1.48 & .52 \\ 2 & 9 & 1.87617270 & .00028460 & 3.43970230 & .00028460 & 1.57 & .55 \\ 3 & 9 & 1.46043870 & .00028460 & 2.12674590 & .00028460 & 1.92 & .52 \\ 4 & 9 & 1.68620910 & .00028460 & 2.88645690 & .00028460 & 1.67 & .53 \\ 5 & 9 & 1.29601880 & .00028460 & 1.63940220 & .00028460 & 2.28 & .55 \\ 6 & 9 & & & & & & \\ 7 & 9 & & & & & & \\ 8 & 9 & & & & & & \\ 9 & 9 & & & & & & \\ 10 & 9 & 1.89020710 & .00028460 & 3.43970230 & .00028460 & 1.54 & .54 \\ 11 & 9 & 1.89020700 & .00028460 & 3.43970230 & .00028460 & 1.60 & .57 \\ 12 & 16 & .10180204 & -.00000000 & .06678308 & .00000000 & 14.81 & .75 \\ 13 & 16 & .07346688 & -.00000000 & .03668556 & .00000000 & 19.60 & .72 \\ 14 & 16 & .28204712 & -.00000000 & .52623560 & -.00000001 & 4.80 & .68 \\ 15 & 16 & .09018610 & -.00000000 & .05348033 & .00000000 & 16.46 & .74 \\ 16 & 25 & .01445477 & -.00014358 & .00943603 & -.00014312 & 28.36 & .72 \\ 17 & 36 & .00838014 & .00000004 & .01730837 & .00000004 & 14.26 & .90 \\ 18 & 49 & .00136756 & .00000135 & .00316312 & -.00000137 & 18.40 & .93 \\ 19 & 64 & .00020687 & -.00000000 & .00052993 & -.00000001 & 22.50 & .94 \\ 20 & 100 & .00000388 & .00000000 & .00001188 & .00000000 & 30.61 & .96\end{array}$

*The lower and upper bounds of $\Phi_{2} / \Phi_{1}$ are given in Table $V$. 
Table* VII: Triangular Designs, Within Comparisons

\begin{tabular}{|c|c|c|c|c|c|c|c|c|c|c|c|c|}
\hline $\begin{array}{l}\text { No. } \\
\text { of } \\
\text { design }\end{array}$ & $v$ & $\begin{array}{l}\text { Lower } \\
\text { bound } \\
\text { of } \psi_{3} / \psi_{1}\end{array}$ & $\begin{array}{l}\text { Upper } \\
\text { bound } \\
\text { of } \psi_{3} / \psi_{1}\end{array}$ & $\begin{array}{l}\text { Lower } \\
\text { bound } \\
\text { of } \psi_{2} / \psi_{1}\end{array}$ & $\begin{array}{l}\text { Upper } \\
\text { bound } \\
\text { of } \psi_{2} / \psi_{1}\end{array}$ & $\mathrm{G}_{2}$ or $\mathrm{E}_{2}$ & $\begin{array}{l}\text { Error of } \\
\mathrm{G}_{2}\end{array}$ & $\mathrm{G}_{1}$ or $\mathrm{E}_{1}$ & $\begin{array}{l}\text { Error of } \\
G_{1}\end{array}$ & B & $\mathrm{D}$ & \\
\hline $\mathrm{T} 6$ & 10 & .42857120 & .46938750 & .57142840 & .60204070 & . . 48272526 & -.00000000 & .37314616 & .00011290 & 2.62 & .49 & \\
\hline T7 & 10 & 1.00137230 & 1.02147270 & 1.00102920 & 1.01610450 & .33310462 & .00000000 & .33199972 & .00011336 & 3.75 & .62 & \\
\hline T8 & 10 & .42857150 & .46938780 & .57142860 & .60204090 & .48272516 & -.00000000 & .37314613 & .00011290 & 2.98 & .56 & \\
\hline $\mathrm{T} 12$ & 10 & 1.62068960 & 1.64285710 & 1.82758620 & 1.85714280 & .25731060 & -.00000000 & .27927481 & .00011432 & 3.96 & .55 & \\
\hline T13 & 10 & 1.62068960 & 1.64285710 & 1.82758610 & 1.85714280 & .25731060 & -.00000000 & .27927481 & .00011432 & 4.18 & .58 & \\
\hline $\mathrm{T} 14$ & 10 & 1.28817730 & 1.32142850 & 1.38423640 & 1.42857140 & .29228316 & .00000000 & .30268360 & .00011383 & 3.89 & .59 & N \\
\hline T18 & 10 & 1.63700830 & 1.64286130 & 1.84934440 & 1.85714840 & .25583841 & .00000000 & .27927454 & .00011432 & 4.16 & .58 & 1 \\
\hline T19 & 10 & .42857450 & .43072750 & .57143090 & .57304560 & .48272390 & -.00000000 & .37672160 & .00011286 & 3.00 & .57 & \\
\hline $\mathrm{T} 23$ & 15 & .46666660 & .52647970 & .80000000 & .82242990 & 1.34026490 & & 1.04359710 & & 6.51 & .62 & \\
\hline $\mathrm{T} 24$ & 15 & .46666660 & .52647970 & .80000000 & .82242990 & 1.34026490 & & 1.04359710 & & 6.80 & .65 & \\
\hline T30 & 15 & 1.12550610 & 1.14285720 & 1.33468310 & 1.38095270 & .94547610 & & .92176423 & & 8.57 & .72 & \\
\hline
\end{tabular}

*The accuracy of $\mathrm{E}_{1}$ and $\mathrm{E}_{2}$ is to the sixth decimal place (see Section 8.11). 
Table* VIII: Traingular Designs, Among Comparisons

\begin{tabular}{|c|c|c|c|c|c|c|c|}
\hline $\begin{array}{l}\text { No. of } \\
\text { design }\end{array}$ & $\mathrm{v}$ & $E_{2}$ or $G_{2}$ & Error of $\mathrm{G}_{2}$ & $\mathrm{E}_{1}$ or $\mathrm{G}_{1}$ & Error of $G_{1}$ & B & D \\
\hline T6 & 10 & 1. 19818240 & & 1.05692300 & & 4.96 & .37 \\
\hline $\mathrm{T7}$ & 10 & 99986668 & & .99799656 & & 3.69 & .61 \\
\hline $\mathrm{T} 8$ & 10 & 1. 19818080 & & 1.05692300 & & 5.64 & .43 \\
\hline $\mathrm{T} 12$ & 10 & .87732533 & & .91344069 & & 2.05 & .51 \\
\hline T13 & 10 & .87732533 & & .91344069 & & 2.16 & .53 \\
\hline T14 & 10 & .93602815 & & .95229941 & & 2.68 & .55 \\
\hline T18 & 10 & .87476619 & & .91344014 & & 2.14 & .53 \\
\hline T19 & 10 & 1.19817970 & & 1.06176610 & & 5.95 & .45 \\
\hline T23 & 15 & .22027083 & .00000000 & .18703621 & .00011599 & 8.63 & .38 \\
\hline $\mathrm{T} 24$ & 15 & .22027083 & .00000000 & .18703621 & .00011599 & 9.02 & .39 \\
\hline T30 & 15 & .17512971 & -.00000000 & .17211884 & .00011664 & 6.26 & .61 \\
\hline
\end{tabular}

*The accuracy of $E_{1}$ or $E_{2}$ is to the sixth decimal place (see Section 8.11). 
Table* IX: Cyclic Designs, within Comparisons

\begin{tabular}{|c|c|c|c|c|c|c|c|c|c|}
\hline \multirow{2}{*}{$\begin{array}{l}\text { No. of } \\
\text { design }\end{array}$} & \multirow[b]{2}{*}{$\mathrm{v}$} & $\begin{array}{l}\text { Lower } \\
\text { bound }\end{array}$ & $\begin{array}{l}\text { Upper } \\
\text { bound }\end{array}$ & $\begin{array}{l}\text { Lower } \\
\text { bound }\end{array}$ & $\begin{array}{l}\text { Upper } \\
\text { bound }\end{array}$ & \multirow[b]{2}{*}{$\mathrm{E}_{1}$} & \multirow[b]{2}{*}{$\mathbf{E}_{2}$} & \multirow[b]{2}{*}{ B } & \multirow[b]{2}{*}{ D } \\
\hline & & of $\psi_{3} / \psi_{1}$ & of $\psi_{3} / \psi_{1}$ & of $\psi_{2} / \psi_{1}$ & of $\psi_{2} / \psi_{1}$ & & & & \\
\hline 1 & 13 & .33333333 & .37037010 & .83333333 & .84259260 & 1.06178250 & 1.92264240 & 3.38 & .40 \\
\hline$=2$ & 13 & 1.19143980 & 1.20000000 & 1.76575940 & 1.80000000 & .78509506 & .87702937 & 6.08 & .53 \\
\hline 3 & 13 & 1.06262920 & 1.07142860 & 1.25051680 & 1.28571460 & .90801427 & .95631297 & 6.45 & .65 \\
\hline 4 & 13 & .33333340 & 050 & .83333340 & 270 & 1.06565970 & 1.92264370 & 3.81 & .45 \\
\hline 5 & 17 & 1 & 80 & 1.36 & 380 & .88794416 & .91941955 & 10.38 & .71 \\
\hline 66 & 17 & .53333340 & .5363 & .83333340 & .83439960 & 1.05928760 & 1.43911020 & 7.94 & .65 \\
\hline 27 & 17 & .53333270 & .53539250 & .83333310 & .83406880 & 1.05941500 & 1.43910700 & 7.97 & .65 \\
\hline 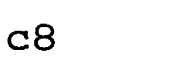 & 29 & .57639040 & .58160980 & .91666700 & .91769380 & 1.02510200 & 1.32158740 & 17.60 & .72 \\
\hline$c 9$ & 29 & .2 & 30 & $.85^{\circ}$ &.$\varepsilon$ & 443640 & 1.69198110 & 14.04 & .59 \\
\hline 10 & 37 & .70634920 & $\$ 10$ & .88517270 & 770 & 1.02648960 & 1.17377540 & 26.82 & .83 \\
\hline
\end{tabular}

*The accuracy of $\mathrm{E}_{1}$ or $\mathrm{E}_{2}$ is to the sixth decimal place (see Section 8.11). 
Table* X: Cyclic Designs, Among Comparisons

\begin{tabular}{|c|c|c|c|c|c|}
\hline $\begin{array}{l}\text { No. of } \\
\text { design }\end{array}$ & $\mathbf{v}$ & $E_{1}$ & $\mathrm{E}_{2}$ & B & D \\
\hline cl & 13 & 1.04573670 & 1.61058820 & 4.72 & .18 \\
\hline$c 2$ & 13 & .83119494 & .90590690 & 3.46 & .38 \\
\hline c3 & 13 & .92964747 & .96708089 & 5.08 & .56 \\
\hline c4 & 13 & 1.04856730 & 1.61059310 & 5.37 & .21 \\
\hline c5 & 17 & .90855906 & .93483468 & 7.52 & .59 \\
\hline$c 6$ & 17 & 1.04693670 & 1.33285940 & 10.16 & .44 \\
\hline c7 & 17 & 1.04703650 & 1.33286350 & 10.19 & .44 \\
\hline$c 8$ & 29 & 1.02190010 & 1.27396560 & 19.83 & .47 \\
\hline c9 & 29 & 1.03868120 & 1.57294470 & 17.51 & .20 \\
\hline $\mathrm{cl0}$ & 37 & 1.02348070 & 1.15243270 & 30.26 & .66 \\
\hline
\end{tabular}

*The accuracy of $\mathrm{E}_{1}$ or $\mathrm{E}_{2}$ is to the sixth decimal place (see Section 8.11). 
Table XI: The $\rho_{i}$ 's for Triangular Designs

No. of

design

T6

T7

T8

T12

T13

T14

T18

T19

T23

T24

T30

$$
\rho_{i}=0, i=1,2, \ldots, 7 ; \quad \rho_{8}=\frac{12}{7}, \quad \rho_{9}=\frac{9}{7}
$$

"1

"I

11

II

II

"1

"

"

"

II

"I

11

"

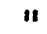

II

II

I

II

II

II
I

$$
\rho_{i}=0, i=1,2, \ldots, 11 ; \quad \rho_{12}=\frac{8}{3}, \quad \rho_{13}=1, \rho_{14}=\frac{7}{3}
$$

II

"

II

II

II

1

II

"

II 
No. of

design

CI

$\rho_{i}=0, \quad i=1,2, \ldots, 8 ; \quad \rho_{9}=2, \rho_{10}=2, \rho_{11}=\frac{3}{2}, \rho_{12}=\frac{1}{2}$

$\mathrm{c} 2$

c3

c4

c5

c6

C7

c8 $\rho_{i}=0, i=1,2, \ldots, 20 ; \rho_{21}=\frac{5}{2}, \rho_{22}=\frac{11}{6}, \rho_{23}=\frac{61}{24}, \rho_{24}=\frac{97}{40}, \rho_{25}=\frac{11}{5}, \rho_{26}=1, \rho_{27}=1$,

Table XII: The $p_{i}$ 's for Cyclic Designs

1

$$
"
$$

"

"

"

"I

"

II

" 1

$"$

4

$"$

11

11

$$
\rho_{i}=0, i=1,2, \ldots, 11 ; \rho_{12}=\frac{7}{3}, \rho_{13}=\frac{7}{3}, \rho_{14}=\frac{5}{6}, \rho_{15}=\frac{43}{30}, \rho_{16}=\frac{16}{15}
$$

6

"

II

"

"1

"

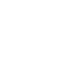

c9
"

$"$

11

"

$$
\rho_{28}=\frac{1}{2}
$$

C10 $\rho_{i}=0, i=1,2, \ldots, 27 ; \rho_{28}=\frac{5}{2}, \rho_{29}=\frac{5}{3}, \rho_{30}=\frac{47}{23}, \rho_{31}=\frac{91}{40}, \rho_{32}=\frac{11}{5}, \rho_{33}=\frac{37}{14}$,

$$
\rho_{34}=\frac{243}{112}, \quad \rho_{35}=\frac{8}{5}, \quad \rho_{36}=\frac{3059}{2960}
$$




\section{ABSTRACT}

We know that the best linear combination of the intraand inter-block estimates is

Intra-estimatexInter-variance+Inter-estimatexIntra-variance Intra-variance + Inter-variance

however, this combined estimate is merely theoretical, since we do not know in practice the exact inter- and intravariances. A reasonable solution is to use a random weight which can be computed from the data of our experiment, but so far there has been no practical solution without severe restrictions on the size of the experiment, and no solution at all for a clear answer to the question of how much we recovered. In fact, the experimenter applying the methodology available to him now, cannot be sure that he is really improving the accuracy of his estimation.

This research has achieved the following:

1. A new method of combining two independent estimates has been developed. This method has its use in incomplete block designs, in similar experiments, and in randomized block designs with heterogeneous variances. The improvement introduced by this method is very satisfactory, compared with the utmost possible theoretical improvement. 
2. A procedure for recovering the inter-block information in B.I.B. designs was given, which is applicable in experiments of as small as $t=4$.

3. It has been proven that the practical utilization of inter-block information is possible in any P.B.I.B. with seven treatments or more.

4. A general procedure for recovering the inter-block information in P.B.I.B.'s with two associate classes was given.

5. An inter-block analysis of singular and semi-regular group divisible designs was discussed, which makes a partial utilization of the inter-information possible.

In general, this work has two merits:

1. It makes possible the utilization of the interblock information in small and moderate size experiments.

2. As a ratio of the utmost possible theoretical recovery (by combining linearly), either exactly or a lower bound of the ratio of recovery is always computable.

Tables which enable the experimenter to use the procedures described in this dissertation were given. The ratios of recovery listed in these tables show that the new method gives good results where the old method is not applicable, 
and when the old method starts, hopefully, to be valid, the ratio of recovery achieved by the new method starts to approach the theoretical value that can be achieved, assuming the intra- and inter-variance are known. 